\title{
Towards the assessment of a residential electric storage system: analysis of Canadian residential electricity use and the development of a lithium-ion battery model
}

\author{
by \\ Neil Saldanha, B.Sc., Mechanical Engineering \\ Queen's University
}

\begin{abstract}
A thesis submitted to the
Faculty of Graduate and Postdoctoral Affairs

in partial fulfillment of the requirements for the degree of
\end{abstract}

Master of Applied Science in Mechanical Engineering

Ottawa-Carleton Institute for Mechanical and Aerospace Engineering

Department of Mechanical and Aerospace Engineering

Carleton University

Ottawa, Ontario

August, 2010

(C)Copyright

Neil Saldanha, 2010 


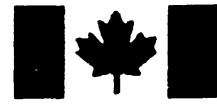

\section{Library and Archives \\ Canada \\ Published Heritage Branch}

395 Wellington Street

Ottawa ON K1A 0N4

Canada
Bibliothèque et

Archives Canada

Direction du

Patrimoine de l'édition

395, rue Wellington

Ottawa ON K1A ON4

Canada
Your file Votre référence

ISBN: 978-0-494-71561-1

Our file Notre reférence

ISBN: 978-0-494-71561-1
NOTICE:

The author has granted a nonexclusive license allowing Library and Archives Canada to reproduce, publish, archive, preserve, conserve, communicate to the public by telecommunication or on the Internet, loan, distribute and sell theses worldwide, for commercial or noncommercial purposes, in microform, paper, electronic and/or any other formats.

The author retains copyright ownership and moral rights in this thesis. Neither the thesis nor substantial extracts from it may be printed or otherwise reproduced without the author's permission.
AVIS:

L'auteur a accordé une licence non exclusive permettant à la Bibliothèque et Archives Canada de reproduire, publier, archiver, sauvegarder, conserver, transmettre au public par télécommunication ou par l'Internet, prêter, distribuer et vendre des thèses partout dans le monde, à des fins commerciales ou autres, sur support microforme, papier, électronique et/ou autres formats.

L'auteur conserve la propriété du droit d'auteur et des droits moraux qui protège cette thèse. $\mathrm{Ni}$ la thèse ni des extraits substantiels de celle-ci ne doivent être imprimés ou autrement reproduits sans son autorisation.
In compliance with the Canadian Privacy Act some supporting forms may have been removed from this thesis.

While these forms may be included in the document page count, their removal does not represent any loss of content from the thesis.
Conformément à la loi canadienne sur la protection de la vie privée, quelques formulaires secondaires ont été enlevés de cette thèse.

Bien que ces formulaires aient inclus dans la pagination, il n'y aura aucun contenu manquant. 
The undersigned hereby recommends to the

Faculty of Graduate and Postdoctoral Affairs acceptance of the thesis

Towards the assessment of a residential electric storage system: analysis of Canadian residential electricity use and the development of a lithium-ion battery model

submitted by Neil Saldanha, B.Sc., Mechanical Engineering Queen's University

in partial fulfillment of the requirements for the degree of Master of Applied Science in Mechanical Engineering 
Professor Ian Beausoleil-Morrison, Thesis Supervisor

Professor Cynthia Cruickshank

Professor Steven McGarry

Professor Atef Fahim

Dr. Ken Darcovich

Professor Metin Yaras, Chair,

Department of Mechanical and Aerospace Engineering

Ottawa-Carleton Institute for Mechanical and Aerospace Engineering

Department of Mechanical and Aerospace Engineering

Carleton University

August, 2010 


\section{Abstract}

Peak electricity demand from residential houses leads to increased greenhouse gas emissions from inefficient electricity production. The coincidental use of household appliances and lighting, known as non-HVAC loads, and air-conditioners creates periods of increased electricity demand on utility providers in Ontario. This leads to electricity production from fuels such as coal that produce excessive greenhouse gas emissions.

To assess the potential of a micro-cogeneration device coupled with lithium-ion battery electricity storage to reduce peak electricity demand using building simulation, residential electricity use of Canadian houses must be accurately represented. Thus, electricity use from twelve houses in the Ottawa, Ontario area was collected over a one year period, beginning in the summer of 2009. The project measured and analyzed non-HVAC and space cooling electricity use at one-minute intervals. The daily non-HVAC electricity profiles measured in this study show more variation and higher occurrences of peak loads compared to previously developed synthetically generated profiles. Both non-HVAC and space cooling profiles show large variations in electricity consumption between households. The relationship between daily space cooling electricity consumption and outdoor temperature is shown.

A lithium-ion battery model was then developed in the building simulation program ESP-r. The model accounts for changes in performance due to varying temperature and current, and addresses long-term degradation over a battery's life cycle.

The model was calibrated and validated using simulated data from the National Research Council's Institute for Chemical Process and Environmental Technology. The functionality of the lithium-ion model simulated in a household and coupled with a Stirling engine micro-cogeneration model is demonstrated.

Following the implementation of an optimized controller, a lithium-ion battery simulated with a micro-cogeneration device can be sized and fully assessed. The research in this document provides the foundation for this assessment. 
To my family for keeping me grounded, and my friends for keeping me up late... 


\section{Acknowledgments}

First and foremost I would like to acknowledge my supervisor, Dr. Ian BeausoleilMorrison. Not only would this work had not been possible without his academic guidance, but his personal mentorship and approach to life will be something that I will never forget.

I would like to acknowledge Dr. Ken Darcovich, Dr. Isobel Davidson and Dr. Eduardo Henquin at the National Research Council's Institute for Chemical Process and Environmental Technology. Their support and guidance helped me to branch into a new area of science.

Special thanks also goes to Dave Kuhnle and Steve Kuhnle of Nepean Electric. Not only did they help me in getting this project off its feet, but their expertise and patience went beyond the call of professionalism.

Thanks as well to Dr. Guy Newsham at the National Research Council's Institute for Research in Construction. Their support and funding allowed the results from this project to exceed further.

Lastly, a very special thank you to my colleagues and friends in The Sustainable Building Energy Systems Laboratory at Carleton University. I came to this school not knowing what to expect, but your support throughout this project made it, and I, succeed. 


\section{Table of Contents}

Abstract $\quad$ iv

Acknowledgments vi vi

Table of Contents vii

List of Tables $\quad$ ix

List of Figures $\quad$ x

Nomenclature $\quad$ xv

1 Introduction and literature review 1

1.1 Introduction . . . . . . . . . . . . . . . . 1

1.2 Litcrature revicw . . . . . . . . . . . . . . . . . 2

1.2.1 Canadian residential energy use and the electric grid . . . . 2

1.2 .2 Micro-cogeneration . . . . . . . . . . . . . . 5

1.2.3 Electricity storage and lithium ion batteries . . . . . . . 6

$1.2 .4 \quad$ RES modelling . . . . . . . . . . . . . . . 8

1.2 .5 Residential load monitoring . . . . . . . . . . . 9

1.3 Research objectives . . . . . . . . . . . . . . . . . . . . 10

1.4 Thesis outline . . . . . . . . . . . . . . . . . 11

2 ESP-r simulation methods $\quad 12$

2.1 The ESP-r thermal, air flow and plant networks . . . . . . . . . . 12

2.2 Electrical power flow network in ESP-r . . . . . . . . . . 17

3 Non-HVAC and Space Cooling Electric Loads 21

3.1 Previous work and synthetically gencrated profiles . . . . . . . . . 21 
3.2 Electrical measurement project . . . . . . . . . . . . . . 24

3.2.1 Measurement methods and data analysis . . . . . . . . 26

3.3 Non-HVAC data results . . . . . . . . . . . . . . . . 32

3.4 Space cooling data analysis . . . . . . . . . . . . . . . . 42

4 Lithium-ion battery model $\quad 47$

4.1 Lithium-ion battery chemistry . . . . . . . . . . . . . . . . . 47

4.2 Lithium-ion battery modelling . . . . . . . . . . . . . . 53

4.3 Li-ion modelling approach for present work . . . . . . . . . . 56

4.4 Implementation in ESP-r battery subroutine . . . . . . . . . . . 59

5 Calibration of the Battery Model $\quad 65$

51 Calibrated source data . . . . . . . . . . . . . . 65

5.2 Calibration parameters . . . . . . . . . . . . 67

5.2 .1 Geometric paraneters . . . . . . . . . . . . 68

5.2 .2 Current and temperature parameters . . . . . . . . . 74

6 Demonstration of new modelling capabilities $\quad 84$

6.1 Li-ion battery model debugging . . . . . . . . . . . . . . . 84

6.2 Validation with simulated data from ICPET . . . . . . . . 85

6.3 Demonstration of integrated system . . . . . . . . . . . 88

6.3 .1 ESP-r model description . . . . . . . . . . . . . . . 88

6.3 .2 Sinulation results . . . . . . . . . . . . . . . . 90

7 Conclusions \& Future Work $\quad 95$

7.1 Conclusions . . . . . . . . . . . . . . . . . . 95

7.2 Future Work: Non-HVAC and space cooling . . . . . . . . . . . . 97

7.3 Future Work: Li-ion battery model . . . . . . . . . . . . . . 100

$\begin{array}{ll}\text { List of References } & 105\end{array}$

Appendix A Example of calibration data provided from NRC-ICPET $\left(0.01 \mathrm{~A} \cdot \mathrm{m}^{-2}\right.$ and $\left.25^{\circ} \mathrm{C}\right) \quad 112$

Appendix B Calibration parameters for the Li-ion battery model 114 


\section{List of Tables}

3.1 Annual electric consumption of the participating households in $k W h$. $y r^{-1}$. Note that bias exists due to the lack of a complete year of collected data. . . . . . . . . . . . . . .

3.2 Watt-hours per pulse resolution in logging equipment in the measureinent project. . . . . . . . . . . . . . 27

3.3 Bias errors of measuring devices. . . . . . . . . . . . . . 28

3.4 95th percentile non-HVAC electrical power draw of the households in the measurement project sorted by annual energy consumption level at one minute intervals. . . . . . . . . . . . . . . . 34

3.5 95th percentile non-HVAC electrical power draw of the synthetically generated profiles sorted by annual energy consumption level. . . . .

5.1 List of data sets provided by NRC-ICPET. 16 data sets of discharge curves were provided, each one at either constant current or constant temperature. . . . . . . . . . . . . . 66

5.2 Internal resistance coefficients used for the model calibration. . . . . 78

5.3 Internal resistance coefficients used for model calibration. . . . . . 80

$5.4 \Delta V(T)$ values to calibrate li-ion battery model. . . . . . . . . 83

6.1 Current and temperature statistics for annual simulation. . . . . . 92 


\section{List of Figures}

1.1 Simulation of electricity used for space cooling in a typical Canadian dwelling in Ottawa, Ontario on June 7th, 2008. . . . . . . . . 3

1.2 Market electricity demand and supply by generation source in Ontario on June 7th. 2008. Note: "Other" indicates gas fired and wood waste sources. ........................ 4

1.3 Schematic of lithium ion battery. . . . . . . . . . . . . 7

1.4 Schematic of Li-ion micro-cogeneration model. . . . . . . . . . . . . . 9

2.1 Energy flow paths and energy generation at an air point node in ESP-r. 14

2.2 Energy flow paths and energy generation at construction nodes in ESP-r. 14

2.3 Schematic of example HVAC system in ESP-r. . . . . . . . . . . . 16

2.4 Matrix equations for thermal domain at $\imath^{\text {th }}$ node with plant injection. 18

2.5 Example of elcctrical network schematic in ESP-r. . . . . . . . . . . 19

3.1 Comparison of typical electricity profile taken at one minute intervals and averaged over one hour intervals. . . . . . . . . . . . 23

3.2 Measuring devices installed at the electric panel of a participant in the electric measurement project. . . . . . . . . . . . .

3.3 Bias crror as a function of average power drawn by the entire houschold over one minute. Note that this is for a $240 \mathrm{~V}$ circuit with a $50 \mathrm{ACT}$ attached, and is assumed to be working within an acceptable current range and with a phase angle of zero. . . . . . . . . . . . . .

3.4 95th percentile illustration from a cumulative histogram from the data

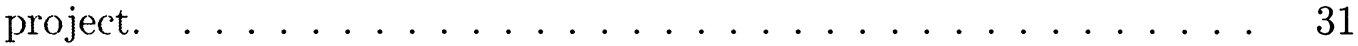

3.5 Example of data "smoothing" as a result of too coarsc of a measuring resolution for a one minute logging interval. . . . . . . . . . . . 33

3.6 Household H12's electricity use at one minute intervals on Aug. 11,

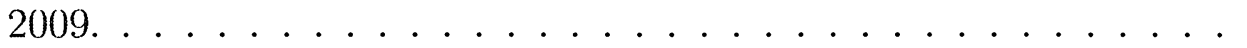


3.7 Houschold H10's electricity use at one minute intervals on Aug. 11,

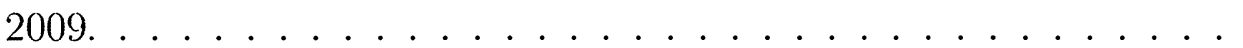

3.8 Daily average non-HVAC electricity power draw of all the households in the project, separated by their encrgy category, by season in $k W$ at one minute intervals. . . . . . . . . . . . . .

3.9 Household H1's average non-HVAC household daily electricity draw plotted with two randomly selected days. . . . . . . . . . . . .

3.10 Daily household non-HVAC electricity power draw standard deviation using all the collected clata for each house, separated by energy cate-

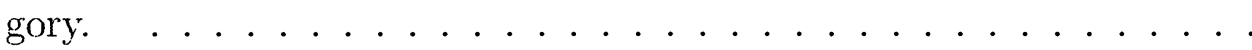

3.11 Probability distribution of household H6's summer weekday non-HVAC electrical power draw between 0:00 and 0:05 in the morning. . . . . .

3.12 Comparison of normalized histograms of measured non-HVAC electric power draws of three medium houses in the measurement project with synthetically generated profiles over the year. The three bands from left to right on the top represent $\mathrm{H} 5, \mathrm{H} 6$ and $\mathrm{H} 7$ medium energy households from the project compared with the three bands from left to right on the bottom representing the three medium energy synthetically gencrated annual profiles. . . . . . . . . . . . .

3.13 Comparison of normalized histograms of measured non-HVAC electric power draws of three medium houses in the measurement project with synthetically generated profiles at higher fractions of the household 95 th percentile. The three bands from left to right on the top represent H5, H6 and $\mathrm{H} 7$ medium energy houscholds from the project compared with the three bands from left to right on the bottom representing the three medium energy synthetically generated annual profiles. . . . . .

3.14 Example of air-conditioning variation between houscholds on August 17 th, 2009 with a mean outdoor temperature of $25.6^{\circ} \mathrm{C} \ldots \ldots \ldots$

3.15 Example of monthly electricity consumption of one of the houses in

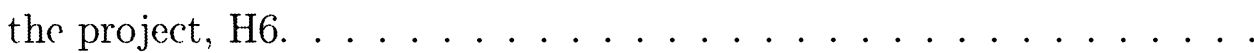

3.16 Household air-conditioning unit daily electrical consumption as a function of maximum and mean daily temperature. Note: Circulation fan electricity consumption is not included in this plot. . . . . . . . . 
4.1 Open circuit voltage (OCV) as a function of DOD (1-SOC) for a

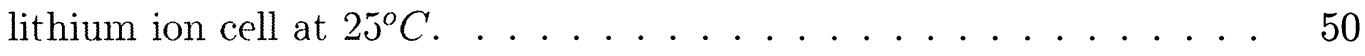

4.2 Voltage as a function of DOD (1-SOC) for a lithium ion cell with increasing current. . . . . . . . . . . . . .

4.3 Voltage as a function of DOD (1-S()C) for a lithium ion cell with increasing tenperature. . . . . . . . . . . . . . . 52

4.4 Diagram of remaining battery capacity as a function of cycle life. . . 53

4.5 RC network schematic used in lumped capacitance modelling of li-ion

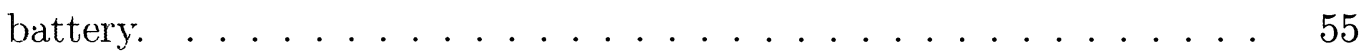

4.6 Transformation of voltage as a function of DOD curve due to an imposed current. . . . . . . . . . . . . . . . 57

4.7 Transformation of voltage as a function of DOD curve due to a change in temperature. . . . . . . . . . . . . . . 58

4.8 Battery management system used in li-ion battery subroutine. . . . . 64

5.1 Schematic of heat loss to surroundings from battery pack. . . . . . . 70

52 Schematic of cell resistors in series and parallel making up an overall battery resistance. . . . . . . . . . . . . . . . . 72

5.3 OCV polynomial fit using a least squares method to original simulated data from ICPET. . . . . . . . . . . . . . . . . . 74

5.4 Voltage as a function of DOD over a range of currents. . . . . . . 75

5.5 Internal resistance as a function of DOD over a range of currents. . . 76

5.6 Plot of polynomial fit of original data from ICPET, shown as dashed lines, compared with an OCV curve scaled by an internal resistance curve. shown as "x"s. . . . . . . . . . . . . . . . . . . . 77

5.7 Voltage as a function of DOD over a range of currents. . . . . . . 79

5.8 Change in voltage as a function of DOD due to cell temperature with respect to the $25^{\circ} \mathrm{C}$ reference curve. . . . . . . . . . . 81

5.9 Change in voltage as a function of DOD due to cell temperature with respect to the $25^{\circ} \mathrm{C}$ reference curve. . . . . . . . . . . 82

6.1 Example of coding crror discovered during debugging. . . . . . . . 85

62 Comparison of li-ion simulation run in ESP-r with simulated data from ICPET . . . . . . . . . . . . . . . . .

6.3 Comparison of same simulation in ESP-r with different time-steps to illustrate sensitivity. . . . . . . . . . . . . . . . . 
6.4 Plant system schematic for application demonstration. . . . . . . . .

6.5 Household electricity demand, battery load and battery DOD over two days of simulation beginning August 4th, 2008. By convention, a positive battery load indicates a battery discharge and a negative indicates

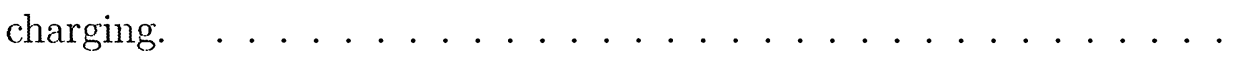

6.6 Battery voltage. current and temperature over two days of simulation beginning August 4th, 2008. By convention, a positive current indicates a battery discharge and a negative indicates charging. . . . . . 91

6.7 Total monthly heat dump from the SE to the ambient surroundings. 93

6.8 Simulation comparison with and without use of li-ion battery. The total monthly electric grid imports and exports are shown for both scenarios. . . . . . . . . . . . . . . . .

7.1 Auto-corrclation as a function of lag $\mathrm{k}$ time-steps during on-peak hours for a randomly selected day of a randomly selected household in the

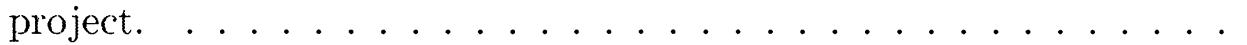

7.2 Auto-corrclation as a function of lag $\mathrm{k}$ timesteps during off-peak hours for a randomly selected day of a randomly selected household in the project. . . . . . . . . . . . . . . . . . 99

7.3 Example of custom controller flowrchart for RES. . . . . . . . . . . 104 


\section{Nomenclature}

\begin{tabular}{|c|c|c|}
\hline$D O D$ & Depth of discharge & $(-)$ \\
\hline$S O C$ & State of charge & $(-)$ \\
\hline$O C V$ & Open circuit voltage & $V$ \\
\hline$L i^{+}$ & Percentage of lithium ion content & $(-)$ \\
\hline$m$ & Mass & $k g$ \\
\hline$\dot{m}$ & Mass flow rate & $k g \cdot s^{-1}$ \\
\hline$C_{p}$ & Specific heat & $J \cdot k g^{-1} \cdot K^{-1}$ \\
\hline$T$ & Battery temperature & ${ }^{\circ} \mathrm{C}$ \\
\hline$t$ & Time & $s$ \\
\hline$\Delta t$ & Time interval & $s$ \\
\hline$S A$ & Surface area & $m^{2}$ \\
\hline$A$ & Area & $m^{2}$ \\
\hline$I$ & Current & $I$ \\
\hline$V$ & Voltage & $V$ \\
\hline$R$ & Resistance & $\Omega$ \\
\hline$\widetilde{I}$ & Phasor current & $I$ \\
\hline$\widetilde{V}$ & Phasor voltage & $V$ \\
\hline$\widetilde{Z}$ & Complex impedance & $\Omega$ \\
\hline$h$ & Heat transfer coefficient & $W \cdot m^{-2} \cdot{ }^{\circ} C^{-1}$ \\
\hline$L$ & Characteristic vertical length & $m$ \\
\hline
\end{tabular}




\begin{tabular}{|c|c|c|}
\hline$n$ & Number of cells & $(-)$ \\
\hline Cap & Battery capacity & $W \cdot h$ \\
\hline$\sigma$ & Stefan-Boltzmann constant & $W \cdot m^{-2} \cdot K^{-4}$ \\
\hline$\epsilon$ & Emissivity & $(-)$ \\
\hline$S_{D}$ & Self discharge current & $A$ \\
\hline$k_{o}$ & Self discharge constant & $A$ \\
\hline$E_{a s}$ & Activation energy of the battery & $k J \cdot k m o l^{-1}$ \\
\hline$\alpha$ & Capacity factor to account for battery current & $(-)$ \\
\hline$\beta$ & Capacity factor to account for battery temperature & $(-)$ \\
\hline$\Delta V(T)$ & Voltage change to account for battery temperature & $V$ \\
\hline$\Delta P$ & Pressure gradient & $k P a$ \\
\hline$\dot{q}$ & Rate of energy with respect to time & $W$ \\
\hline$R_{g}$ & Gas constant & $k J \cdot k m o l^{-1} \cdot K^{-1}$ \\
\hline$\vec{D}$ & Proportionality diffusion coefficient & $m^{2} \cdot s^{-1}$ \\
\hline$C_{A}$ & Mass concentration & $k g \cdot m^{-3}$ \\
\hline$A C F$ & Auto-correlation factor & $(-)$ \\
\hline$k$ & Auto-correlation lag & $\min$ \\
\hline$x$ & Statistical variable & $(-)$ \\
\hline$x$ & Statistical variable & $(-)$ \\
\hline$x$ & Statistical variable & $(-)$ \\
\hline$x$ & Statistical variable & $(-)$ \\
\hline RES & Residential electric storage & \\
\hline TOU & Time of use & \\
\hline DHW & Domestic hot water & \\
\hline BMS & Battery management system & \\
\hline
\end{tabular}




\section{Chapter 1}

\section{Introduction and literature review}

\subsection{Introduction}

Peak electricity demand from residential houses leads to increased greenhouse gas (GHG) emissions from inefficient electricity production. The electricity used for household appliances and lighting is known as non-heating, non-ventilation and non-air-conditioning (non-HVAC) electrical loads. During summer months, airconditioners use electricity to cool households. The coincidence of non-HVAC and space cooling electricity use creates periods of peak electricity demand on utility providers in Ontario. Peak electricity demand leads to electricity production from fuels such as coal that produce excessive GHG emissions, compared to base demand sources such as hydro and nuclear.

Micro-cogeneration devices have been assessed for reducing peak household electric demand using building simulation software. The results from this assessment were less than ideal [1]. It was found that these devices lack the transient production capabilities to satisfy highly varying household electric demands.

The addition of electric storage may improve a micro-cogeneration device's ability to reduce peak electric demand by improving transient performance. Lithium-ion (li-ion) chemistry is becoming the leader in electric storage batteries for its suitable characteristics. Building simulation can assess a micro-cogeneration device coupled with a li-ion battery to reduce peak demand in a Canadian residential context.

To do this, a li-ion battery model would need to be developed and coupled to an existing micro-cogeneration model in a building simulation. The inputs to such a simulation need to be as accurate as possible for the results to be credible, similar to any computer simulation. One of these inputs are household electricity use profiles. 
At present, accurate profiles representing non-HVAC and space cooling electricity use, at a fine time resolution, for a wide spectrum of Canadian households is lacking. Representing these profiles is a non-trivial task and yet necessary for accurate building simulations. A better understanding of household electricity use from acquired data would improve the accuracy of a simulation-based assessment of a micro-cogeneration device coupled with a li-ion battery.

The following reviews in further detail the points discussed above. The end of this chapter states the objectives of this research and the outline to achieve them.

\subsection{Literature review}

\subsubsection{Canadian residential energy use and the electric grid}

Canada's ratification of the Kyoto Protocol seeks to reduce its GHG emissions by $6 \%$ relative to 1990 levels. Total end use GHG emissions have increased 18.5\% from 1990 to 2006 [2]. The following analysis of Canadian residential energy usage illustrates inefficiencies in power production and consumption.

Canadian residential energy use accounted for $16.3 \%$ of the overall secondary energy use by sector in 2007. Electricity accounted for $38.5 \%$ of this energy by source [2]. Residential use of electricity has grown $19.2 \%$ from 1990 to 2007 [2]. This indicates that residential electricity use is a significant portion of the total residential energy use, which is a significant portion of Canada's overall energy use, and is steadily increasing. A daily electric load profile is a plot of the power drawn by a building over the course of a day. Typical residential load profiles have small base loads with short periods of high demand due to the use of space cooling air-conditioners and other household appliances [1].

The occurrence of electric-powered air-conditioning units in residential homes has also increased in recent years. Between 1990 and 2007, the stock of central airconditioning units and room air-conditioning units in Canadian households grew by nearly $180 \%$ and $129 \%$, respectively [2]. Almost $45 \%$ of Canadian households had some type of air-conditioning equipment in 2003 and the province of Ontario had the highest penetration rate with $74 \%$ of households [3]. During the midday hours of the summer months, air-conditioners are operated by many households, creating an increase of electricity demand on the power supply grid.

A report for the Ontario Power Authority noted that air-conditioners had a high 
coincidence with grid peak and a low annual load factor [4]. An annual load factor is the average power draw of a device divided by its peak power draw. This report indicates that while energy use for space cooling does not account for a significant portion of the overall residential energy use, $1.93 \%$ by total end use in 2007 [2], it does lead to periods of peak electricity demand on the utility grid. A simulation of the electricity drawn by an air-conditioner in a typical Canadian dwelling in Ottawa, Ontario on a hot summer day, June 7th, 2008, is shown in figure 1.1. The outdoor temperature reached $31^{\circ} \mathrm{C}$ during the midday [5] and electricity was used by the air-conditioner to maintain the house at $22^{\circ} \mathrm{C}$.

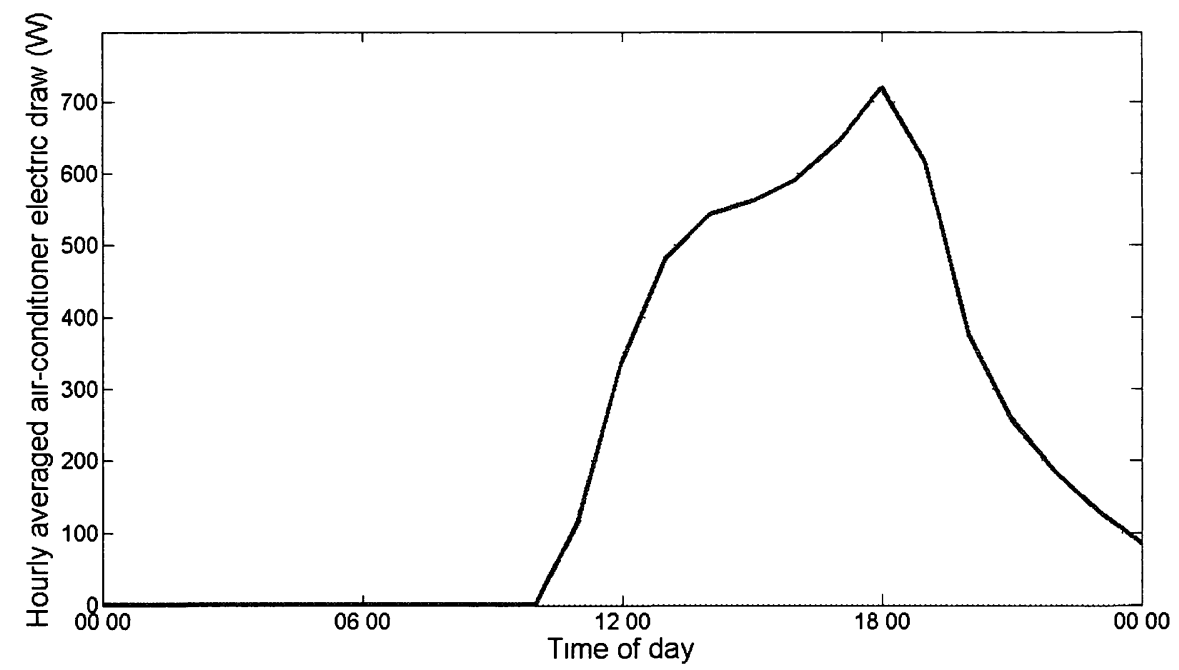

Figure 1.1: Simulation of electricity used for space cooling in a typical Canadian dwelling in Ottawa, Ontario on June 7th, 2008.

In some regions such as Ontario, peak electricity demand is often supplied by generation from fuels with high global warming potentials (GWPs) such as coal, or costly fuels such as natural gas. This is illustrated in figure 1.2 with data collected from the Independent Electric Systems Operator (IESO) [6]. The figure shows the overall market electricity demand and the supply by generation source in Ontario on June 7th, 2008. It should be noted that "other" in the figure indicates gas fired and wood waste sources.

Figure 1.2 shows that electricity generated from coal and gas are two major demand followers and contributors of GHG emissions. In 2007, coal accounted for $76 \%$ 


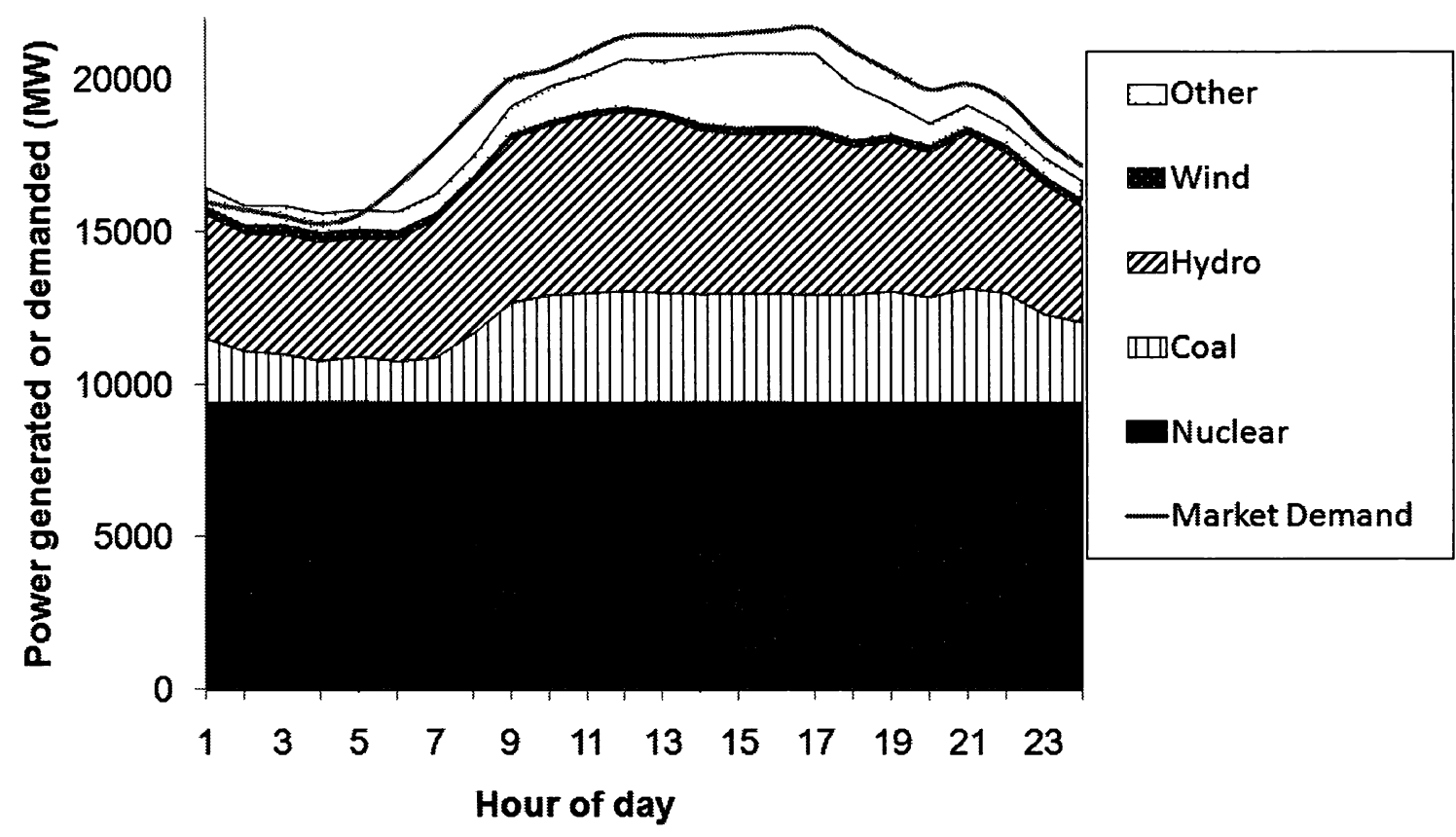

Figure 1.2: Market electricity demand and supply by generation source in Ontario on June 7th, 2008 (data sourced from IESO [6]). Note: "Other" indicates gas fired and wood waste sources.

of all fossil fuels used to generate electricity in Canada [7]. Residential space cooling and appliance usage are significant causes of the peak electric demands that resort to the use of these fuels. Note also that the electricity used for space cooling simulated in figure 1.1 follows the market electricity demand and supply of the same day in figure 1.2. Increases of up to $200 \%$ in transmission losses during peak demand [8] compared to base level demand result in further network inefficiencies. Strategies to reduce residential peak demand need to be explored.

One strategy to reduce peak electricity is demand side management (DSM). DSM attempts to shift non-essential appliance activities, such as clothes washing and drying, from on-peak periods to off-peak periods. Ontario intends to implement a time of use (TOU) program as a form of DSM. When implemented province-wide, occupants will pay more for electricity during on-peak hours than during off-peak hours.

A pilot of the TOU program in Ontario showed an average household utility bill savings of $3 \%$ on the program, compared to the two-tiered pricing scheme currently in place [9]. These savings indicate that users responded to financial incentives and 
shifted a portion of their demand to off-peak times. The only month to show an average household utility bill increase was August. It could be argued that this increase was from air-conditioning use during on-peak times that could not be shifted to off-peak times. This increase shows a flaw in DSM strategies. Appliances such as ovens that cannot be shifted to off-peak times and air-conditioners that are only effective when the outside temperature is high, coincidental with on-peak periods, cannot be influenced by DSM. If the electricity consumed by air-conditioners and appliances that are required during on-peak times could be produced off-peak, stored, and used later, the objective of DSM could be realized. A study by Shaw [10] of loadshifting in the UK showed modest network savings of $0.02 \%$. This study, however, only explored savings in transmission losses during peak periods by theoretically shifting a portion of the on-peak loads to off-peak.

In order to reduce GHG emissions from peak electricity demand at the residential level, other strategies of power production and transmission need to be explored. Micro-cogeneration and electric storage are potential solutions.

\subsubsection{Micro-cogeneration}

Micro-cogeneration devices are showing promising developments for electricity generation at the residential level. These devices are generally either internal combustion engines, solid oxide fuel cells, proton exchange membrane fuel cells, or Stirling engines (SEs). They typically generate less than $10-15 \mathrm{~kW}$ of electricity and are located within the household. Micro-cogeneration devices may defer demand from the utility grid.

When producing electricity alone, micro-cogeneration devices yield poor efficiencies, as low as $5 \%$ in net AC power with reference to the source fuel's lower heating value [1]. However, by using the thermal energy lost in the electrical conversion process for domestic hot water (DHW) and space heating within the home, known as cogeneration, the device's efficiency improves. If done effectively, the efficiency of these devices may be improved from $30-35 \%$ to over $80 \%$, referenced to the source fuel's higher heating value [11].

Micro-cogeneration devices are being implemented worldwide. Although relatively new, SE micro-cogeneration units in France, in 2003, yielded a primary energy savings of $13 \%$. Similar technologies in the UK have yielded primary energy and emissions savings of $28 \%$ [11]. 
The potential of reducing residential electric demand using micro-cogeneration devices was assessed using whole-building computer simulation software. This assessment was Annex 42 of the International Energy Agency's Programme on Energy Conservation in Building and Community Systems (IEA/ECBCS) [1]. It concluded that engine start-up time, high operating temperatures, large thermal inertia, and internal controls preventing high thermal stresses, resulted in poor response to transient electrical loading. Micro-cogeneration transient performance needs improvement to be applied at the residential level, where electricity demand fluctuates rapidly.

A micro-cogeneration device connected to a utility grid can use the grid as an infinite storage source. When the device is generating less electricity than is being demanded from the household, it can import electricity from the grid to make up the difference. The device can also export excess electricity to the grid when its production exceeds the household demand. The excess electricity produced by a few residential cogeneration units can be easily re-distributed through the network. However, for a high penetration rate of micro-cogeneration devices in households, the grid can no longer be used as an infinite storage source. Consequently, storage techniques within the household need to be explored, defined here as residential electric storage (RES).

\subsubsection{Electricity storage and lithium ion batteries}

Many benefits may be achieved from the addition of electric storage. Ibrahim et al. argue that storage could alleviate periods of peak demand, relieve grid congestion, improve infrastructure and prevent power outages on catastrophic scales such as blackouts [12].

Many energy storage techniques exist and can be classified by their type of storage: mechanical, electrical, electrochemical, thermal and potential. Specific techniques such as pumped hydro storage, compressed air storage, flywheel energy storage, thermal energy storage, super capacitors and superconducting magnetic energy storage have been discussed [12, 13].

Electrochemical storage using batteries has been in practice for many years, in different forms. Primary batteries are not capable of recharging and have limited application. Although, due to their simplicities and reliabilities, these are the most common form of battery. Secondary batteries, capable of recharging, have been present 
in vehicles in the form of lead acid $(\mathrm{PbA})$ batteries. $\mathrm{PbA}$ battery technology is readily available at low cost, and is ideal in vehicles for low power demand applications such as starting, lighting and ignition sequences. New secondary battery technologies such as nickel cadmium ( $\mathrm{NiCd}$ ), and nickel metal hybrides ( $\mathrm{NiMH}$ ), have been implemented in electric vehicles and hybrid vehicles [14]. These batteries exhibit higher energy and power densities than $\mathrm{PbA}$ batteries. These advantages have lead to the commercialization of all these new battery types.

Another secondary battery chemistry is lithium-ion. The process of energy storage in a li-ion battery can be briefly described using the schematic shown in figure 1.3. During discharging, lithium ions diffuse from the anode to the cathode across an electrolyte separator, while releasing an electron across an external electric circuit. During charging, the process is reversed. This will be elaborated in chapter 4. Research is ongoing to improve the electrochemistry of li-ion batteries and their performance $[15,16]$.

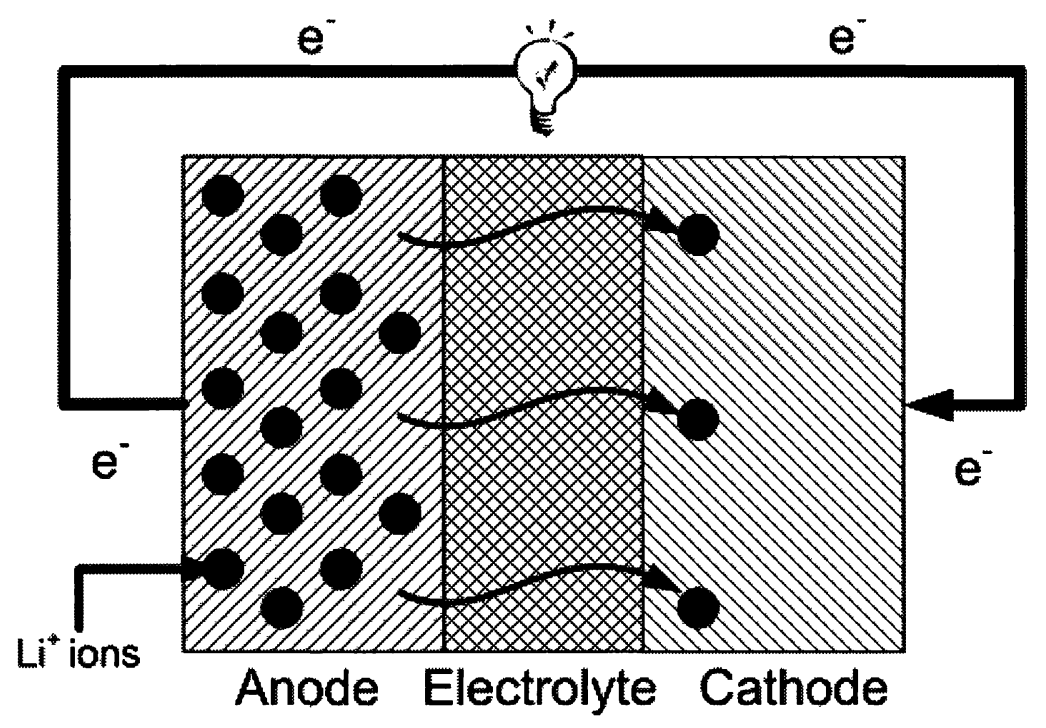

Figure 1.3: Schematic of lithium ion battery.

Li-ion batteries exhibit better characteristics compared to other secondary batteries [17]. They currently exhibit capacity retention after more than 3000 cycles at a discharge depth of $80 \%$ [18]. This value is optimistic and is contested by some [19]; it depends highly on the treatment, control and discharge depth of the battery. Li-ion batteries also have low self-discharge rates of $2 \%$ to $8 \%$ per month [20]. Even at 
low states of charge (SOC), li-ion batteries can be left for extended periods without recharging [14]. Li-ion batteries also have higher energy and power densities than other secondary batteries [17].

Ibrahim et al. [21] uses a performance index, including cost, to determine the optimal storage technique for a permanent application, similar to the criteria for RES. He concludes that while li-ion batteries exhibit better performance than other secondary batteries, they are currently too expensive. Li-ion batteries are currently available at around $\$ 1000$ USD per kWh of storage capacity [22]. Cost effectiveness will be achieved with further development, production in mass quantities and an increase in market competition. The United States Council for Automotive Research LLC has set near and longterm goals for li-ion costs at $\$ 150 \mathrm{USD} / \mathrm{kWh}$ and $\$ 100$ $\mathrm{USD} / \mathrm{kWh}$, respectively [23].

Along with cost, a relatively complex battery management system (BMS), involving a sophisticated control must be implemented to safely modulate the battery. Proper control of a li-ion battery is crucial to ensure high capacity retention, while avoiding physical dangers such as fire or explosion.

Storing electricity in a rechargeable battery facilitates a quick transient response to a varying imposed electric load. This makes micro-cogeneration systems with RES a potential solution for small-scale power generation, at a large rate of penetration.

\subsubsection{RES modelling}

Micro-cogeneration models using battery storage for load levelling applications have been developed using $\mathrm{PbA}$ batteries [24]. An electric storage model in the building simulation program ESP-r [25], using $\mathrm{PbA}$ and vanadium redox flow batteries (VRFB) has also been developed. This model, developed by Ribberink and Wang [26], incorporates a PbA and VRFB storage system, coupled with photo-voltaic (PV) renewable generation, within the power modelling domain of ESP-r, described by Clarke [27].

From the results of Annex 42 of IEA/ECBCS [1] and in conjunction with National Research Council's Institute for Chemical Process and Environmental Technology (NRC-ICPET), a li-ion battery model based on the literature and previous work was developed. This model was coupled with existing micro-cogeneration models in ESP-r [25]. The RES model developed in this work used a "grey box approach", practiced extensively in engineering fields $[1,27]$. This approach treats each sub-system as 
control volumes for energy transfer, and where conservation of mass and energy laws apply. A schematic of the RES model using this control volume method is shown as figure 1.4.

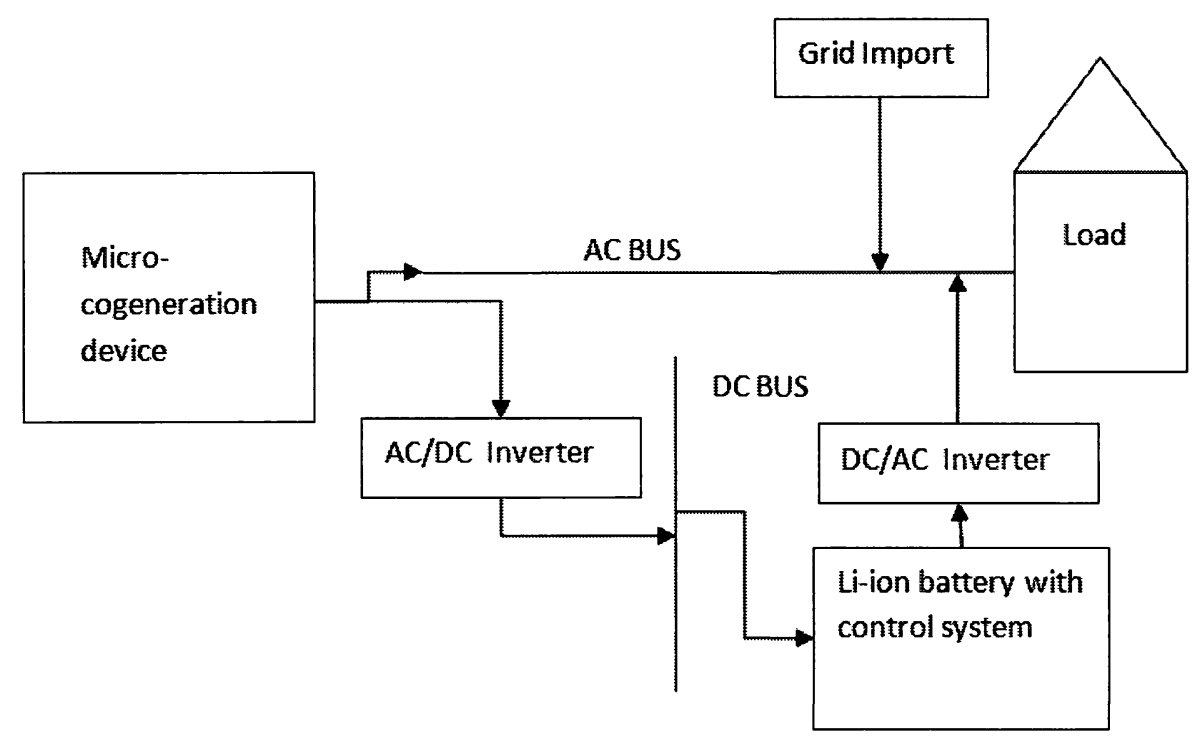

Figure 1.4: Schematic of Li-ion micro-cogeneration model.

For any simulation result to be valid, not only should the physics of the real life system be captured, but the simulation inputs also must be accurate. In the context of RES, one of these inputs are the electrical demands of the household.

\subsubsection{Residential load monitoring}

Whole building simulation software has been developed to accurately assess thermal and electrical demands of a building. This assumes that accurate inputs for occupant activities, such as appliance usage and lighting, have been provided. These activities contribute to the household's non-HVAC loads. These loads, typically applied in simulation software as energy profiles, can have large effects on the building's thermal and electrical demands. Non-HVAC loads vary widely between households, depending not only on the size and location, but on the habits, lifestyle and social status of the occupants [28]. 
Accurate data to represent typical non-HVAC loads for a wide spectrum of Canadian dwellings is unavailable [1]. This makes it difficult to assess electric demands in building simulation software. The results from Annex 42 of the IEA/ECBCS showed that the potential for residential cogeneration is reliant on these non-HVAC loads [1]. Previous work in producing synthetically generated non-HVAC profiles for Canadian houses using a bottom-up approach was performed by Armstrong et. al [29]. These profiles did not include air-conditioning usage and will be discussed in further detail in chapter 3.

Beausoleil-Morrison [30] observed that occupant intervention can have significant impacts on space cooling. Space heating in the winter is easy to predict in building simulation, as occupant intervention has little effect. However during the summer, occupant intervention from opening and closing windows, blinds and shutters have significant impacts on cooling loads by bringing in outdoor air and reducing passive solar gains. Thermostat control strategies, chosen by the occupants, also affect space cooling. These occupant interventions make space cooling predictions in building simulation difficult. Further data regarding electrical usage and occupant intervention of typical Canadian dwellings is needed to assess a RES system.

\subsection{Research objectives}

Canadian residential electricity usage from appliances and air-conditioners, i.e., peak demand, leads to inefficient electricity production from increased GHG emissions. New strategies in micro-cogeneration and electrical storage may be a solution to reducing peak demand. These solutions, that can be assessed via building simulation, require accurate representation of non-HVAC and air-conditioning loads for valid results. This thesis will attempt to address the above with the following research objectives:

- Improvement of non-HVAC electrical load profiles for Canadian households;

- Improvement of space cooling electrical profiles and their dependency on occupant habits for Canadian households;

- Development of a quasi-steady-state li-ion battery model in ESP-r;

- Calibration of the li-ion model parameters with external data; 
- Demonstration of new modelling capabilities of the li-ion model coupled with a cogeneration model within a residential house with non-HVAC and space cooling loads; and

- Recommendations toward a full assessment and optimization of such a system, and the metrics to measure its performance.

\subsection{Thesis outline}

To meet the objectives listed in the previous section, the remaining thesis will be organized in the following fashion:

- Chapter 2 reviews the ESP-r methods used to simulate the thermal and electric demands of a building.

- Chapter 3 reviews the non-HVAC electrical consumption data collected in a measurement project of residential dwellings in the Ottawa area. This data will be compared to previous methods of representing non-HVAC loads in a Canadian context. The chapter also reviews the space cooling electrical consumption data collected in the same project.

- Chapter 4 briefly reviews li-ion battery chemistry and the different approaches in modelling this behaviour. The chapter proceeds with the development and implementation of a li-ion battery model within ESP-r.

- Chapter 5 outlines the procedure to calibrate a li-ion battery model in ESP-r based on specific battery chemistry, configuration, etc.

- Chapter 6 reviews the summation of the previous chapters by demonstrating the li-ion model coupled with a micro-cogeneration model within a residential house with non-HVAC and space cooling loads.

- Chapter 7 summarizes the results of this research and recommends future work needed to assess a RES system. 


\section{Chapter 2}

\section{ESP-r simulation methods}

The li-ion modelling research objectives could be completed using many simulation tools. The use of numerical method software such as $\mathrm{C}++$ or Matlab would first require the modelling of the thermal, air flow, plant and electrical interactions that occur within a dwelling. The use of building simulation software, where these interactions have been previously modelled, would eliminate this problem. While other building simulation software such as TRNSYS or EnergyPlus could have been used, ESP-r [2.5] has been chosen for this work because it is a non-proprietary, open source development software and has been extensively validated [31].

The development of ESP-r and its ability to simulate the thermal demands of a building began in the 1970's. Since then it has been continuously expanded and refined by developers around the world. More facets of building simulation such as air flow, electrical networks and plant networks have been implemented. [27].

Understanding the methods used to simulate in ESP-r is necessary for interpreting its outputs, and for designing and implementing a li-ion model. The following description of ESP-r's methodologies are adapted from previous works that are cited in the individual sections. Section 2.1 briefly outlines the thermal, air flow and plant networks, while the power flow network is discussed in section 2.2 .

\subsection{The ESP-r thermal, air flow and plant net- works}

The following description of ESP-r's thermal, air flow and plant networks is adapted from Clarke [27]. ESP-r uses a finite difference approach to discretize a building into 
sections, known as zones. A zone can be a single room of a building, or several rooms if their thermal states are assumed to be similar enough that they can be lumped together for computational ease.

The zones are discretized into nodes. By default, each zone is discretized into one air point node. The zone is bounded by opaque and non-opaque constructions, i.e., walls and windows, respectively, which are also discretized into nodes. Any homogeneous construction is discretized into three nodes: one at either surface and one interior node. A wall made of $n$ layers, e.g., gypsum, air gaps, brick etc., will be discretized into $2 n+1$ construction nodes.

Each node is treated as a control volume and has the laws of conservation of energy and momentum applied over it to determine its thermal state at any time-step. In general, the thermal storage capacity of the node with respect to time must equal the net energy flow paths to the node and the net energy generation at the node:

$$
m C_{p} \frac{d T}{d t}=\dot{q}_{p a t h n e t}+\dot{q}_{g e n ~ n e t}
$$

where $m$ is the mass of the wall construction or the air in the zone in $k g, C_{p}$ is the heat capacity of the wall construction or the air in the zone, in $J \cdot \mathrm{kg}^{-1} \cdot \mathrm{K}^{-1}, T$ is the temperature of the wall construction or the air in the zone in Kelvin, and $\dot{q}$ is energy transferred to the node with respect to time in Watts. Figures 2.1 and 2.2 illustrate the energy flow paths and the energy generation considered at an air point node and at a construction node, respectively.

For an air point node, the net energy flow paths are made up of several contributions. The contribution from advective mass transfer, i.e., conservation of momentum, is the infiltration from other zones, and the infiltration with the exterior through cracks and openings. This contribution is proportional to the mass flow rate and the temperature gradient between the zones and the exterior. For example, the advective mass transfer contribution to air point node $i$ of a zone, in a building model with $n$ zones and no connection to the exterior would be:

$$
\dot{q}_{\text {net advectoon }}=\sum_{\jmath=1}^{n-1}\left(\dot{m}_{\jmath \rightarrow 2} C_{p}\left(T_{\jmath}-T_{\imath}\right)\right)_{\jmath \neq \imath}
$$

where $\dot{m}_{\jmath \rightarrow \imath}$ is the mass flow rate between zone $j$ and $i$, in $k g \cdot \mathrm{s}^{-1}$. The mass flow 


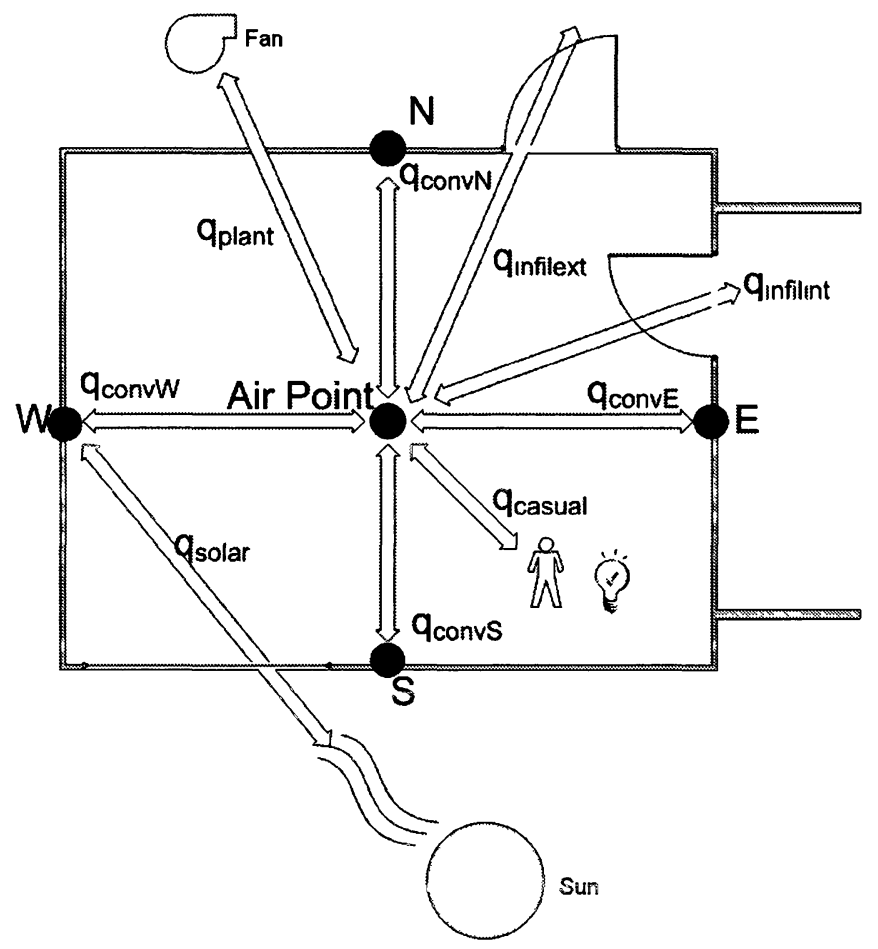

Figure 2.1: Energy flow paths and energy generation at an air point node in ESP-r.

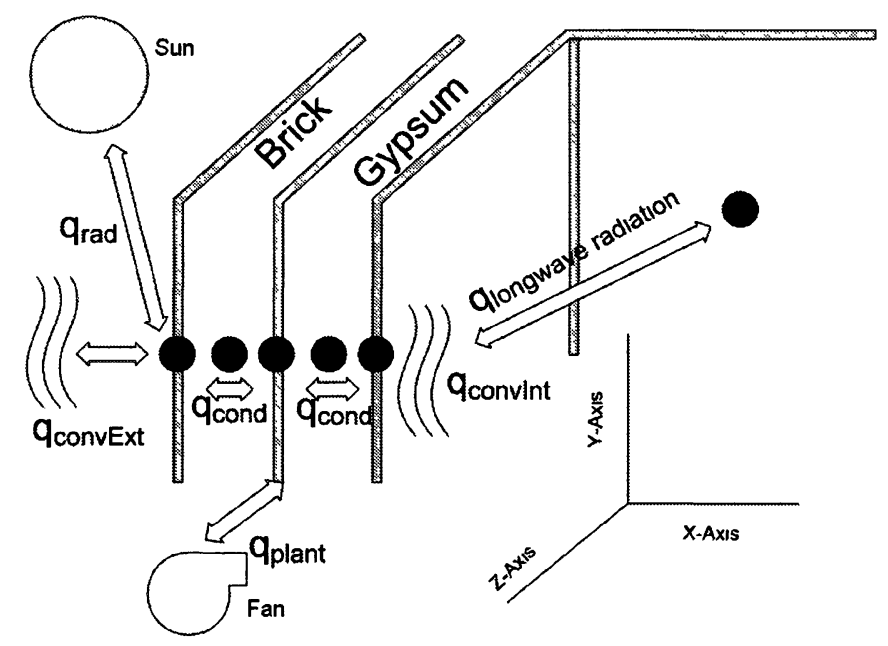

Figure 2.2: Energy flow paths and energy generation at construction nodes in ESP-r. 
rate is governed by the pressure gradient between the zones or exterior, scaled by an advection coefficient. From the conservation of mass law, the sum of the mass flows in a building with $n$ zones must be zero (see eq. 2.3).

$$
\sum_{\jmath=1}^{n} m_{\imath \jmath}=\sum_{\jmath=1}^{n} f\left(\Delta P_{\imath \jmath}\right)=0
$$

where $\Delta P_{\imath \jmath}$ is the pressure gradient between zone $j$ and $i$, in $k P a$. The infiltration from outside the building is specified using a schedule, either in rate of volume with respect to time, or in air changes per hour $(\mathrm{ACH})$. Recent work has introduced the Alberta Infiltration Model (AIM) which calculates the infiltration based on pressure gradients and wind speeds from an imposed weather file [32].

The contribution from heat transfer, i.e., conservation of energy, is the convection from the bounded surfaces of the zone. This contribution is proportional to the temperature gradient between the surface node and the air point node. For example, the convective heat transfer contribution to air point node $i$ of a zone in a building model, bounded by $m$ surfaces would be:

$$
\dot{q}_{\text {net convection }}=\sum_{s=1}^{m} A_{s} h_{s \rightarrow \imath}\left(T_{s}-T_{\imath}\right)
$$

where $A_{s}$ is the surface area of surface $s$, in $m^{2}$, and $h_{s \rightarrow \imath}$ is a convection coefficient between surface $s$ and air point node $i$, in $W \cdot m^{-2} \cdot K^{-1}$. There is no radiation exchange and insignificant conduction exchange at an air point, hence, these contributions are negligible. The net energy generation terms in equation 2.1 include any contributions from the HVAC plant system and casual gains from occupants and appliances in Watts. The advection and convection correlations used in the above equations are determined from empirical observations.

Construction nodes follow the same form of equation 2.1 above. The net energy flow paths of internal construction nodes, i.e., nodes that are not on the inner or outer surface of a wall, are only made up of conduction from adjacent nodes. In the case of air gaps in the wall, convection will exist as well. The net energy flow paths of external construction nodes are made up of conduction from adjacent inner nodes, convection from air point nodes or from the exterior, and long wave radiation exchange with other surfaces or radiation exchange with the exterior. The conduction through a construction, in Watts, is proportional to the temperature gradient over the 
construction and its material conductivity, in $W \cdot m^{-1} \cdot K^{-1}$. A linearized radiation coefficient $h_{r}$, in $W \cdot m^{-2} \cdot K^{-1}$, is used to treat the radiation exchange between a surface $s$ and the other surfaces in the zone:

$$
\dot{q}_{\text {net radıatıon }}=\left(\sum_{\imath=1}^{m-1} A_{s} h_{r s \rightarrow \imath}\left(T_{s}-T_{\imath}\right)\right)_{\imath \neq s}
$$

The net energy generation term in equation 2.1 for the construction nodes include any contributions from an HVAC plant system injected into the construction in Watts.

Non-opaque surfaces, i.e., windows, are discretized in the same fashion as opaque ones. However, in the presence of incoming solar radiation, ESP-r calculates the amount of radiation transmitted into the zone and absorbed by the surface itself. This is specified over several angles relative to the window. Recent work has advanced the non-opaque surface treatment to include the effects of convection and diffusion of thermal heat to the surface from absorbed radiation [33].

The plant network does not need to be defined at all. In this case, ESP-r simply calculates the required thermal loads of the building over the time-step. If the plant network is defined, another set of nodes is created to explicitly model an HVAC system. Each node represents a component of the HVAC system, e.g., fans, compressors, coils, etc., similar to figure 23 .

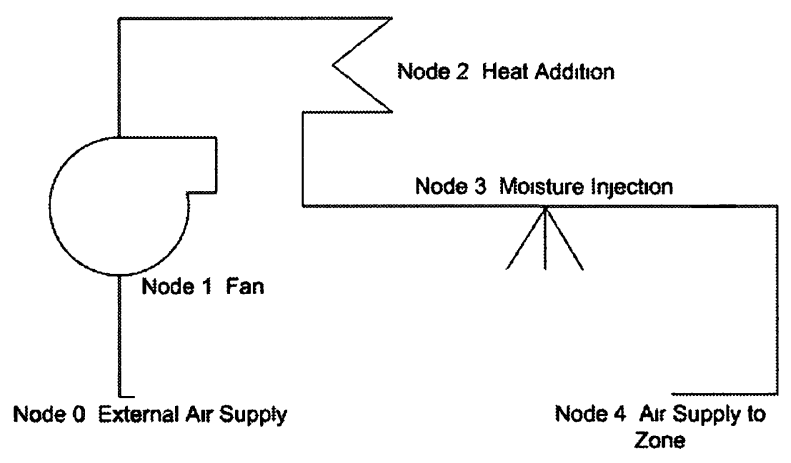

Figure 2.3: Schematic of example HVAC system in ESP-r.

The laws of conservation of energy, mass and momentum are applied over the nodes to determine the energy state of the parameters entering and leaving the nodes. The plant network can be defined in increasing levels of complexity, increasing accuracy and computation time as well. 
The above equations are expanded using a first order Taylor series expansion. This may be done using an explicit or implicit scheme, i.e., if the equations use the previous and current time-step values (explicit), or the future and current time-step values (implicit). Each scheme has draw backs; explicit schemes are stable but heavily dependant on the time-step resolution, and implicit schemes tend to be unstable. ESP-r employs a Crank-Nicolson approach which is a 50:50 weighted average of both schemes to solve the system of equations.

Each nodal equation is arranged to create three arrays in the form $A \cdot T=B$. The matrix is closed with the boundary conditions imposed on the system, i.e., the temperature control set-points of the zone and/or control set-points on the plant. The $A$ matrix contains the conduction/convection/radiation coefficients between nodes, and the $B$ matrix contains the boundary condition terms. The $T$ matrix is a linear vector of nodal temperatures and, if applicable, a net plant heat injection/removal term. Figure 2.4 shows a thermal network set of arrays and focuses on node $i$, an interior construction node with the plant contributing to its thermal state. Using Gaussian elimination, the $T$ vector is solved to determine the nodal temperatures and plant contribution for the time-step.

To obtain a global solution of the nodal temperatures and plant contribution, the different networks must be solved simultaneously. This is because several of the parameters are needed in more than one set of equations. For example, a microcogeneration device would affect the thermal, plant and power flow networks. Consequently, the networks are highly coupled and various parameters are passed between. The solution process occurs using an iterative method until all the network solutions have converged. The interested reader is referred to Clarke [27] for more information on the development of the networks in this section.

\subsection{Electrical power flow network in ESP-r}

The previous section briefly described the basic domains that ESP-r implements to calculate the energy performance of a building. The electrical power flow network of ESP-r need not be defined to calculate the thermal loads of the building. However, the objectives of this research require the implementation of this network, so it is necessary to outline its development. The following is a brief overview of the power flow network derived by Kelly [34]. The interested reader is referred to Kelly's thesis 


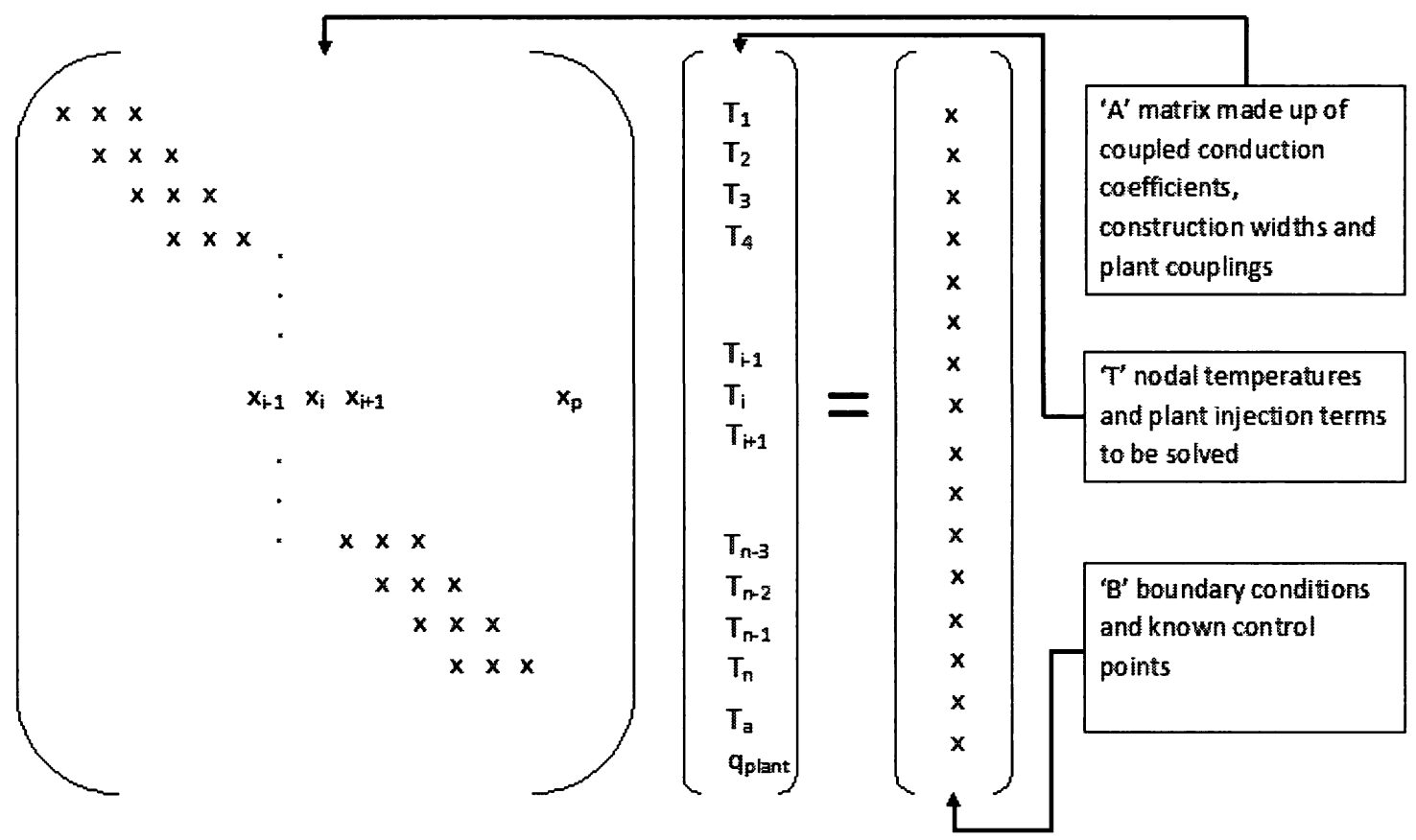

Figure 2.4: Matrix equations for thermal domain at $i^{\text {th }}$ node with plant injection.

[34] or a paper by Clarke [35] for further description of the power flow network.

The power flow network in ESP-r uses a series of nodes to represent electrical components in a building. The network must be capable of expressing alternating current (AC) and direct current (DC). As such, the following derivation will be in terms of phasor values. In other words, the current and voltage are changing direction sinusoidally with respect to time. The impedances in a network can be real (resistors), imaginary (capacitors and inductors), or a combination of both.

Each node represents the electrical current contribution of a component over a time-step. Kirchoff's law that states that the sum of the currents at any node in an electric network must be zero. Hence at node $i$ in a network of $n$ nodes, it follows that:

$$
\left(\sum_{j}^{n} \widetilde{I}_{i j}\right)_{j \neq i}=0
$$

where $\widetilde{I}_{i j}$ is the phasor current between nodes $i$ and $j$ over the time-step in Ampere. 
An example of an electric power flow network defined in ESP-r is shown in figure 2.5 .

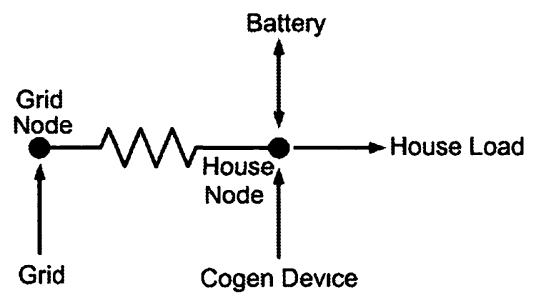

Figure 2.5: Example of electrical network schematic in ESP-r.

In the network, components can take on one of three roles: electricity consumers, electricity generators, and electricity transmitters. From the three types of components above, Kirchoff's current law in equation 2.6 still holds :

$$
\left(\sum_{\jmath=1}^{C n} \widetilde{I}_{C \imath \jmath}\right)_{\jmath \neq \imath}+\left(\sum_{\jmath=1}^{G n} \widetilde{I}_{G \imath \jmath}\right)_{\jmath \neq \imath}+\left(\sum_{\jmath=1}^{T n} \widetilde{I}_{T \imath \jmath}\right)_{\jmath \neq \imath}=0
$$

For example, figure 2.5 has two nodes defined: a node representing the grid contribution and a node representing electrical interactions to the house. The grid node has one source component, the grid itself, and one transmission component, the electricity transmitted to and from the house. From equation 2.7 above, the grid node would reduce to:

$$
\widetilde{I}_{G g r i d}+\widetilde{I}_{T \rightarrow h o u s e}=0
$$

Likewise for the house node, the generating component is the micro-cogeneration device, the consuming component is the house's electrical loads, and the transmitted component is the interaction between the grid. The battery at the house node can act as a generator or consumer depending on if the battery is charging or discharging:

$$
\widetilde{I}_{\text {Gcogen }}+\widetilde{I}_{\text {Chouse }}+\widetilde{I}_{C / \text { Gbattery }}+\widetilde{I}_{T \rightarrow \text { grld }}=0
$$

All the generating and consuming terms are moved to the right hand side of the equation and Ohm's law is substituted in the transmission terms. Ohm's law states that the ratio between the voltage and current, in magnitude and phase, is known as 
the impedance, measured in $\Omega$. This transforms the house node, equation 2.9, to:

$$
\frac{\widetilde{V}_{\text {house }}-\widetilde{V}_{\text {grid }}}{\widetilde{Z}_{\text {house } \rightarrow \text { grdd }}}=-\widetilde{I}_{\text {Gcogen }}-\widetilde{I}_{\text {Chouse }}+-\widetilde{I}_{C / \text { Gbattery }}
$$

where $\widetilde{Z}_{\text {house } \rightarrow \text { grd }}$ is the complex impedance between the grid node voltage and the house node voltage.

Through some further manipulation and the transformation to complex power, which is the product of complex voltage and the conjugate of complex current, an equation set is extracted. This set of equations is arranged into a solvable set of arrays. The right hand side of this array contains all the generated and consumed devices at each node, and the left hand side contains the impedance values between the transmitting nodes. This set is closed by specifying a reference voltage for the network, typically the voltage of the grid. The array can then be solved for each nodal voltage and phase angle at any time-step. Once again, the interested reader is referred to Kelly's thesis [34] or the paper by Clarke [35] for further description on the development of the power flow network.

It is necessary to determine, at any time-step, the power contribution from the battery to send to the electric network. Referring to figure 2.5, along with the contribution of the battery, another requirement is the representation of non-HVAC electrical loads and space cooling loads in ESP-r. Accurate representation of these loads is necessary for a valid assessment of RES. The following chapter reviews a measurement project aimed at this objective. 


\section{Chapter 3}

\section{Non-HVAC and Space Cooling Electric Loads}

Chapter 2 briefly reviewed the methods applied in ESP-r to solve the energy interactions that take place in a building. The electrical network, explained in section 2.2, will be used to assess a RES system. The validity of this assessment requires accurate representation of residential electric loads in building simulation. This chapter reviews an ongoing project in representing these loads in a Canadian context. Sections 3.2, 3.3 and 3.4 represent the contributions of this research in the gathering and analysis of Canadian residential electricity usage.

\subsection{Previous work and synthetically generated profiles}

Representing non-HVAC electrical residential loads for different countries and climates has been previously performed. The approach used to represent them generally falls into two categories: empirical data collection at the end user level or a bottom-up approach.

The following are some examples of empirical data collection projects occurring worldwide. A study by Isaacs et al. at the BRANZ Group monitored 400 randomly selected houses in New Zealand from 1997 to 2005 [36]. Each house, monitored for at least 11 months, had the rate of each type of fuel (e.g., natural gas, electricity etc.) entering the house recorded at 10 minute intervals. The temperature of each room in the household was also measured. Information of the occupants' social status and physical properties of the house were also recorded. 
Another study by Parker at the Florida Solar Energy Center monitored 204 residences in Central Florida in 1999 [37]. This study monitored total electricity consumption at 15 minute intervals, broken down into space heating, space cooling, water heating, dryers, cooking and pool energy use.

Subtask A of Annex 42 of IEA/ECBCS [28] reviewed residential non-HVAC electricity profiles gathered around European and North American nations. Most notedly, was the monitoring of non-HVAC profiles of 90 homes around the United Kingdom at 5 minute intervals between 2002 and 2005. Other collaborating nations included Switzerland, Finland, Belgium, Germany and Portugal.

Within Canada, a study by Hydro-Québec from 1994 to 1996, measured nonHVAC electricity demand from 57 single detached homes at 15 minute intervals [28]. Another study by Newsham at the National Research Council's Institute for Research in Construction (NRC-IRC) and Rowlands at the University of Waterloo [38] recorded hourly electrical utility consumption at the household meter. It included 1297 houses in Southeastern Ontario from 2006 to 2008, of which detailed information of the household and occupants for 365 houses is known, 498 houses from Eastern Ontario from 2006 to 2007, and 150 houses from Northwestern USA from 2005 to 2008.

Empirical data collection projects are the most effective way of representing nonHVAC electric loads. However, they are often expensive and intrusive to the occupant, making it difficult to achieve large sample populations. None of the projects above measured non-HVAC or space cooling electricity consumption of Canadian residences at a time resolution finer than 15 minutes.

Subtask A of Annex 42 of IEA/ECBCS [28] noted that a coarse time resolution may fail in effectively capturing electric loads. Figure 3.1 illustrates the necessity of having electricity usage data at a fine time resolution. The data in the figure was collected in this work, explained in section 3.2. The figure shows the average power drawn by the household of each minute in the day, and the same data using hourly averaged values in the day. If the hourly curve was used for a simulation of a cogeneration device, the device would only have to satisfy a maximum load of $1.5 \mathrm{~kW}$. If the one minute curve was used for the same simulation, the device would have to meet several periods approaching $4 \mathrm{~kW}$ of electricity demand, much closer to the household's instantaneous electricity demand. For the assessment of a micro-cogeneration device with RES, a temporal resolution of five minutes or less would allow the physics of such a system to take place with sufficient resolution, 


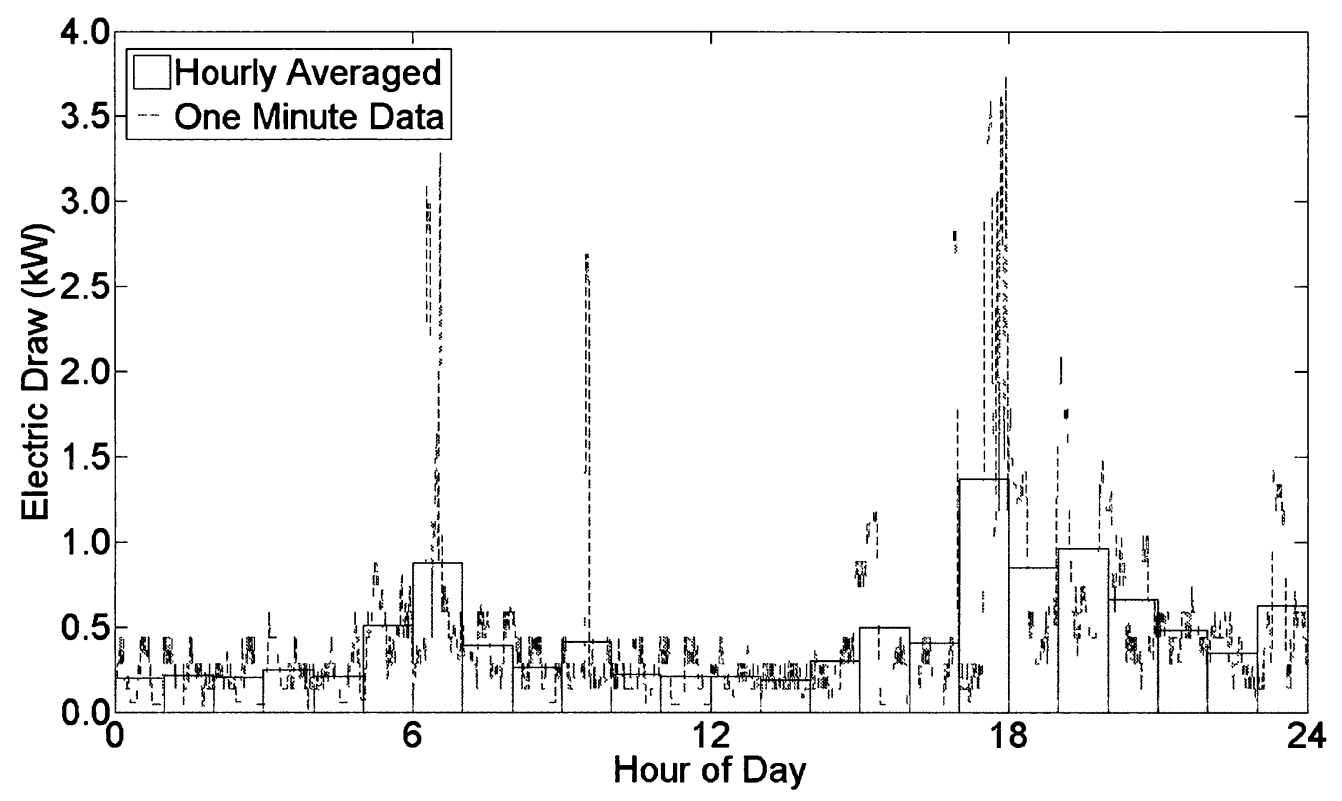

Figure 3.1: Comparison of typical electricity profile taken at one minute intervals and averaged over one hour intervals.

whilst conserving quasi-steady-state assumptions used in modelling transient plant performance as described by Beausoleil-Morrison [39].

The bottom-up approach is the second method for representing residential electricity usage. This approach uses statistical data of annual household electricity use and household appliance penetration rates as inputs to an algorithm. Using stochastic random number generation, electricity usage profiles are created based on the probabilities of appliance usage applied in the algorithm.

The bottom-up approach obtains data relatively easily from energy production by source data and census information. However, the approach relies heavily on the accuracy of the algorithm implemented for generation. Examples of the bottom-up approach include the works by Widen et al. in Sweden [40], Capasso et al. in Italy [41] and Paataro and Lund in Finland [42].

As discussed in section 1.2.5, synthetically generated electricity profiles, representing the stock of Canadian households, using a bottom-up approach were developed by Armstrong et. al [29]. The non-HVAC profiles, created at five minute intervals, targeted three levels of annual electricity consumption: low, medium and high. These targets were based on statistics of Canadian residential electricity use [2, 3]. Each 
consumption category had three years of non-HVAC profiles produced. This work did not include air-conditioning usage in its development. The profiles produced in this work were validated with the dataset collected by Hydro-Québec from 1994 to 1996 [28].

Both approaches, empirical and bottom-up, generally result in attempts to create new profiles based on original data and the use of stochastic randomly generated numbers. This requires the implementation of some algorithm to mimic occupant behaviour and appliance usage. Earlier methods in this began with works by Walker and Pokoski [43], and Gross and Galiana [44], and have recently developed into more sophisticated algorithms using fuzzy logic, genetic algorithms and neural networks [45]. Paatero and Lund [42] noted that these new algorithms may improve profile accuracy where detailed statistical data is unavailable.

\subsection{Electrical measurement project}

To better understand Canadian residential non-HVAC and space cooling electricity use at a fine time resolution, electric consumption data was measured in several houses throughout Ottawa, Ontario for one year beginning in July, 2009. Measuring devices were installed at the electric panels of twelve houses similar to the images shown in figure 3.2. These devices individually measured the household's overall electricity consumption, air-conditioning consumption and furnace air circulation fan consumption at one minute intervals. The households in the project obtained space heating and DHW by other means than electricity. Thus, by subtracting the air-conditioning and furnace fan consumption from the overall house electrical consumption, the nonHVAC electrical consumption was known.

The households were selected in an effort to represent the wide spectrum of Canadian dwellings. They were chosen to fit in one of the following annual energy consumption categories: low, medium and high. These categories were taken from the annual consumption targets used by Armstrong et. al [29] in her development of synthetically generated profiles.

Periods of data are missing from the households throughout the year, elaborated upon further. To determine the approximate annual non-HVAC electricity consumption of a household, in $k W h \cdot y r^{-1}$, the average household electric draw from the 

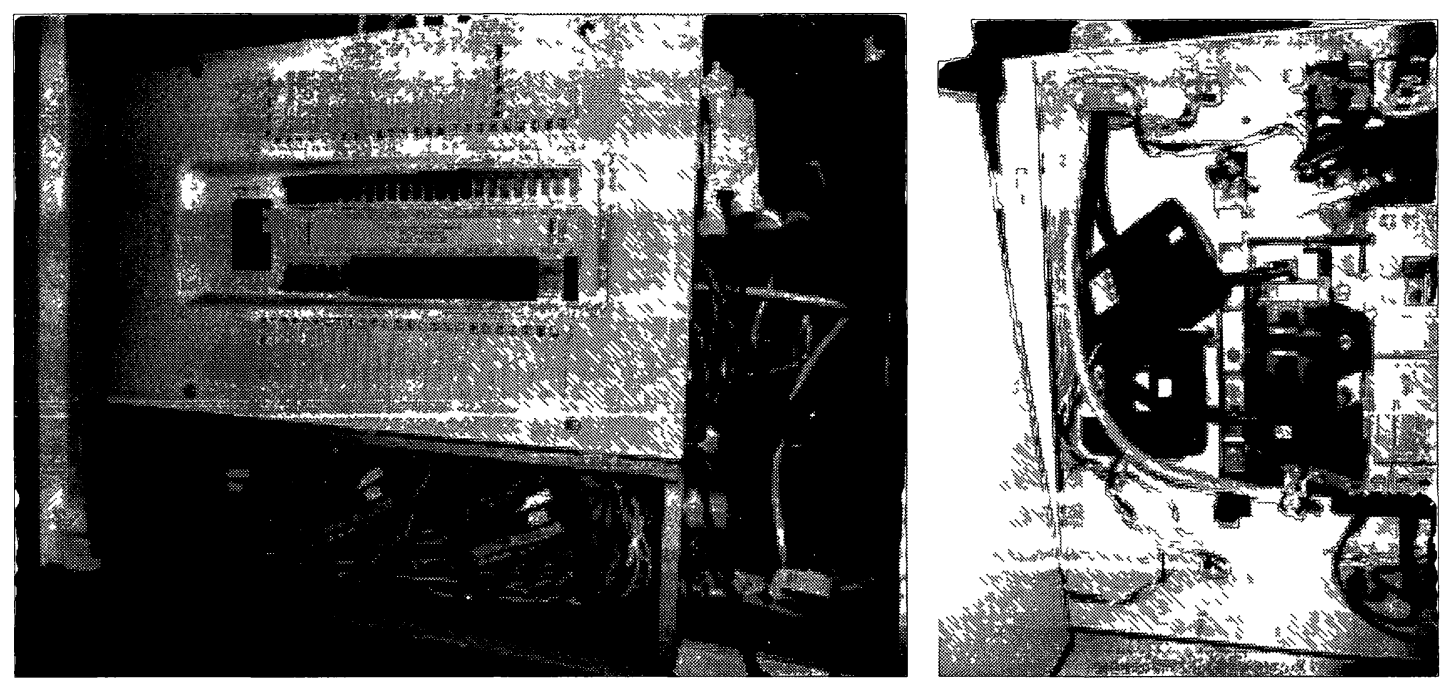

Tigure 3.2: Neasuring devices installed at une electric panel of a participant in the electric measuremerat project.

availlable data was determined, in kWW, and was assumed to have been drawn constantly by the household for one year. Additionally, at the time of analysis, a full year of data was not yet collected. More than one month of data, beginning near the end of May 2010, was missing for each household. The approximate annual nonHVAC electricity consumption of each house, sorted by energy consumption category, is summarized in table 3.1 .

\begin{tabular}{cc|cc|cc}
\hline Label & Low & Label & Medium & Label & High \\
\hline \hline $\mathbb{H 1}$ & 4870 & $\mathbb{H} 5$ & 5557 & $\mathbb{H 9}$ & 10120 \\
\hline $\mathbb{H} 2$ & 2641 & $\mathbb{H} 6$ & 6373 & $\mathbb{H 1 0}$ & 8877 \\
\hline $\mathbb{H} 3$ & 4669 & $\mathbb{H} 7$ & 5155 & $\mathbb{H 1}$ & 8847 \\
\hline $\mathbb{H} 4$ & 5044 & $\mathbb{H} 8$ & 9328 & $\mathbb{H} 12$ & 11257 \\
\hline
\end{tabular}

Table 3.1: Annual electric consumption of the participating households in $k W h$. $y r^{-1}$. Note that bias exists due to the lack of a complete year of collected data. 
Three households, H1, H10 and H12, each had an additional three electric appliances measured. These were the stove, dryer and dishwasher. This was in collaboration with NRC-IRC to gain a better understanding of high energy consumption appliances in households. H10 used electricity for DHW and had this circuit monitored instead of their dishwasher. All of the occupants in the project also completed a brief survey prior to the project's commencement. This survey included questions about the number of occupants, their typical physical presence over the day, their thermostat control, and their willingness to use shading and window openings to mitigate cooling loads throughout the day.

In an oversight, one of the medium houses, labelled $\mathrm{H} 8$ in table 3.1, obtained DHW from an electric heater that was not being measured. While this is still an interesting case to study in building simulation and reducing peak electrical loads, for the non-HVAC analysis in this chapter, this house is removed. The annual electricity consumption reported for $\mathrm{H} 8$ in table 3.1 includes electricity consumed for DHW heating.

\subsubsection{Measurement methods and data analysis}

\section{Measurement methodology}

Energy transducers (ETs) monitored multiple circuit phases, at a rate of four hertz, and logged the electricity consumed by the circuit each minute. Current transformers (CTs) were attached to a desired circuit at the electric panel. If an AC current existed in the circuit that the CT was wrapped around, a current from the circuit's magnetic field would be induced in the CT. The voltage of the desired circuit, and phase angle between the voltage and current, were also sampled. Using the current induced in the CT, and the voltage and phase angle of the circuit, the energy consumed by the electric circuit, in $W \cdot h$, was determined by the ET using equation 3.1.

$$
E=V I \cos \theta \Delta t
$$

where $V$ and $I$ in this equation are the root mean square of their phasor counterparts, $\theta$ is the phase angle, and $\Delta t$ is the logging interval ( 1 minute).

Each CT, classified by their rating, had a specific threshold of energy consumption, shown in table 3.2 . 


\begin{tabular}{cc}
\hline Rated CT Size(Amps) & Watt-hours per pulse \\
\hline \hline 30 & 0.750 \\
\hline 50 & 1.250 \\
\hline 100 & 2.5 \\
\hline
\end{tabular}

Table 3.2: Watt-hours per pulse resolution in logging equipment in the measurement project.

The rated current of the CTs on the overall household circuit was $50 \mathrm{~A}$, and 30 $A$ for the individual circuits. Household $\mathrm{H} 4$ in table 3.1 had $100 A$ CTs on its overall circuit and $50 A$ CTs on its individual circuits.

If the circuit being monitored consumed this threshold of energy, a relay switch would open in the ET. Using the sample rate of four hertz, if the relay was open over the last sampling cycle, the logger would increase its count of open relay occurrences, also known as "pulses", by one. At the end of each logging interval, the total number of pulses was registered by a data logger. Thus for a logging interval of one minute and a sample rate of four hertz, a maximum of 240 pulses per logging interval could be registered by the data logger. The pulses could be converted to average electricity drawn over the last logging interval of one minute using equation 3.2.

$$
\text { Power }(k W)=0.06 \cdot \text { WattHoursPerPulse } \cdot \text { PulseCounts }
$$

\section{Measurement uncertainty}

The error on the data needs to be addressed prior to any interpretation. Using a root sum method accepted by the American Society of Mechanical Engineers [46, 47], the bias error on the energy measured can be determined. The equipment manufacturer reported bias errors on the CTs, phase angle $\theta$, and the ET. These are listed in table 3.3. The bias error reported on the ET by the manufacturer was independent of the bias error on the CT. Hence, the root sum method was assumed to be able to assess the error in the energy consumed by the circuit.

It should be noted that these values were for normal operation. The error increased if the current in the circuit was out of the rated range of the $\mathrm{CT}$, or if the phase angle was too large. The acceptable current range was $10 \%$ to $130 \%$ of the rated current 


\begin{tabular}{cc}
\hline Device & Bias Error $\beta$ \\
\hline \hline CT & $\pm 1 \%$ of full scale \\
\hline$\theta$ & $\pm 2^{\circ}$ \\
\hline ET & $\pm 0.5 \%$ of reading \\
\hline
\end{tabular}

Table 3.3: Bias errors of measuring devices.

of the $\mathrm{CT}$, and the acceptable phase angle was less than $60^{\circ}$.

According to the manufacturer, the error on the voltage, $\beta_{V}$, was accounted for in the error on the ET, $\beta_{E T}$. The term $\beta_{E T}$ also represents error beyond bias in the measured quantities (current, voltage and phase angle), i.e., it accounts for error on the relay in the ET. Referring to equation 3.1, the root sum square method to determine the overall error in the measurements is then:

$$
\begin{gathered}
\beta_{\text {TOTAL }}=\left[\left(\beta_{E T}\right)^{2}+\left(\frac{\partial E}{\partial I} \beta_{I}\right)^{2}+\left(\frac{\partial E}{\partial \theta} \beta_{\theta}\right)^{2}\right]^{1 / 2} \\
\beta_{\text {TOTAL }}=\left[\left(\beta_{E T}\right)^{2}+\left(V \cos \theta \Delta t \beta_{I}\right)^{2}+\left(-I V \sin \left(\beta_{\theta}\right) \Delta t\right)^{2}\right]^{1 / 2}
\end{gathered}
$$

Thus, by taking the reading of the energy consumed over the last minute, the full rated scale of the current, which is $50 \mathrm{~A}$ for the overall circuit and $30 \mathrm{~A}$ for individual circuits, and the phase angle, the error on any measurement is known. Unfortunately, the phase angle was not recorded and is difficult to assess. For typical house loads which are purely resistive, this would be close to zero degrees, making the last term's contribution in equation 3.4 negligible. For complex inductive loads such as a dryer or compressor in the air-conditioning circuit, this value would play a larger role. More analysis into the accuracy of the equipment outside acceptable current ranges and at high phase angles is necessary.

To illustrate, a plot of bias error as a function of power consumed is shown in figure 3.3. This plot is for an overall household circuit, with a voltage of $240 \mathrm{~V}$ and a $\mathrm{CT}$ with a $50 \mathrm{~A}$ rating attached to the circuit. The phase angle is assumed to be zero and the $\mathrm{CT}$ is assumed to be measuring within its rated range. Equation 3.4 is used to calculate the bias error on the consumed energy, in $W \cdot h$, which is converted to bias error on an assumed constant power drawn over the one minute logging interval, 
in $k W$. Bias errors on power drawn below $1.2 \mathrm{~kW}$ or above $15.6 \mathrm{~kW}$ would be higher than the values shown in the figure, because this would be outside the acceptable range of the $50 \mathrm{ACT}$ attached to the circuit. Again, more work in equating this error is necessary.

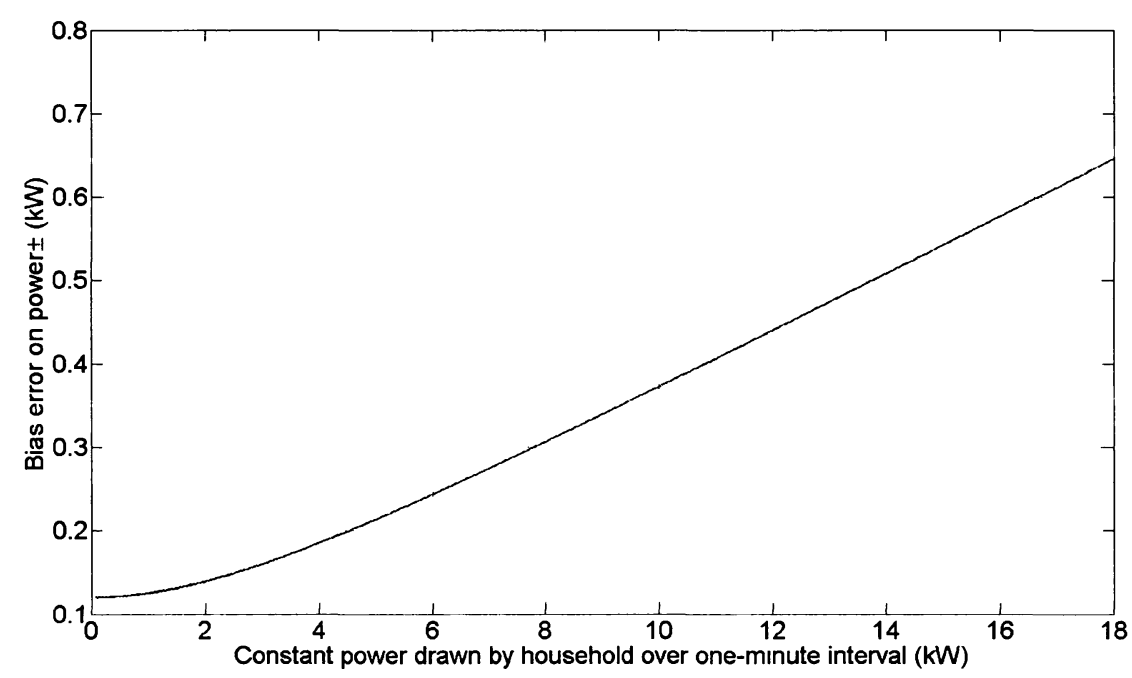

Figure 3.3: Bias error as a function of average power drawn by the entire household over one minute. Note that this is for a $240 \mathrm{~V}$ circuit with a $50 \mathrm{ACT}$ attached, and is assumed to be working within an acceptable current range and with a phase angle of zero.

The logger also reported a bias error with respect to reported logged time. Under normal operation of an environment temperature of $25^{\circ} \mathrm{C}$, this was \pm 5 seconds per week. For the houses that needed downloading once a month (discussed further on), this equated to a maximum bias error of \pm 22.5 seconds. For the houses that were downloaded once every two months, the bias error was \pm 45 seconds. This logging time error grew with decreasing temperature, up to \pm 21 seconds per week at $-20^{\circ} \mathrm{C}$. Because the majority of the houses' electrical panels were located inside the home, this larger bias error was not a factor.

Household H3 shown in table 3.1 had its electrical panel located in its garage. Consequently, its bias error on the reported logged time may be larger. The reported time stamp was truncated to the nearest minute, creating a resolution error of \pm 30 seconds. Hence, the resolution error should account for the majority of the bias error with respect to time. 


\section{Data normalization}

To compare household data sets to each other, a method of expressing the data in terms of a datum or percentage was necessary. It was thought that expressing a household's data as a percentage of its maximum power draw from the entire study period would accomplish this. Upon discussion, using the household's maximum electrical draw was abandoned. The maximum power draw may have been too random for a realistic datum, i.e., it occurred during a power surge or blackout.

Instead, every household power draw, in $k W$ and at one minute intervals, was divided by the power draw representing its 95 th percentile. This was accomplished by creating a cumulative histogram of a single household over the entire study period, showing the frequency of the electrical draws divided into increasing bins. A cumulative histogram differs from a conventional one, because each bin is the frequency of occurrences in that bin's range, plus the summation of occurrences below that bin. In other words, if the frequencies are expressed as percentages of all the electrical draws, the final bin would have a value of one, because all the draws are either in this bin or below. An example of this is shown in figure 3.4, illustrating a household from the project. The 95 th percentile, the $k W$ value below which $95 \%$ of all the power draws would lie, is also shown in the figure. This percentile was selected over the maximum power draw, i.e., the 100th percentile, because it is a high power draw of the household that occurred repeatedly.

\section{Data division by day type and treatment of missing data}

The measured data was organized by day type, i.e., weekdays and weekends, of every season, i.e., winter, spring, summer and fall. This way, seasonal occupant driven trends could be noticed. The weekends were considered to be Saturdays and Sundays and the weekdays were from Monday to Friday. The beginning of spring, summer, fall and winter was considered to be March 21st, June 21st, September 21st and December 21st, respectively.

The data from the houses that were only measuring the overall, air-conditioning and furnace fan circuits needed to be downloaded every two months. The data from the houses measuring the additional appliances needed to be downloaded every month. During this downloading period of several minutes, the equipment did not log any data, creating a gap in the data sets. Rather than removing these days of useful 


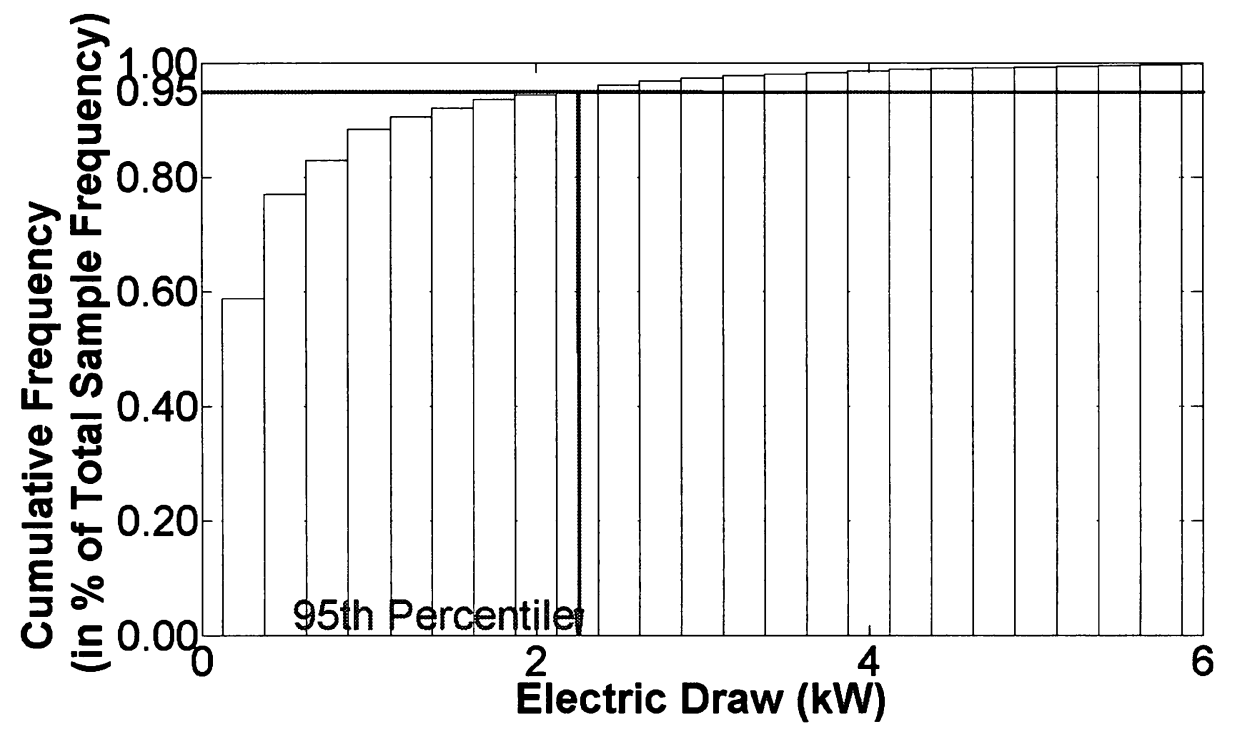

Figure 3.4: 95 th percentile illustration from a cumulative histogram from the data project.

data, the several missing minutes were replaced with the same minutes from another, randomly selected, similar day type, e.g. winter weekday.

Periods of malfunctioning equipment and/or maintenance also existed creating larger gaps in the data sets. In these instances, the affected days were removed from the data set for analysis.

\section{Measurement resolution and data smoothing}

Each CT, classified by its rating, had to be sized to the circuit that it was measuring. If the circuit was consuming enough electricity that the relay in the ET was constantly open, the CT rating was too small and could potentially miss consumed electricity. On the other hand, if a circuit was not consuming enough electricity for the rated size of the CT, it would not produce a magnetic field large enough to induce a current in the CT.

This introduced an issue for measuring circuits of devices that had large variations in power draws. This was seen in air-conditioners and furnace fans not in full operation. During the periods of low power draw, the logger would show several minutes of no power drawn by the device. Eventually, enough energy would be consumed by the device to open the relay in the ET, and it would seem that a sudden increase of power was drawn by the device in that minute. 
When subtracting the air-conditioners and furnace fans from the overall circuit to produce non-HVAC profiles, this resolution issue occasionally produced slightly negative electrical draws. These occurred when the overall household power draw was low, and the ET relays on the smaller devices were not opening in the same minute as the ET relay on the overall household.

To represent a more realistic power draw of devices in low power operation, the periods of coarse resolution were treated using a special algorithm. This algorithm searched through the original data sets, in terms of pulses, for minutes that recorded a value of zero followed by minutes with more zeros or a single minute with a value of one, to determine periods of coarse resolution. The number of minutes in a period that had a value of one occur was divided by the number of minutes in the entire period, to arrive at the average electricity drawn by the device. This is shown graphically in figure 3.5. This figure shows a furnace fan not in operation throughout the day except for a small period past 18:00. The furnace fan draws around $5 \mathrm{~W}$ after being "smoothed", compared to the original data which seemed to indicate that it sporadically drew $44 \mathrm{~W}$ in one minute after several minutes of drawing no power. This $5 \mathrm{~W}$ could be attributed to the furnace fan control system's quiescent power consumption which is the power drawn by the device when in standby. However, at this low of current the accuracy on the measurement makes this inconclusive. The purpose of the smoothing was to obtain realistic non-HVAC electric power draws when subtracting the air-conditioning and furnace power draws from the household's overall power draw.

\section{Data aggregation}

The data was aggregated in several different time-steps, namely: five, fifteen and one hour. This was done by averaging the power draws over the desired time-step. The purpose of this was for further validation with previously developed work, and to present the data, where needed, more concisely.

\subsection{Non-HVAC data results}

As discussed earlier, the household labelled $\mathrm{H} 8$ was removed from the analysis because their DHW was electrically heated and not monitored so their true non-HVAC loads 


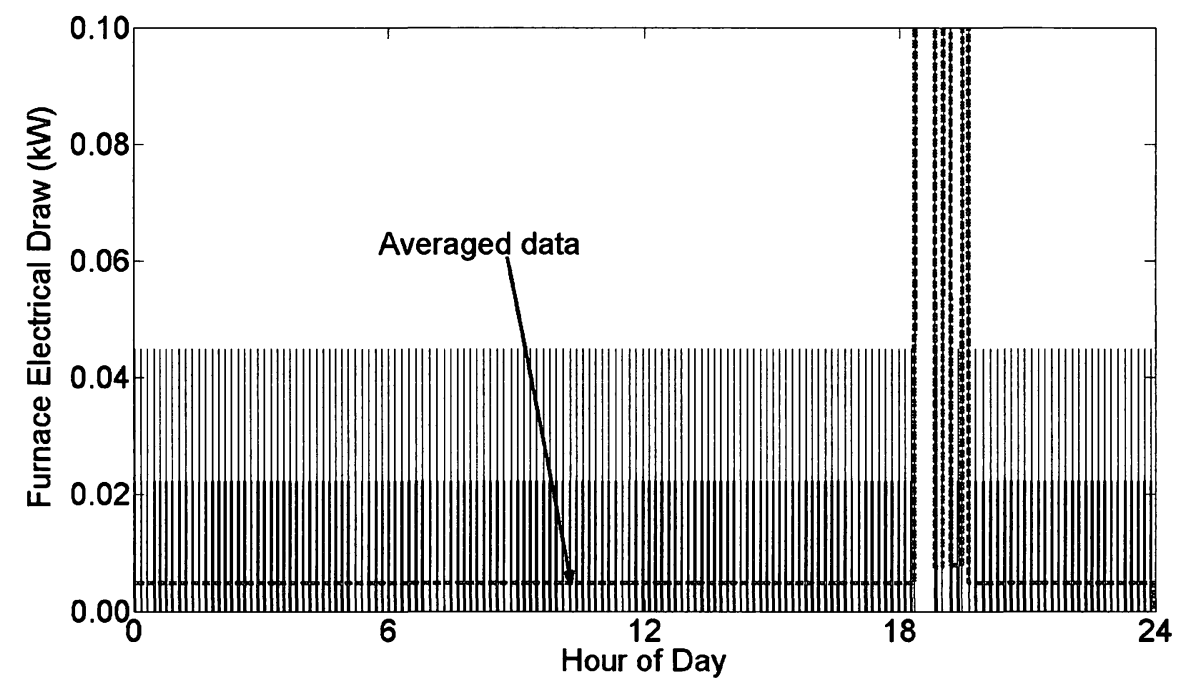

Figure 3.5: Example of data "smoothing" as a result of too coarse of a measuring resolution for a one minute logging interval.

were unknown. It should be noted again that at the time of analysis, a full year's worth of data was not available and the following lacks detailed data for more than a month beginning slightly after May 2010. All the data presented is in local time of the household, i.e., the time shown includes daylight savings time.

The 95th percentile non-HVAC electrical power draw of the households are summarized in table 3.4. These are compared with the 95th percentile non-HVAC electrical power draw of the synthetically generated profiles from Armstrong et. al [29] shown in table 3.5. It should be noted that the houses that are labelled in 3.4 are actual households in the Ottawa area and in no way relate to the synthetically generated profiles that are labelled in table 3.5.

Table 3.4 illustrates that the 95th percentiles of the households from this measurement project are not close to eachother. However, the generated profiles' values in table 3.5 are close to each other because they were derived using the same algorithm with the same target annual consumption. It should be noted that the houses from the measurement project in no way relate to the generated profiles.

The 95th percentiles from the houses monitored in the project do not increase with the increasing energy categories shown in table 3.4; e.g., some of the 95th percentiles of the houses in the low energy category are higher than the 95th percentiles in the 


\begin{tabular}{cc|cc|cc}
\hline House Label & Low $(\mathrm{kW})$ & House Label & Medium $(\mathrm{kW})$ & House Label & High $(\mathrm{kW})$ \\
\hline \hline H1 & 2.168 & H5 & 1.416 & H9 & 5.356 \\
\hline H2 & 0.945 & H6 & 1.791 & H10 & 2.8650 \\
\hline H3 & 1.567 & H7 & 1.327 & H11 & 2.3936 \\
\hline H4 & 2.388 & & & H12 & 3.435 \\
\hline
\end{tabular}

Table 3.4: 95th percentile non-HVAC electrical power draw of the households in the measurement project sorted by annual energy consumption level at one minute intervals.

\begin{tabular}{cc|cc|cc}
\hline House Label & Low $(\mathrm{kW})$ & House Label & Medium $(\mathrm{kW})$ & House Label & High $(\mathrm{kW})$ \\
\hline \hline S1 & 1.92 & $\mathrm{~S} 4$ & 2.96 & S7 & 4.67 \\
\hline S2 & 1.91 & S5 & 2.95 & S8 & 4.68 \\
\hline S3 & 1.93 & S6 & 2.92 & S9 & 4.67 \\
\hline
\end{tabular}

Table 3.5: 95th percentile non-HVAC electrical power draw of the synthetically generated profiles by Armstrong et. al [29] sorted by annual energy consumption level.

medium energy category. This indicates that houses reach the electric power draw representing their 95th percentiles in a manner that is not proportional to their total electricity consumption.

Figures 3.6 and 3.7 show households H12 and H10's electricity consumption on a randomly selected hot day, Aug 11,2009, respectively. The figures show the overall electricity power draw, labelled mains, the air-conditioner electricity power draw and the dryer electricity power draw. These two devices are the largest electricity consumers within the household.

These figures show the variance in daily electricity power draw between houses. H10 had a baseload two to three times larger than H12 over the day. Also, there is a significant increase of overall electricity draw in both households when the airconditioner and dryer are in operation. When both of these devices were in use, along with the other household appliances, the overall house electricity draw grew from a 


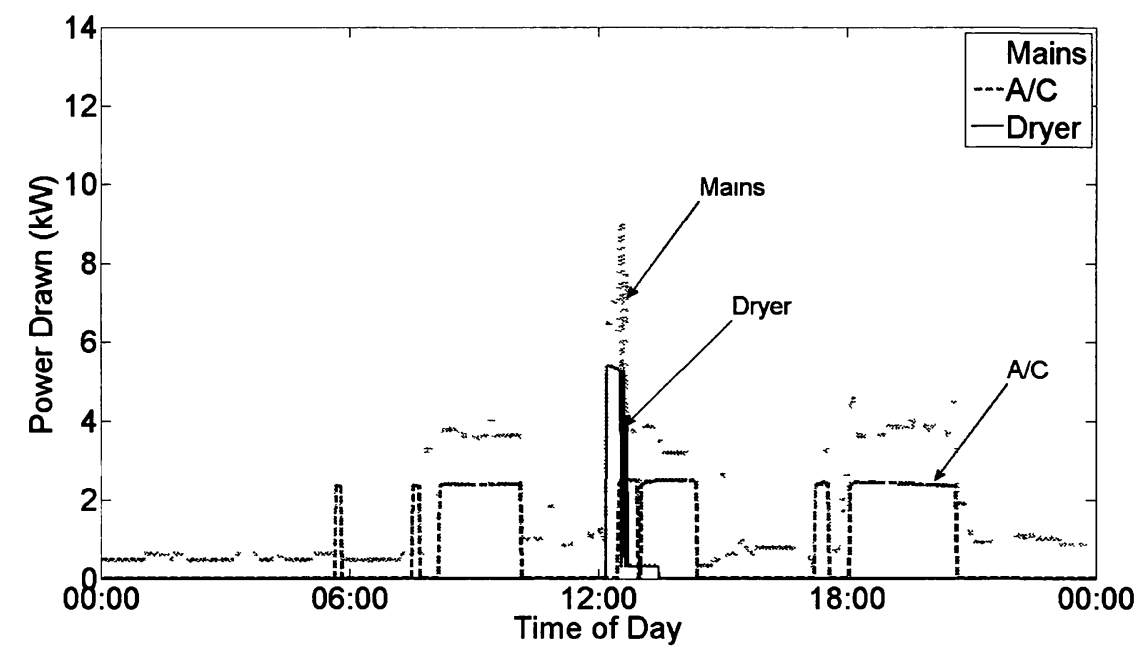

Figure 3.6: Household H12's electricity use at one minute intervals on Aug. 11, 2009.

baseload of approximately $0.7 \mathrm{~kW}$ to $9 \mathrm{~kW}$ in $\mathrm{H} 12$ and $1.8 \mathrm{~kW}$ to $13 \mathrm{~kW}$ in $\mathrm{H} 10$. These are clear examples of peak power electricity draw in residential houses.

Figure 3.8 show the seasonal variation of average electrical load between houses. Each plot shows the average daily non-HVAC electricity power draw of all the households in the project, separated by their energy category. These averages were produced by taking the data from every day in the season, including both weekends and weekdays. Overlayed on the summer and winter plots is the OPA's weekday TOU periods of low, medium and high electricity demand [9]. While the winter on-peaks coincide well with this sample population's household demand, the summer does not seem to. Note though that these plots do not include the space cooling electrical power draw which would increase household electricity consumption in the midday of the summer plot.

Figure 3.9 shows household H1's average daily non-HVAC electrical power draw over every summer weekday, plotted with two randomly selected summer weekdays. This figure illustrates the detail that would be lost in averaging multiple days of data. When averaged, the electricity peaks and lows tend to cancel each other out and produce a trend with little variation with respect to its previous time-step. The average curve's peak power draw does not exceed $3 \mathrm{~kW}$, whereas the daily maximum 


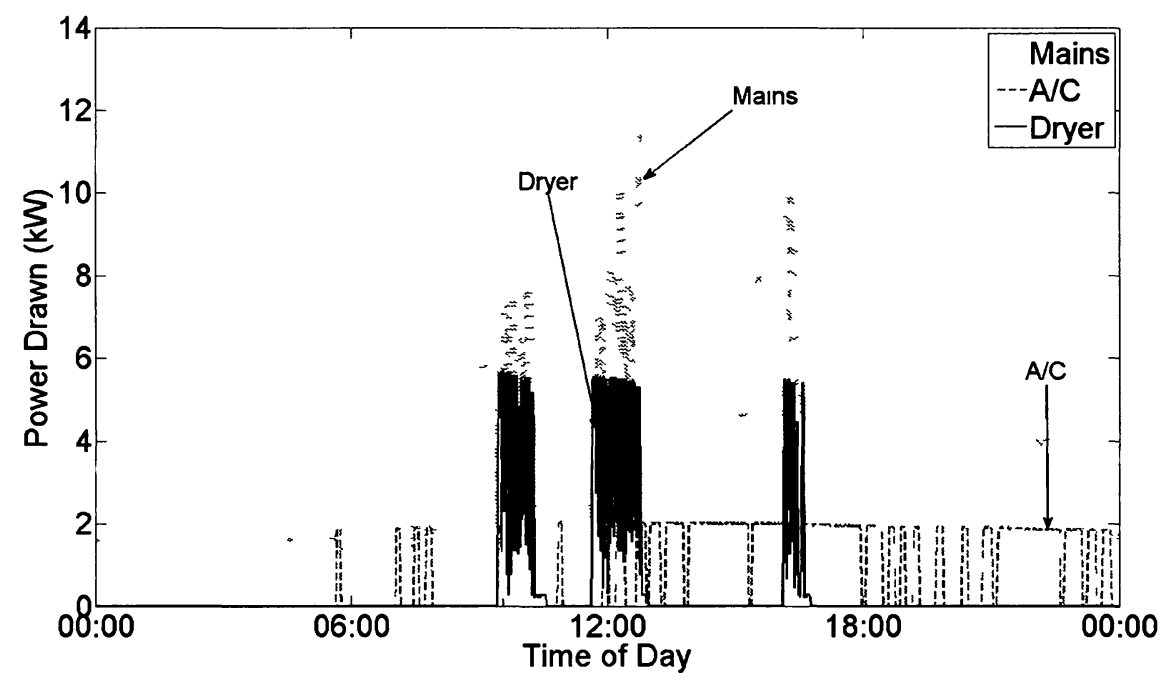

Figure 3.7: Household H10's electricity use at one minute intervals on Aug. 11, 2009.

peaks were more than $7 \mathrm{~kW}$ and $10 \mathrm{~kW}$ for days one and two, respectively. Averaging inherently defeats the purpose of analyzing peak residential loads and is not a good statistical peak demand estimator. However, averaging is effective in identifying trends of general energy consumption, i.e., baseloads and other periods for TOU schemes.

Plotting the standard deviation of electrical loads throughout the day may effectively illustrate the variation in residential loads. An illustration of this is shown in figure 3.10. This figure shows the daily household non-HVAC electricity power draw standard deviation using all the collected data for each house, separated by energy category. It illustrates that the variation of electrical loads of all the households is relatively low overnight and increases throughout the day, reaching a peak at around suppertime. The magnitude of the deviation scales by the energy consumption of the household, i.e., high, medium or low.

Another approach to analyze the distribution of electrical draws throughout the day may be to produce histograms. The data was aggregated to five minute intervals and normalized by the household's 95 th percentile as described in section 3.2.1. A normalized histogram of each five minute interval of the day, from all the collected data, would express the percentage of days that the household electric draw occurred 

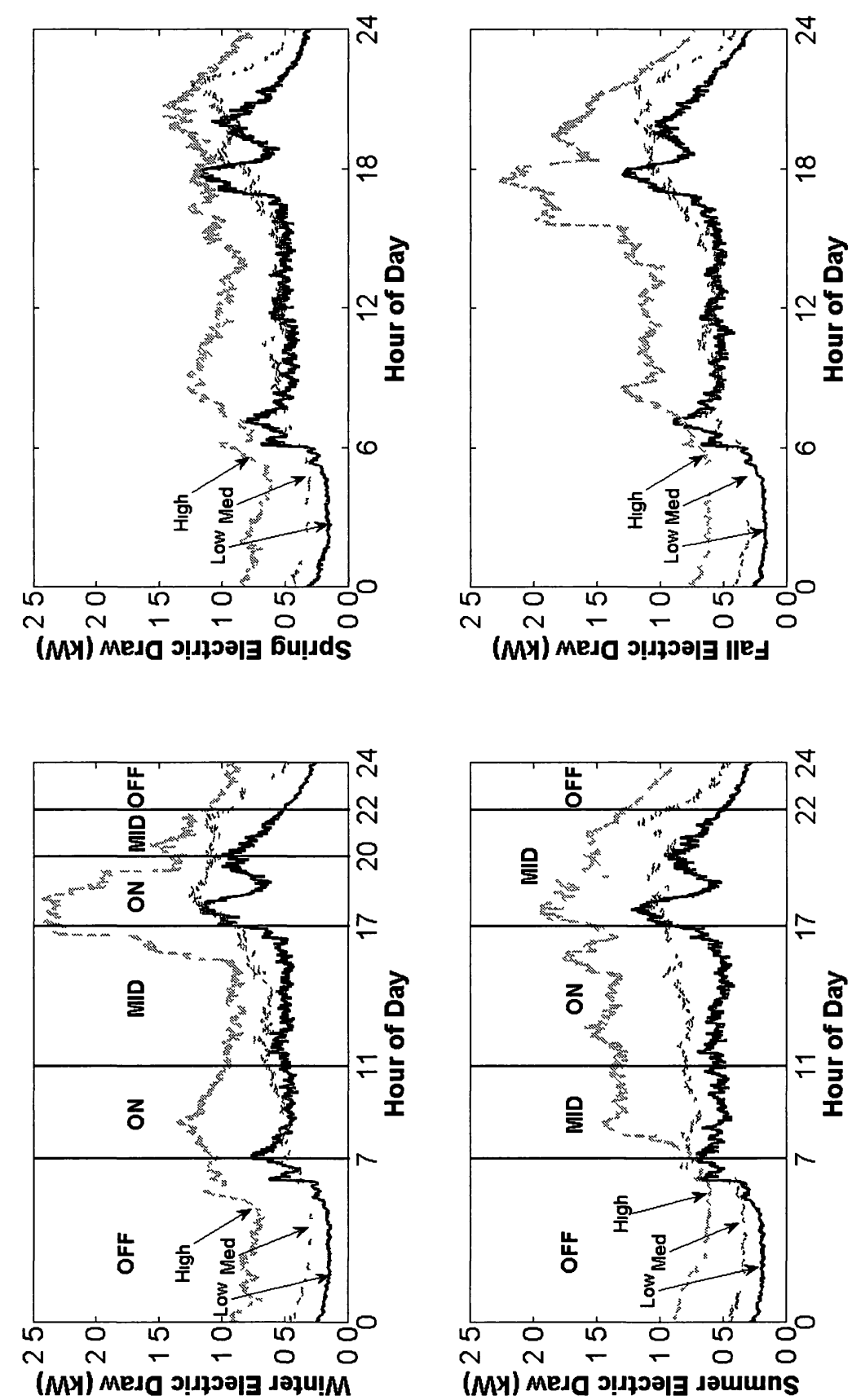

Figure 3.8: Daily average non-HVAC electricity power draw of all the households in the project, separated by their energy category, by season in $k W$ at one minute intervals. 


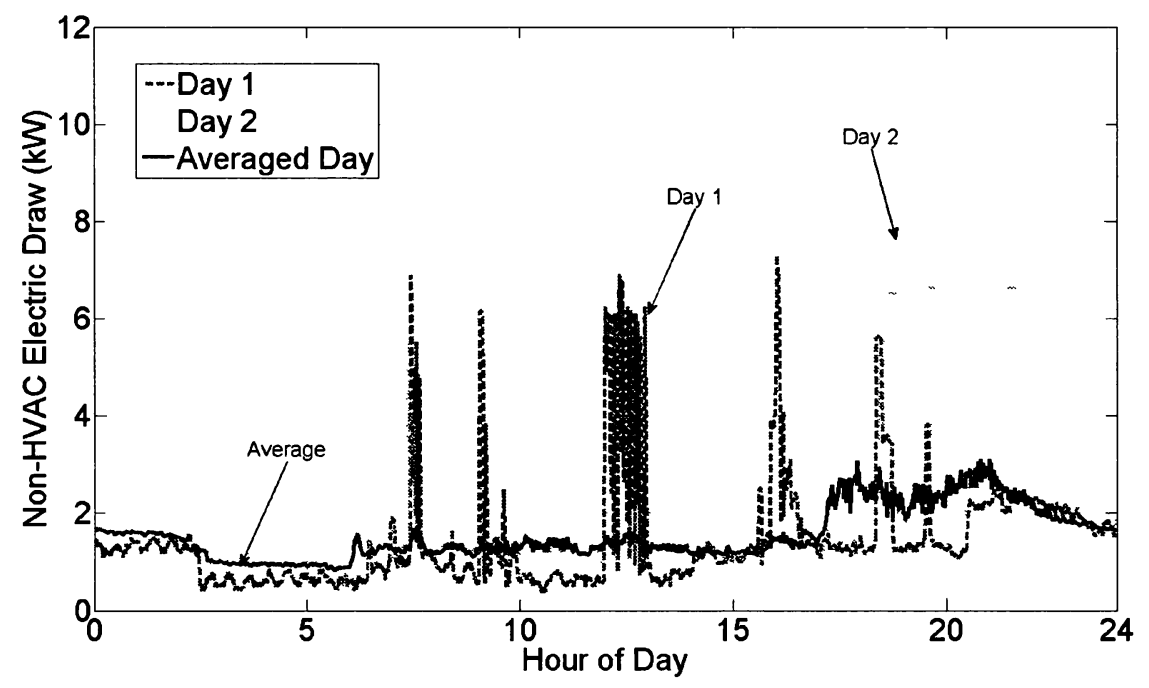

Figure 3.9: Household H1's average non-HVAC household daily electricity draw plotted with two randomly selected days.

in a given bin. This is also the probability distribution of the magnitude of electrical loads for that five minute interval. This is illustrated in figure 3.11 showing the normalized histogram of household H6's summer weekday non-HVAC electrical power draw between 0:00 and 0:05 in the morning. Referring to the figure, from the data collected for this household on a summer weekday between 0:00 and 0:05 in the morning, $19 \%$ of the days had a non-HVAC electrical draw occur around 0.1 or below of the household's 95 th percentile and $27 \%$ of the days had a non-HVAC electrical draw occur around 0.2 of the household's 95 th percentile, etc.

The normalized histogram of each five minute interval from all the collected data for one household was separated by bin value. A new plot was created showing each bin with 288 bars beside each other, one bar from each of the five minute interval histograms that corresponded to the bin. The 288 bars, making up an entire band for one bin, represents the number of days, in percentage, that the household drew the amount of electricity represented by that bin at every five minute interval.

A plot comparing the three medium households from the project compared to the three medium synthetically generated profiles from Armstrong et. al [29] using the normalized histogram approach above is shown in figure 3.12. The plot shows the distribution of household non-HVAC electrical power draws at any five minute interval 


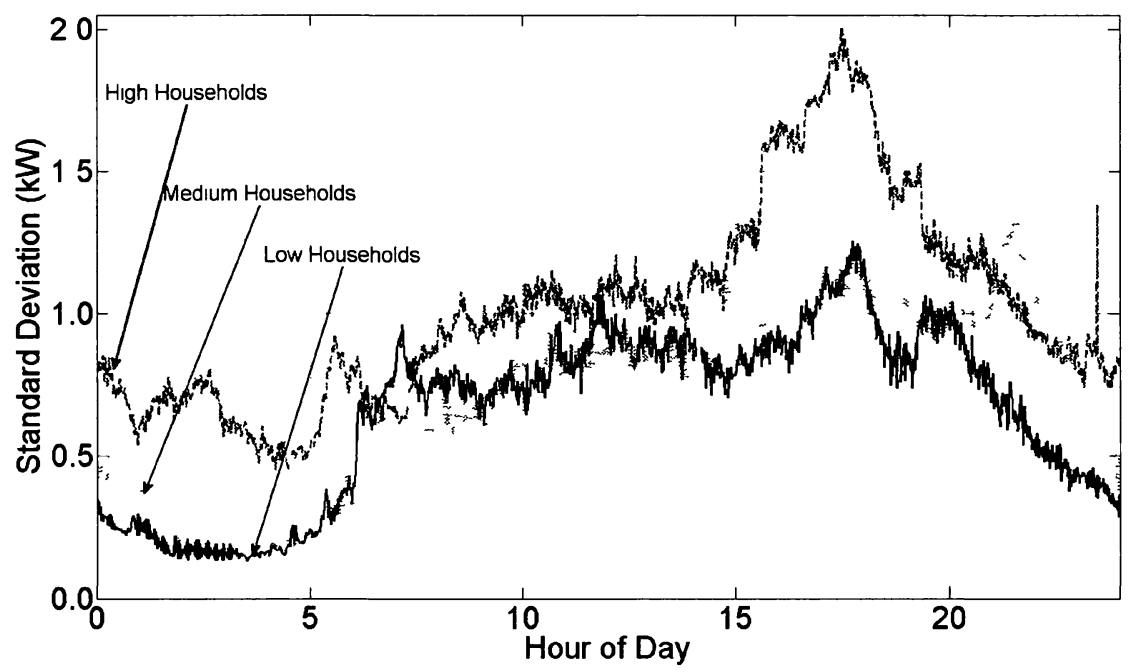

Figure 3.10: Daily household non-HVAC electricity power draw standard deviation using all the collected data for each house, separated by energy category.

on summer weekdays. The bins are fractions of the household's 95th percentile, ranging from 0.5 to 3.0. To clarify, for household $\mathrm{H} 5$ of the project between 0:00 and 0:05 in the morning (represented by the colour band on the top-left of the figure), $88 \%$ of the days had a non-HVAC electric draw occur around 0.5 and below of its 95th percentile, $10 \%$ of the days had a non-HVAC electric draw occur around 1.0 of its 95 th percentile and $1.2 \%$ of the days had a non-HVAC electric draw occur around 1.5 of its 95 th percentile, etc.

The bands provide an intuitive validation of household loads throughout the day. In the night time, a household typically has no appliances in operation, so the majority of household electric power draws at these five minute intervals will occur at low bins. In the morning, and throughout the day, non-HVAC activity increases, so the occurrence of electrical draws in the low bins decrease, and shift to higher bins. Over supper time, non-HVAC activity is at its highest and the occurrence of electrical draws is at higher fractions of the household's 95th percentile. In other words, the shape of the bands at the higher bins should be the inverse to the shape at the lower bins.

In comparing the data from the measurement project (shown on the top of figure 


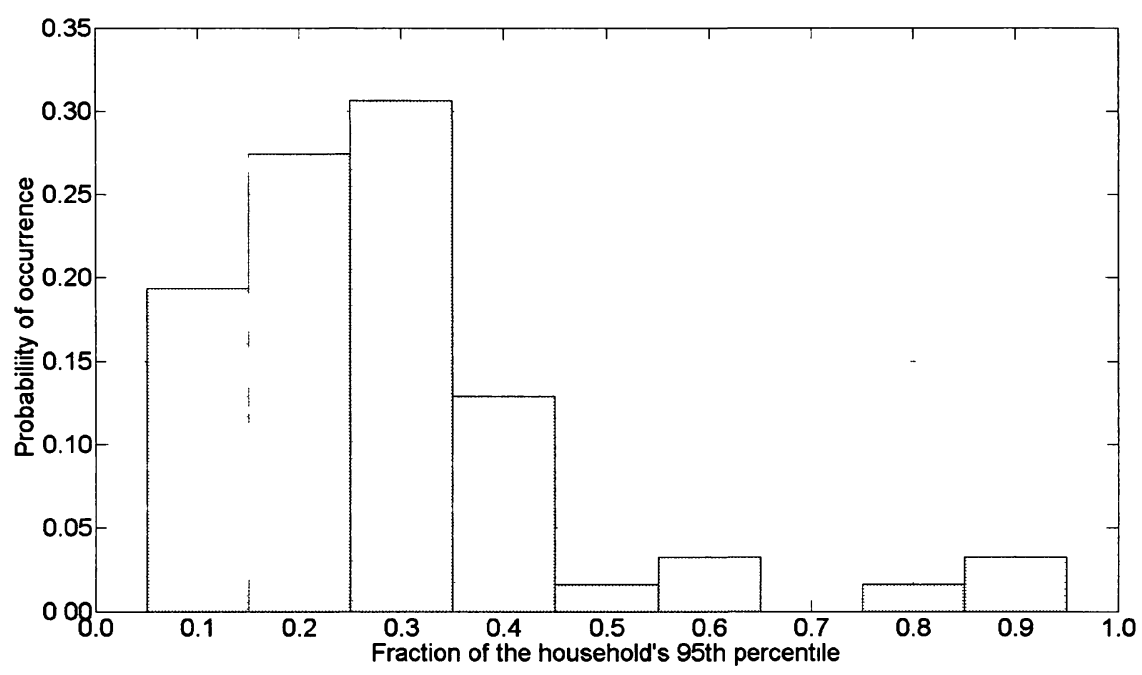

Figure 3.11: Probability distribution of household H6's summer weekday non-HVAC electrical power draw between 0:00 and 0:05 in the morning.

3.12) to the data from Armstrong et. al [29], the profiles seem to follow quite reasonably. The synthetically generated profiles show less daily variance of electrical draw occurrence between households, when compared to the houses from the measurement project; i.e., the bands from the generated profiles have the same general shape but the shape of the bands from the measurement project change between households.

Figure 3.13 shows the same comparison, but at higher values of the household's 95th percentile. Analyzing this graph shows much more occurrences of peak loads in the measured data than the synthetically generated data. This was up to eight times the 95th percentile in the measured houses. The synthetically generated profiles show no occurrences of electric power draw greater than three times the 95th percentile.

This indicates that while synthetically generated profiles may be an excellent costeffective representation of Canadian household non-HVAC electric loads, they lack the complexity necessary to capture the variation between households. This is inherent to algorithms that generalize random, and widely varying, occupant behaviour. This also indicates that the generated profiles do not exhibit the occurrences of high peak demand that is seen in actual households. A device simulated with the generated profiles to reduce peak demand would never need to satisfy the higher peaks that would be seen in the measured data. Business trips, vacations, relative visits, illness 


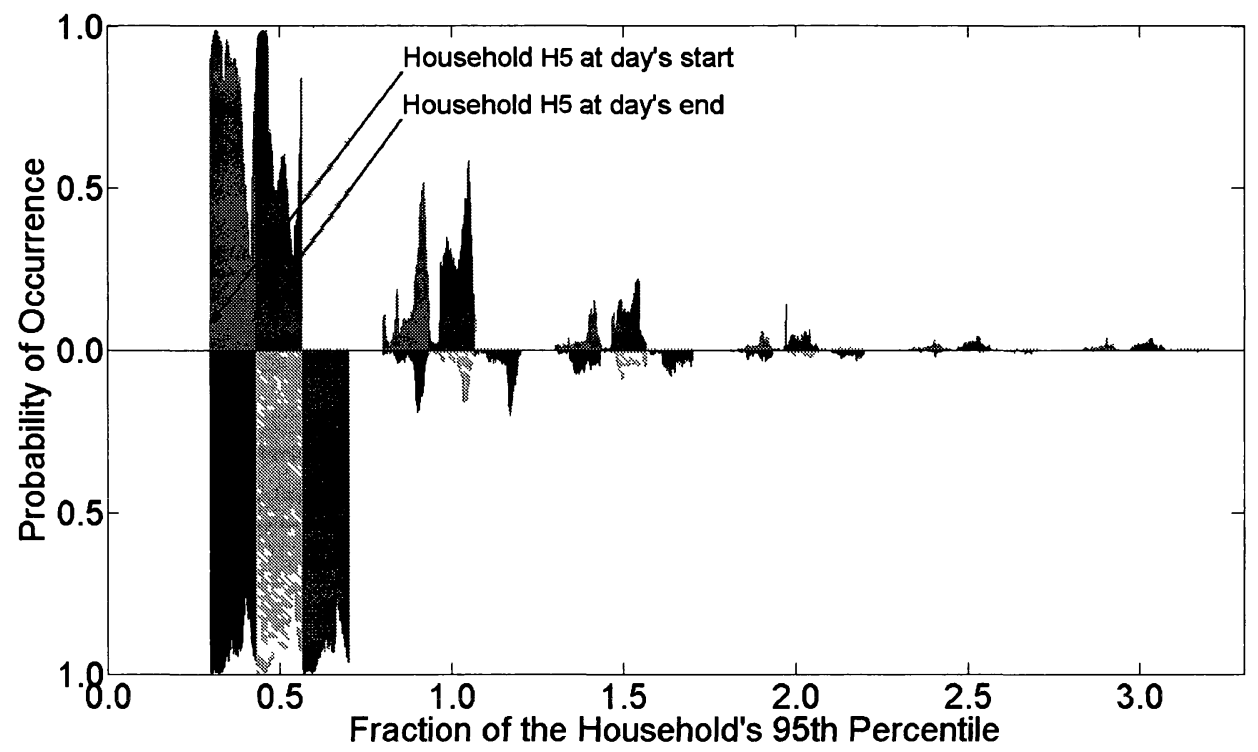

Figure 3.12: Comparison of normalized histograms of measured non-HVAC electric power draws of three medium houses in the measurement project with synthetically generated profiles from Armstrong et. al [29] over the year. The three bands from left to right on the top represent H5, H6 and H7 medium energy households from the project compared with the three bands from left to right on the bottom representing the three medium energy synthetically generated annual profiles from Armstrong et. al [29].

and weekend visits from children away at post secondary studies doing laundry, were just some of the events that the participants mentioned that deviated themselves from their regular routine. It is this complexity and randomness that make algorithms unable to reproduce occupant behaviour.

The work in this section analyzed and validated non-HVAC electrical usage data of Canadian houses at a fine time resolution. The non-HVAC profiles at one minute intervals can now be used in building simulation tools to represent household electricity usage. In the context of the research objectives, a micro-cogeneration device coupled with a li-ion battery can be assessed with greater validity. It can be simulated with several of the measured houses to asses its performance against the wide spectrum of Canadian residential electricity use. The data could be used for a new approach in representing non-HVAC residential electricity usage, explained in the future work section. 


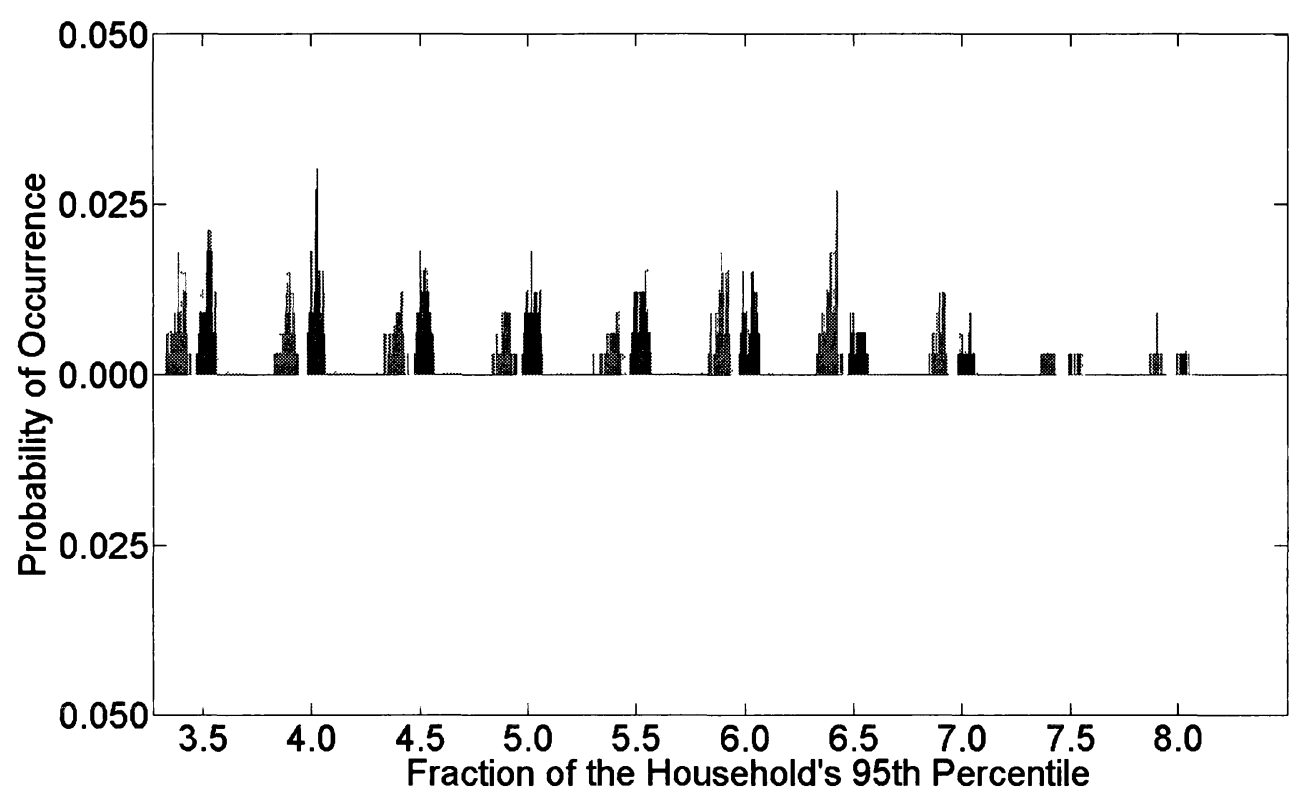

Figure 3.13: Comparison of normalized histograms of measured non-HVAC electric power draws of three medium houses in the measurement project with synthetically generated profiles from Armstrong et. al [29] at higher fractions of the household 95th percentile. The three bands from left to right on the top represent $\mathrm{H} 5, \mathrm{H} 6$ and $\mathrm{H} 7$ medium energy households from the project compared with the three bands from left to right on the bottom representing the three medium energy synthetically generated annual profiles from Armstrong et. al [29].

\subsection{Space cooling data analysis}

Space cooling, while technically an HVAC electrical load, shares many similarities with non-HVAC electric loads. Particularly, the use of air-conditioners is highly dependant on the habits and lifestyles of the household's occupants. As discussed in section 1.2.5, Beausoleil-Morrison [30] observed that occupant interventions to control the household temperature through openings and shading within the home have significant impacts on space cooling. Occupants can program their thermostats with different strategies throughout the day to alter these loads further. This concept is illustrated in figure 3.14, showing the air-conditioning electricity use over the day of three of the participants in the project on August 17th, 2009. These three households were chosen to show the difference in air-conditioning usage between households. 
The mean temperature in Ottawa that day was $25.6^{\circ} \mathrm{C}$ as reported by Environment Canada's Weather Office [j].

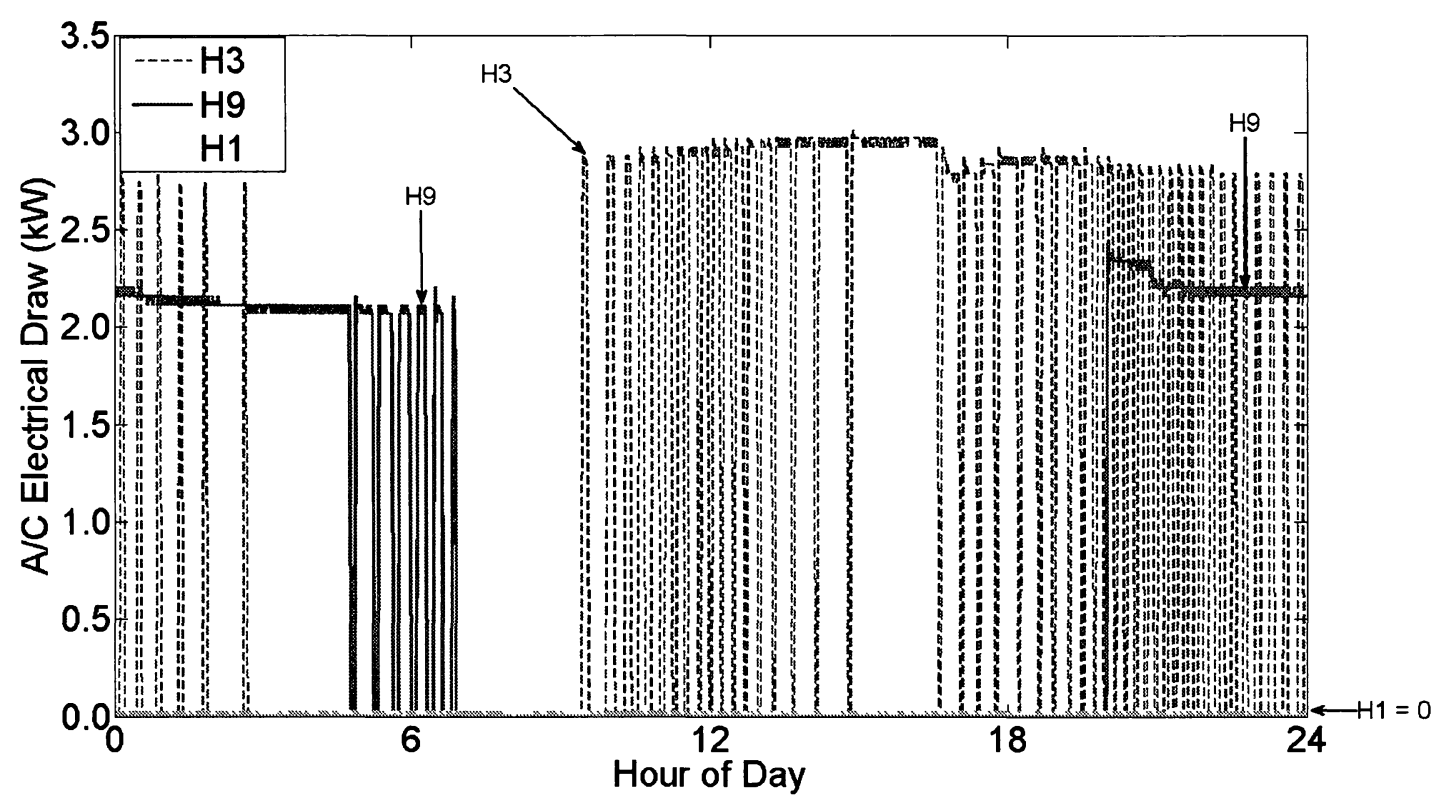

Figure 3.14: Example of air-conditioning variation between households on August 17 th, 2009 with a mean outdoor temperature of $25.6^{\circ} \mathrm{C}$ [5].

What this figure shows is that despite being subjected to the same conditions, all three houses were cooled in different ways. Household H3 was cooled throughout the day and overnight, $\mathrm{H} 9$ was only cooled overnight and $\mathrm{H} 1$ did not use their airconditioner this day at all. In this sense, space cooling resembles more of a non-HVAC load, in that it relies on the complexity of its users for its function and thus, is difficult to predict in building simulation. It is important to understand space cooling end use, beyond penetration rates of air-conditioners, in order to determine their impact on peak electric demand on the grid. By penetration rates, it would seem that all the three houses in figure 3.14 would contribute to the peak electric demand on that day. However, by collecting end user data, it is shown that each of the houses used their air-conditioners in unique ways.

Figure 3.15 shows the increase in household electricity consumption during the summer months due to air-conditioning of one of the houses in the project, H6. Each bar represents the overall electricity consumption (non-HVAC and space cooling), in $k W \cdot h$, in each month. The months of July and August see noticeable increases of 
total household electricity consumption. Note that some data from June was not available and these days were replaced with available data from around this time.

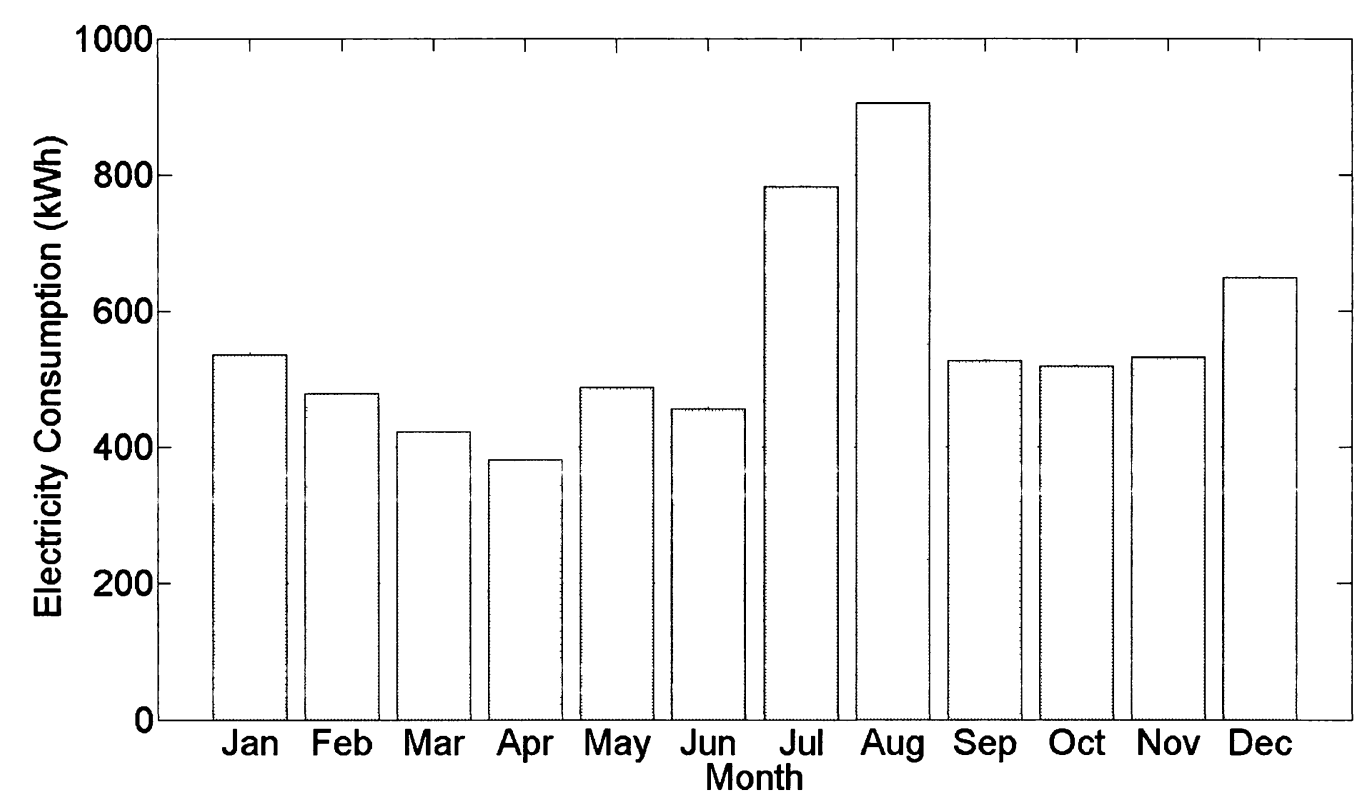

Figure 3.15: Example of monthly electricity consumption of one of the houses in the project, $\mathrm{H} 6$.

To explore the relationship between space cooling and outdoor temperature, scatter plots were generated from the collected data. The daily electricity consumed by each household's air-conditioner was plotted as a function of maximum daily temperature and mean daily temperature gathered from Environment Canada's Weather Office [5]. This plot is shown as figure 3.16. Note that the furnace circulation fan's electricity consumption was not included in this plot.

From this figure, there is a clear relationship between outdoor temperature and space cooling energy consumed over the day. At hourly temperature values over the day, this relationship would be lost. Figure 3.14 showed that occupants used their air-conditioners at different times throughout the day, sometimes even at night.

From the plot of air-conditioning consumption (see fig. 3.16), there is a difference in air-conditioning usage between energy categories. The low energy households tended to have lower daily space cooling electricity consumption than other households. In fact, it appears that even on some of the days with the highest maximum 


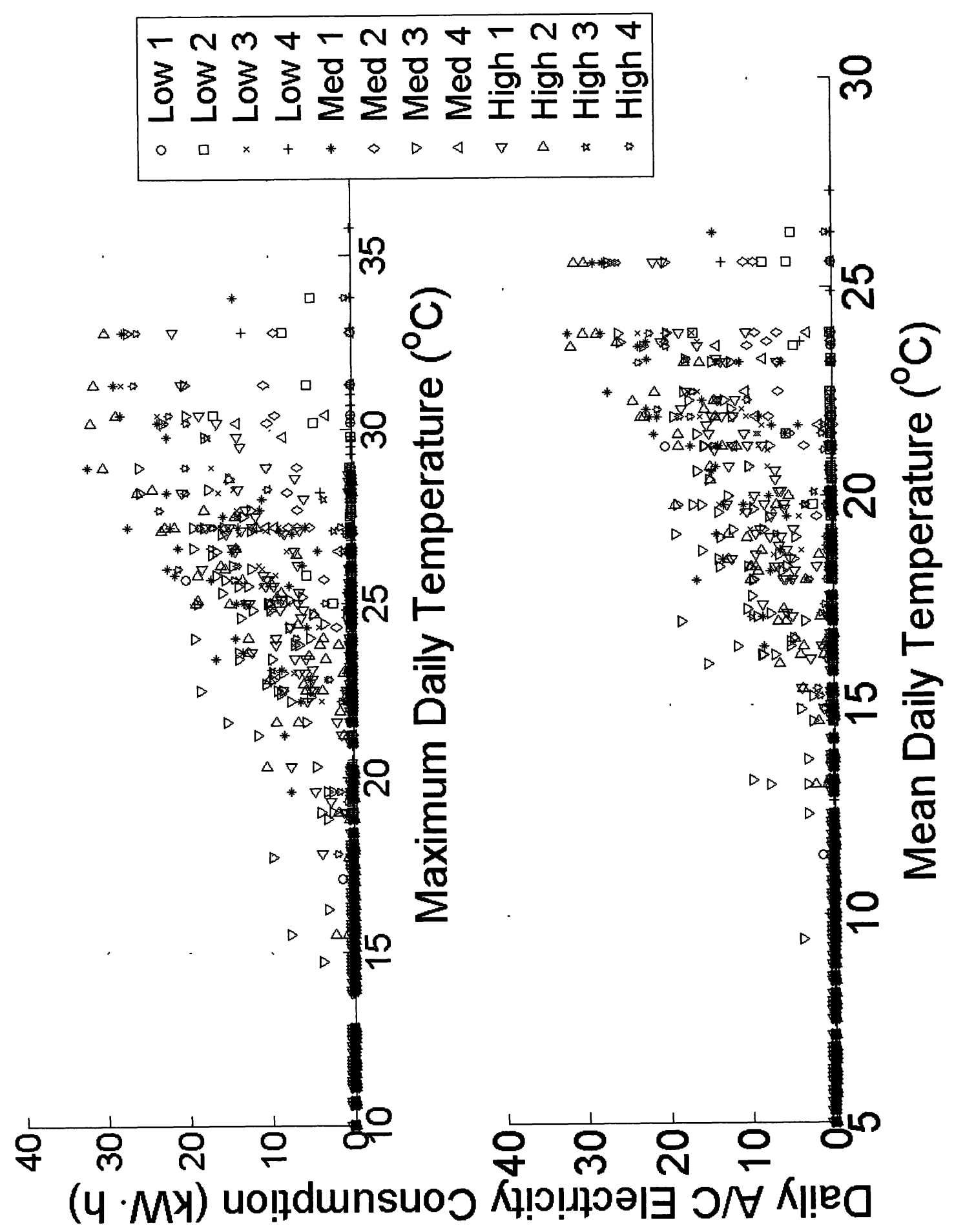

Figure 3.16: Household air-conditioning unit daily electrical consumption as a function of maximum and mean daily temperature. Note: Circulation fan electricity consumption is not included in this plot. 
daily temperatures, $\mathrm{H} 4$ resisted space cooling. This household compared to H10, for instance, shows significant differences in air-conditioning usage.

Building simulation tools, given proper inputs such as non-HVAC loads, should be able to accurately predict a household's heating and cooling demands; however as described, this becomes complicated in the cooling season with occupant mitigation via openings and shading. The household occupants in the project also filled out a brief survey of their willingness to open windows or use shadings to reduce their space cooling demand, as well as their thermostat setpoints throughout the seasons. The data collected and survey information provides excellent validation data to compare simulation results with. By incorporating probabilities and random number generation into building simulation to mimic occupant behaviour, space cooling prediction accuracy with building simulation may increase. This concept will be elaborated in the future work section.

The non-HVAC data collected in this project provides excellent input data for building simulation tools of Canadian residential electric loads. Using this data, HVAC systems designed to meet a household's energy demands can be more accurately assessed. The space cooling data represents real information of the wide spectrum of space cooling usage in Canadian residential homes during the summer. Using more sophisticated algorithms to mimic occupants' use of openings and shading may more accurately represent true occupant behaviour in building simulation. The space cooling data provides excellent validation for future work in this area. All the data collected in this project will be presented using Microsoft Excel sheets, providing specific details of each household and information about treatment of data, e.g., missing data, smoothing, etc. The gap of accurately representing residential electricity use in building simulation to assess a micro-cogeneration device with RES has been treated. The next research objective in this work, the development of a li-ion battery model in ESP-r, can proceed and is discussed in the following chapter. 


\section{Chapter 4}

\section{Lithium-ion battery model}

Following the adequate treatment of residential non-HVAC and space cooling electric loads in the previous chapter, a household can be successfully modelled in ESP-r. This model can now accurately simulate the thermal and electric demands of the household, and can assess an HVAC plant system designed to meet these demands in an efficient manner. This chapter discusses the next research objective of developing a li-ion battery model in ESP-r.

\subsection{Lithium-ion battery chemistry}

The chemical process in a li-ion battery is known as reduction oxidation (redox). Redox is a term given to any chemical process where atoms change oxidation states, i.e., they lose or gain an electron. This term covers many processes including all battery types, fuel cells, corrosion, etc.

The redox process in a li-ion battery involves the diffusion of lithium ions across an electrolyte when an external electric load is applied to its terminals [48]. These ions, originating from the anode with a high chemical potential, diffuse across the electrolyte towards the cathode with a low chemical potential. In doing so, an electron is passed not through the electrolyte, but via the external circuit to satisfy the imposed electric load. The diffusion process is driven by the difference in chemical potential between the anode and the cathode. This difference is the open circuit voltage (OCV) of the battery, or the maximum potential that the battery can deliver. It is the voltage measured across the terminal when no load is applied, i.e., when an open circuit exists (see eq. 4.1). 


$$
O C V=\mu_{\text {anode }}-\mu_{\text {cathode }}
$$

where $\mu_{\text {anode }}$ and $\mu_{\text {cathode }}$ are the chemical potentials of the anode and cathode, respectively, in Volts. The cathode of a li-ion battery is typically lithium intercalated with a metallic oxide. These metallic oxides include nickel, cobalt and manganese. The anode is typically a composition of lithium with carbon or graphite. During a discharge or charge, two half-reactions take place at the cathode and anode. For example, a li-ion battery with carbon at the anode and cobalt at the cathode would take on the following chemical half-reactions in discharge:

$$
\begin{gathered}
\mathrm{Li}_{0.45} \mathrm{C}_{6} \rightarrow 6 \mathrm{C}+0.45 \mathrm{Li}^{+}+0.45 e^{-} \\
\mathrm{Li}_{0.55} \mathrm{CoO}_{2}+0.45 \mathrm{Li}^{+}+0.45 e^{-} \rightarrow \mathrm{LiCoO}_{2}
\end{gathered}
$$

The electrolyte needs to facilitate the diffusion of ions, but be electrically insulated, as to not allow a short circuit or self-discharge. For $\mathrm{PbA}$ and $\mathrm{NiMH}$, the electrolyte has typically been an aqueous salt. However, due to the high reactivity of lithium to moisture, the use of an aqueous electrolyte is not possible. Hence the electrolyte is made up of a non-aqueous compound, typically lithium hexafluorophosphate, $L i P F_{6}$. Recent progress has investigated the use of a solid electrolyte polymer and the creation of a new battery type: the lithium polymer. The interested reader is referred to Dell [49] for more information on li-ion components and their evolution.

A large scale battery is made up of individual cells that are connected in series or parallel. Each cell has its own chemical potential difference and capacity, which is its available energy in $W \cdot h$. When cells are connected in series, their potentials are summed together, i.e., the overall voltage of the battery is the summation of the individual cell potentials; but the capacity remains that of a single cell. When cells are connected in parallel, the voltage remains the same as the lowest cell potential, but the capacity of the battery becomes the summation of each cell capacity. This introduces another consideration of cell balancing in the battery management system (BMS) of a li-ion battery. Each cell potential and capacity must stay balanced with the other cells in the pack. If one cell passes out of a safe operating range, the entire battery pack must be shut off by the BMS, losing the remaining available capacity. The BMS may continue operation, and risk damaging the cells that are out of safe 
operating range which could cause harm to the battery's environment.

State of charge (SOC) is the amount of capacity remaining in the battery, relative to a full battery. A SOC of $100 \%$ is thus a full battery. Depth of discharge (DOD) is the opposite of SOC, and the percentage of the battery capacity that has been discharged. The values of SOC and DOD describe the amount of lithium that has diffused from the anode to the cathode and depending on the manufacturer's definition, may not account for all of the lithium in the cells of a battery. Consequently, a cell, and hence a battery, may be able to be charged beyond a DOD of $0 \%$ (SOC of $100 \%$ ), and discharged beyond a DOD of $100 \%$ (SOC of $0 \%$ ). However, this may indicate unsafe ranges of operation to the manufacturer. Typically, the region of safe operation of a cell exists between a DOD of $0 \%$ to $80 \%$ [49].

As more lithium ions diffuse through the electrolyte from the half reactions in equations 4.2 and 4.3, the chemical potential rises in the anode and drops in the cathode causing the difference between the two to dwindle. At this point, the potential difference cannot drive the diffusion process; the cell's capacity has been exhausted and can no longer satisfy an imposed electric load. The OCV of a cell gradually decreases due to changing chemical potentials, making the OCV a good measurement of a cell's SOC. Typical OCV for a lithium ion cell begins at $4.0 \mathrm{~V}$, for a fully charged cell, and drops to $3.0 \mathrm{~V}$, for an empty one [49]. Any higher or lower and the cell risks being in dangerous operating ranges. These values vary slightly for different li-ion chemistries. A plot of the OCV as a function of DOD is important in the modelling of any battery chemistry, and each chemistry has a unique shape. A li-ion cell takes on the characteristic shape shown in figure 4.1. By accurately measuring the OCV of a battery, or its individual cells, it is possible to obtain its remaining capacity.

The rate at which the lithium ions diffuse is governed by the magnitude of the external load applied. The rate, in Ampere, that a cell will discharge at, is the power demanded, in Watts, divided by the cell potential, in Volts. Cells are typically charged and discharged at either constant power or current. To remain at a constant power during discharge, the current must be continuously increasing because the cell potential decreases as battery capacity decreases. Likewise, for a constant current discharge, the imposed load continuously decreases, directly proportional to the cell potential.

When the cell undergoes charging, an external power source is applied across the terminals, driving the lithium ions back from the cathode to the anode. Because by 


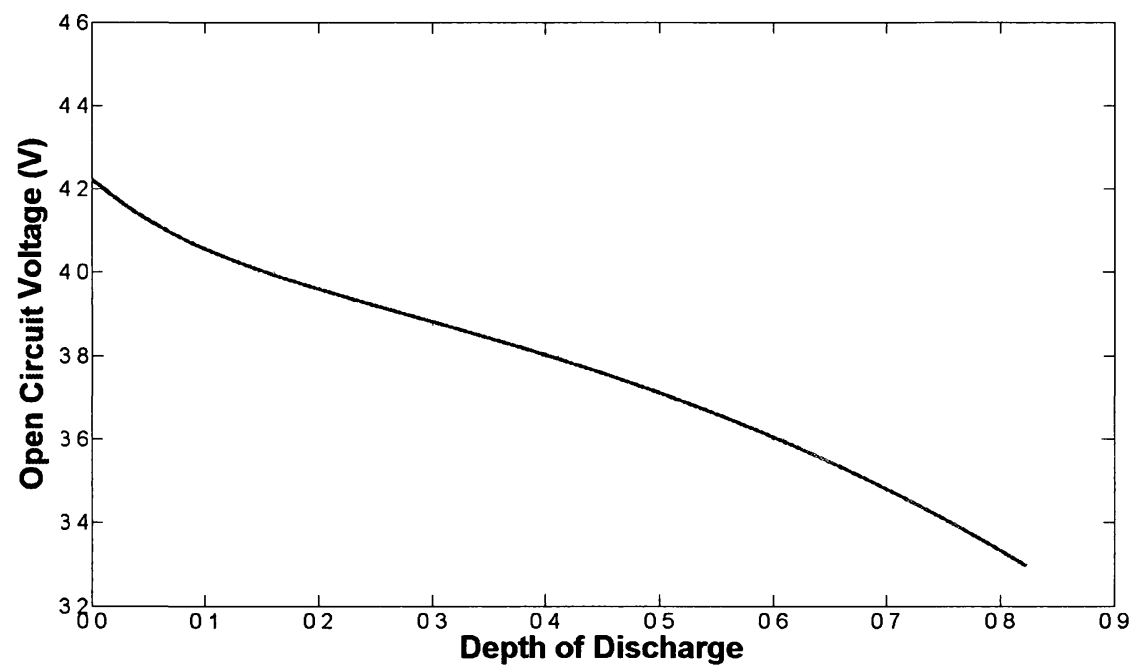

Figure 4.1: Open circuit voltage (OCV) as a function of DOD (1-SOC) for a lithium ion cell at $25^{\circ} \mathrm{C}$. [48]

definition, an anode is the component losing ions and electrons and a cathode is the one receiving, in a charging process the components switch and the anode becomes the cathode and vice versa.

The internal losses of a battery or cell constitute energy capacity lost during discharging, or excess energy needed during charging. They are affected by the rate of charge/discharge and the battery temperature [50]. Losses exist in two forms: electronic resistance and ionic resistance. Electronic resistance encompasses material resistances of the cell's metal components and terminal contact points. Ionic resistances occur due to complex reactions at the electrochemical level. They include electrolyte conductivity, ion mobility and electrode surface area [51]. Worth noting in electrolyte conductivity, is the formation of the solid-electrolyte interphase. This is the creation of a solid film on the surfaces of the electrodes in contact with the anode and cathode during the redox process. This represents a loss of original battery capacity and an increase in resistance [52].

While the OCV of a cell is the maximum voltage that could be delivered during discharge, the actual voltage that is delivered to the load is always lower and decreases linearly with current. These losses occur due to increases in kinetic reactions at the electrochemical level. The cell is limited to a maximum current that it can charge or discharge at without causing irreparable damage. The capacity of the cell also 
decreases with increasing current. That is to say, the voltage considered to indicate an empty cell, known as the cutoff voltage, will be reached with less total energy transferred to the load for a higher rate of discharge (i.e., a higher discharge current). This is briefly illustrated in figure 4.2 and will be elaborated upon further.

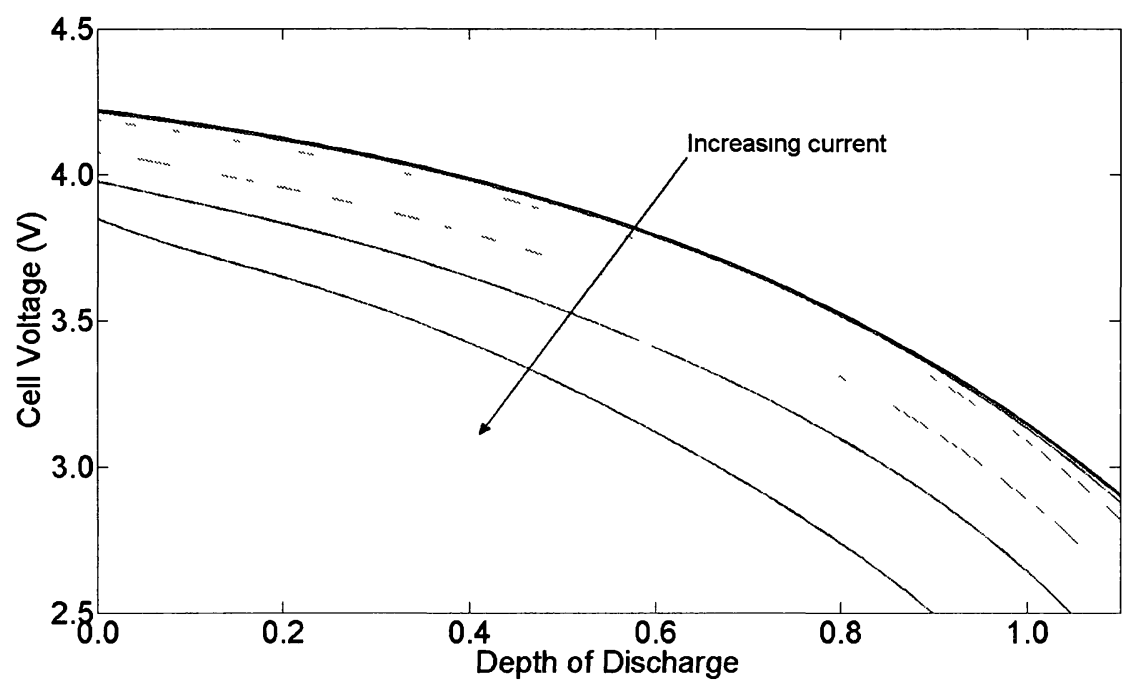

Figure 4.2: Voltage as a function of DOD (1-SOC) for a lithium ion cell with increasing current [48].

The voltage and capacity of a cell are also dependant on the temperature of the cell. Thus, the OCV curve as a function of DOD is given at a reference temperature, typically $25^{\circ} \mathrm{C}$. As temperature increases in a cell, the lithium ions can diffuse with less kinetic interference, and the internal resistance decreases. Capacity also increases with increasing temperature for the same argument as current. The opposite effect on voltage and capacity is seen for a lower cell temperature. This is illustrated in figure 4.3 .

Self-discharge is the amount of charge lost from chemical reactions and diffusion of lithium ions during storage time without any external load applied. While these values have typically been low in li-ion batteries, 2 to 8 percent per month [20], efforts have been made to quantify this. Some [53] [54] propose that the self-discharge of a cell, $S_{D}$ in Ampere, can be expressed using an Arrhenius equation (see eq. 4.4). 


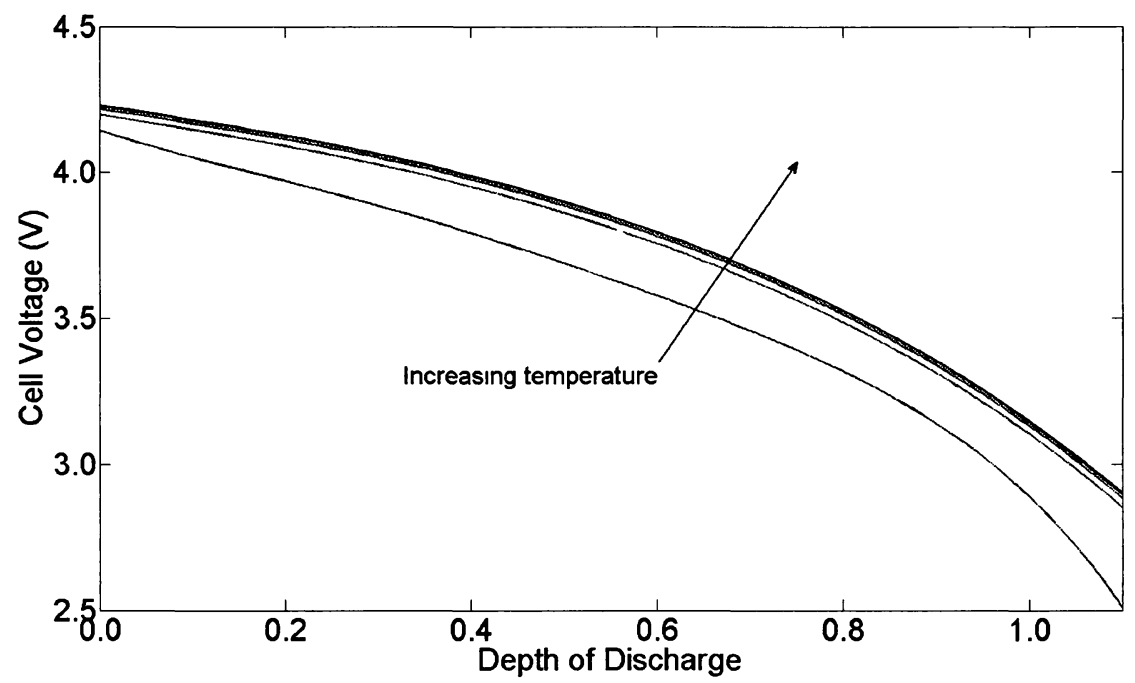

Figure 4.3: Voltage as a function of DOD (1-SOC) for a lithium ion cell with increasing temperature [48].

$$
S_{D}=k_{0} e^{\frac{-E_{A S}}{R_{g} T}}
$$

where $E_{A S}$ is the activation energy of the reaction in $k J \cdot k m o l^{-1}, R_{g}$ is the gas constant in $k J \cdot \mathrm{kmol}^{-1} \mathrm{~K}^{-1}, T$ is the temperature of the cell in Kelvin and $k_{0}$ is a constant in Ampere.

Battery degradation due to cycling is the loss of initial capacity over time with repeated charging and discharging. This is a complex quantity to define that depends on how far the battery has been discharged, how far the battery has been recharged, the rate at which this is done and the battery temperature [54]. Spotnitz [55] describes this loosely using a diagram of battery capacity as a function of the number of cycles used, shown as the left plot in figure 4.4.

In this figure, battery capacity decreases sharply over the beginning of the battery's life (region A) and tapers off as the number of cycles increase until the end of region $\mathrm{C}$. At this point, the battery's capacity has been reduced to about $80 \%$ of its original, and the battery is considered to be at the end of its operable life. After this point, the capacity will decrease at an increasing rate (region D). Spotnitz [55] states that data on region $\mathrm{D}$ is hard to find as manufacturers tend to suppress this information. This relationship between capacity and cycle life can be linearly approximated 


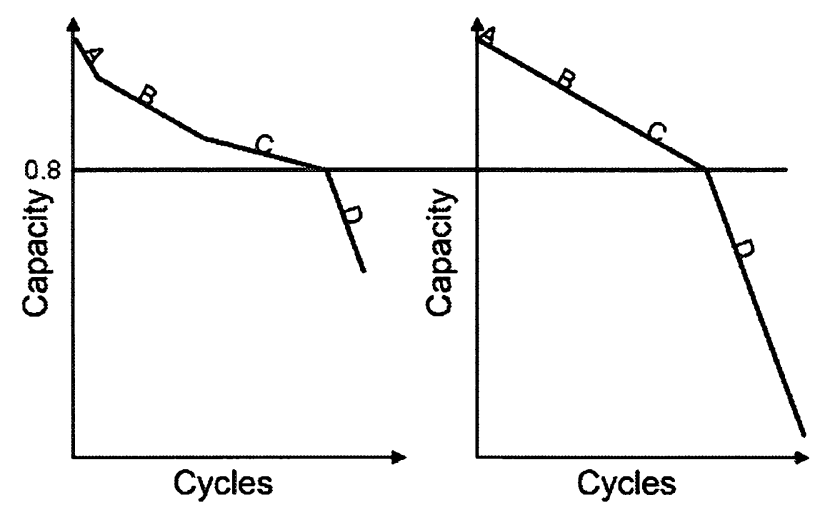

Figure 4.4: Diagram of remaining battery capacity as a function of cycle life as described by Spotnitz [55] (left) and approach used later in li-ion model (right).

as shown in the second plot of figure 4.4. This will be revisited in later chapters.

The development of an accurate li-ion battery model requires the modelling of all the points discussed above. That is, the ability to capture internal resistance as a function of current and temperature, self-discharge of a battery and long term battery degradation while keeping the battery balanced and in safe operating limits.

\subsection{Lithium-ion battery modelling}

\section{Finite difference approach}

There are several different approaches to battery modelling, each having their own benefits and limitations. The finite difference approach is the highest level of resolution. This approach involves the discretization of the anode, cathode and electrolyte of a li-ion cell. By applying the laws of conservation of mass, momentum and energy, the diffusion of lithium ions through these discretized nodes, driven by the chemical potential gradient between the anode and cathode, is modelled. The model can be specified in all spatial coordinates and unsteady temporal frames. This approach is closely based on Fick's law of diffusion [56]:

$$
\frac{\dot{m}_{A}}{A}=-\vec{D} \cdot \nabla C_{A}
$$

where $\frac{\dot{m}_{A}}{A}$ is the mass flow rate with respect to time per area, $D$ is a proportionality diffusion coefficient in area per time, and $C_{A}$ is a mass concentration per volume. 
The largest advantage to this approach is the ability to accurately capture the true behaviour of a battery's internal resistance, essentially its efficiency, by applying different boundary conditions. This includes the relationship of resistance with current and temperature, other phenomena such as impurities in the manufacturing process, which may lead to ion concentrations on the electrode surfaces, and realistic long-term battery degradation. Limitations to this approach are the same experienced in conventional computational fluid dynamics. These include the truncation of higher order terms in Taylor series expansion, etc. Another limitation is the lack of empirical data, specifically in terms of life cycle degradation, to apply realistic boundary conditions to the model. A drawback limiting an implementation of such a model in this work is the integration of this solver with the rest of a whole building simulation program such as ESP-r [25]. Examples of this modelling approach include that of Darcovich [48] and the VRFB work by Ribberink and Wang [26].

\section{Equivalent resistive network approach}

The equivalent resistive network approach is the next level of resolution for battery modelling. This model uses a lumped capacitance approach with state parameters such as DOD and OCV. It characterizes the internal resistance of a battery which, neglecting manufacturing impurities, is a function of current and temperature. Typically, this results in the use of a resistor-capacitor ( $\mathrm{RC}$ ) network to capture the real internal resistance component, from constant current draw, and the imaginary impedance component, from changes in current rates with respect to time, of a battery (see fig. 4.5). This relationship between resistance, current and temperature, while non-linear, has been approached in several ways. Some use polynomial fits to empirical data of charge and discharge curves [57]. This method tends to neglect the complexity of the resistance and still requires significant data and effort to extract the polynomial coefficients. Others attempt to quantify the value of the equivalent $\mathrm{RC}$ network using Nyquist plots $[53,58]$. The limitations to the equivalent resistive network model is the lumped capacitance assumption. This assumption does not capture realistic occurrences such as battery degradation due to ion concentrations from impurities in the manufacturing process, and long-term reduction in battery capacity from cycling; although work has been done to include this phenomena as well [26]. Its advantage in the context of this work is that its use of state functions 


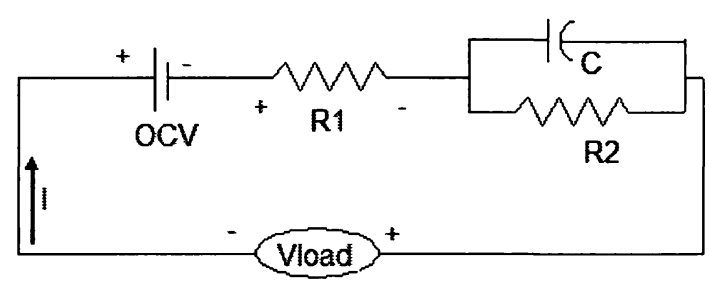

Figure 4.5: $\mathrm{RC}$ network schematic used in lumped capacitance modelling of li-ion battery.

can be integrated within ESP-r's power flow network (see section 2.2) with ease.

\section{Gao approach}

Recent work by Gao et al. [50], has introduced a new approach based on a lumped capacitance assumption and state variables. This approach uses a reference curve, based on a polynomial curve fit to empirical data, to determine the battery's DOD as a function of OCV. Rather than attempting to derive relationships between current and internal resistance, and temperature and internal resistance, this approach simply scales the reference curve by factors depending on the battery's current and temperature. These factors are measured empirically and are unique to each battery, making this approach potentially general to all battery types. Validation by Gao et al. [50] has shown adequate accuracy using this technique; however, verification and calibration would be necessary in the implementation of such a model.

\section{Approach analysis}

In analyzing the different battery modelling approaches above, the approach by Gao et al. [50] seems most suitable for an application within ESP-r. The finite difference approach may be the most realistic in capturing true battery behaviour; however, it would require the definition of several quantities and boundary conditions on the electrochemical level. A model using this approach for a VRFB has been somewhat implemented in ESP-r [26].

The second approach of lumped capacitance parameters is the basis of the approach used by Gao et al. [50]. Where the Gao approach differs is in capturing the relationship between internal resistance, current and temperature. In the lumped 
capacitance approach, the internal resistance and battery capacity is calculated using long empirically-fit polynomials, with coefficients relating to both current and temperature. Extracting the coefficients to properly calibrate a battery would be difficult. Calibrating a model using the Gao approach would only require a set of charge/discharge curves at various currents and temperatures. By this argument, the Gao approach will be used for this work's development of a li-ion battery model.

\subsection{Li-ion modelling approach for present work}

The model chosen for this work is based on a lumped-capacitance approach to evaluate the true voltage of the battery, given an applied electric load based on the current and temperature. This approach involves the use of an $\mathrm{RC}$ network as shown in figure 4.5 .

In an RC network, a portion of the OCV is dropped across a real resistor (labeled $R 1$ in figure 4.5), and a complex resistor/capacitor (labeled $R 2$ and $C$ in figure 4.5) before arriving at the load. The OCV as a function of DOD is expressed as a $5^{\text {th }}$ order polynomial fit to measured data, at a reference temperature:

$$
O C V(D O D)=\sum_{\imath=1}^{5} E_{\imath} D O D^{\imath}
$$

The real resistor accounts for the resistance due to the magnitude of current and increases linearly with current. The voltage during discharging is then:

$$
V(t, D O D, I(t))=O C V(D O D)-I(t) R_{\imath n t}(D O D)
$$

where the $O C V$ is measured in Volts, $I(t)$ is the current in Ampere and $R_{\imath n t}$ is the internal resistance in $\Omega$. This results in a vertical shift of the voltage as a function of DOD curve, equal to the internal resistance multiplied by the current, as illustrated in figure 4.6.

An RC network is able to capture the impedance of any device, real or complex, by equating the device's real impedance into a resistive load and its complex impedance into a resistor/capacitor load. The resistor/capacitor impedance accounts for internal resistances due to changes in current with respect to time. In general, this impedance captures losses in batteries in a continuous time frame from highly transient currents 


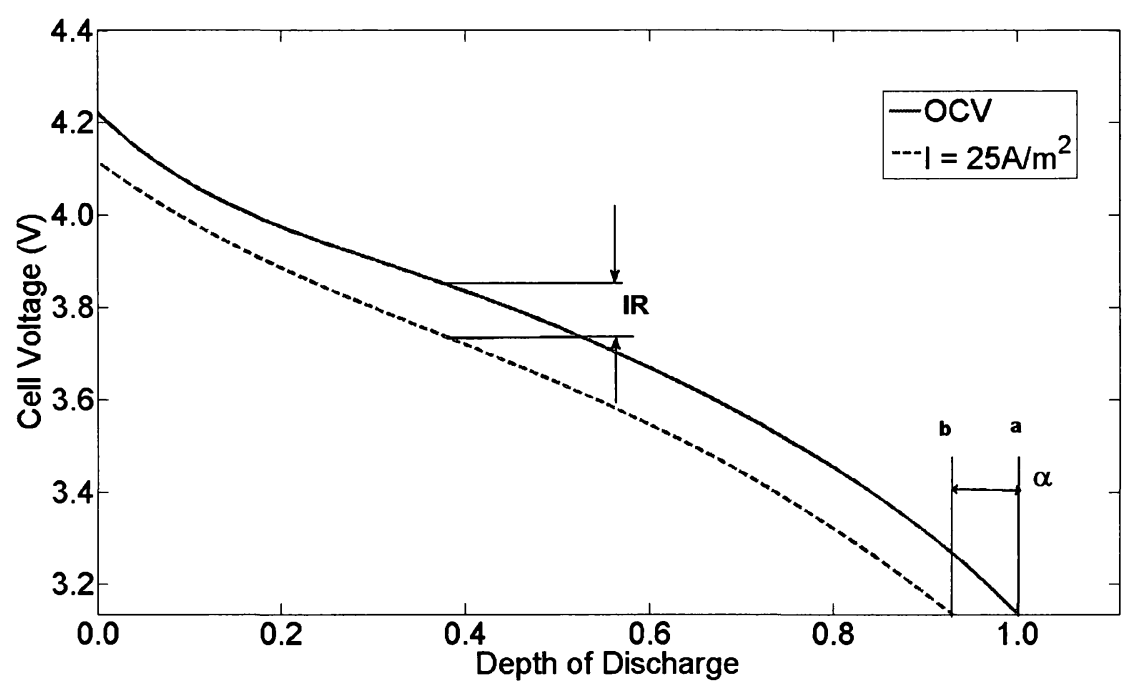

Figure 4.6: Transformation of voltage as a function of DOD curve due to an imposed current.

imposed. This is significant in electric vehicle applications, for example, where "pulse" current or power might be required from the battery on the magnitude of seconds or less. Simulations in ESP-r occur at discrete time-steps in a quasi-steady-state fashion within the time interval. The battery current during the time-step is considered uniform and steps instantly with respect to the next time-step. Although, in reality this is false because the load is continuously changing. As such, the impedance term in figure 4.5 is removed in this model formulation. Future work should include exploration into the complex impedance contribution from a li-ion battery under changing current with respect to time, if any exists.

Battery capacity also decreases with increasing current. Where other approaches try to capture this capacity change using another polynomial fit, Gao et al. [50] treats this by measuring the difference in battery capacity between the OCV curve and the voltage curve representing the desired current. This is done at the same reference temperature and is illustrated in figure 4.6. The OCV battery capacity, labelled "a" in figure 4.6 , is the reference capacity, in $A \cdot h$ (if the voltage is assumed to be nominal over the entire discharge). The capacity for the curve at the higher current is labeled "b". Gao et al. [50] defines a current factor, $\alpha$, as the ratio of these values.

$$
\alpha=\frac{b}{a}
$$


Battery temperature plays a similar role as current in the transformation of OCV with respect to DOD. For a charge or discharge at a battery temperature different than the reference, the voltage at the load will be different than the OCV. Gao et al. [50] defines this simply as a $\Delta V(T)$ (see fig. 4.7).

$$
V(t, T)=O C V(D O D)+\Delta V(T)
$$

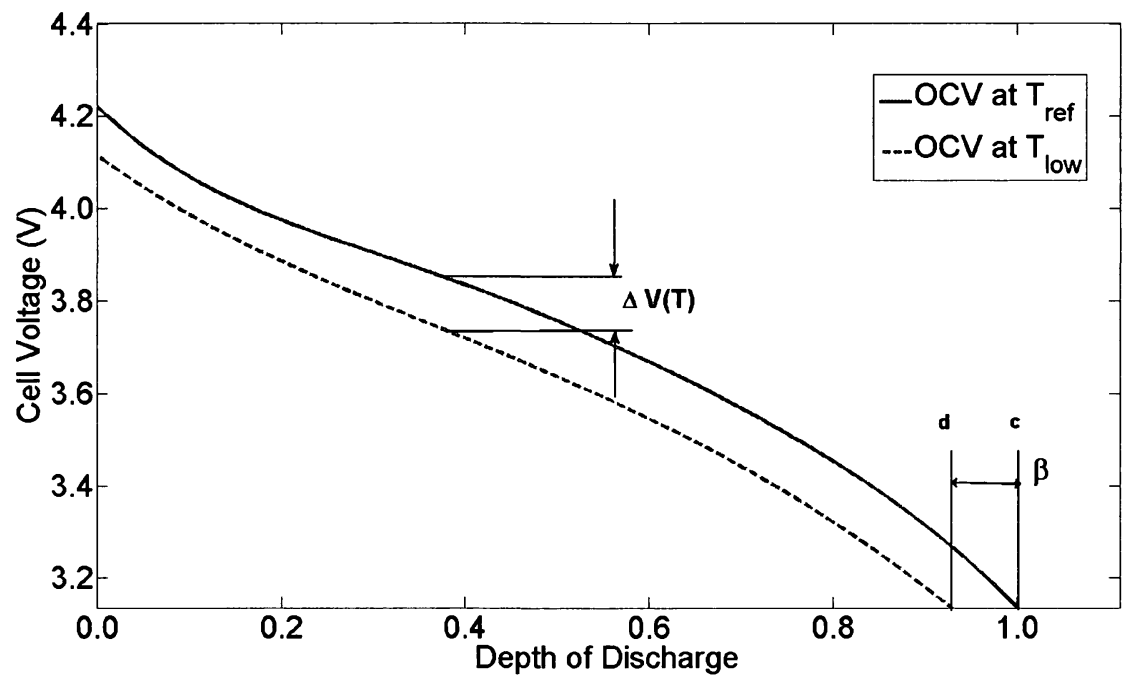

Figure 4.7: Transformation of voltage as a function of DOD curve due to a change in temperature.

The battery capacity also increases or decreases with increasing or decreasing temperature from the reference temperature, respectively. Gao et al. [50] defines this as a temperature factor, $\beta$, which is the ratio of the battery capacity at its reference temperature, "c", and the capacity at the battery's actual temperature, "d".

$$
\beta=\frac{d}{c}
$$

The change in DOD of a cell is the change, in percentage, of its capacity. Thus, the DOD at the end of a time-step of a cell discharging at constant current, taking into account effects of current and temperature is then:

$$
D O D_{\text {end }}=D O D_{\text {rnttaal }}-\frac{I \Delta t}{\alpha \beta C a p_{\text {ref }}}
$$


where $C a p_{\text {ref }}$ is the reference capacity of a single cell in $A \cdot s, I$ is the discharge current in Ampere, and $\Delta t$ is the time-step in $s$. In charging mode, the second term on the right side of equation 4.11 would be positive. The cell's capacity, $C a p_{\text {ref }}$ will also vary with the amount of cycles the cell has undergone, as previously discussed. This will occur in a linear fashion as shown in the second plot of figure 4.4. At the end of every time-step, the model will take the absolute value of the change in DOD, $\triangle D O D$ and add this to the number of cycles already spent. This will be elaborated further.

The final temperature of the battery at the end of a time-step is found by applying a transient conservation of thermal energy over the battery:

$$
m C_{p} \frac{d T}{d t}=I^{2} R-h A\left(T-T_{a m b}\right)
$$

where $m$ is the battery's mass in $k g, C_{p}$ is the battery's specific heat in $J \cdot k g^{-1}$. $K^{-1}, h A$ is a heat transfer coefficient, including convection and long wave radiation exchange with the battery's environment, times the battery surface area in $W \cdot{ }^{\circ} C^{-1}$, $T$ and $T_{a m b}$ are the battery temperature and ambient room temperature, respectively, in $K$, and $I^{2} R$ is the internally generated heat of the battery which is equal to the square of the current times the internal resistance, in Watts. This method assumes a lumped capacitance approach and that conduction heat transfer is insignificant compared to convection and radiation.

The first order ordinary differential equation shown in equation 4.12 , with the boundary condition $T(t)=T_{\text {int }}$, has the following unique solution:

$$
T_{f}=\left(\frac{I^{2} R_{\imath n t}}{h A}+T_{a m b}\right)\left(1-e^{-\frac{h A}{m C_{p}} \Delta t}\right)+T_{\imath n \imath t} e^{-\frac{h A}{m C_{p}} \Delta t}
$$

With approaches to model the electrical and thermal performance of a battery as shown above, a li-ion model can be implemented into ESP-r. By having data sets representing charge/discharge curves at various currents and temperatures, values for $\alpha, \beta, \Delta V(T)$ and $R_{\imath n t}$ can be extracted to calibrate the model.

\subsection{Implementation in ESP-r battery subroutine}

As mentioned, an electric storage model existed in ESP-r prior to the current work. It was developed in a subroutine that is capable of having additional storage models 
appended to it. $\mathrm{A} \mathrm{PbA}$ model, using a lumped-capacitance $\mathrm{RC}$ approach, and a VRFB, using a basic finite difference approach, exists in this subroutine [26]. The li-ion battery model developed in this work, using the approach by Gao et al. [50], is appended to this subroutine. At each time-step, the subroutine must calculate if the li-ion battery will inject or draw electricity into the electrical network node where it is defined, or remain idle.

To determine the battery's contribution, the subroutine begins the simulation by initializing monitoring flags to zero and retrieving the battery's initial state values. The monitoring flags developed in this work for the li-ion model are over/under voltage flags and over/under temperature flags. These flags are part of the li-ion BMS to ensure that the voltages and temperatures of the battery remain within safe limits. The initial state values for the li-ion model are the battery's temperature and DOD. At subsequent time-steps, the monitoring flags are re-assessed. If the temperature and/or voltage is higher or lower than the allowed operating range, the appropriate monitoring flag is turned on. These flags are revisited later in the subroutine.

Next, the subroutine retrieves the power demanded from the battery, assigned from outside the subroutine. If this value is positive, by convention, power is required from the battery, and it is placed in discharge mode. If the power demanded is negative or zero, the battery is placed in charge or idle mode, respectively. In charge mode, the power demanded is made positive for the remainder of the subroutine. Where and how the power demanded to the battery is assigned will be discussed in chapter 6 .

The maximum current that the battery can deliver or receive over the next timestep is then calculated. This uses an existing subroutine in ESP-r [26]. This current is limited by the minimum allowable DOD (or voltage) in charge mode, and the maximum allowable DOD (or voltage) in discharge mode. The subroutine calculates the current that would reach these charge/discharge limits using the following:

$$
\begin{aligned}
& V_{\text {max }}=V_{\text {end }}+\left(R_{\imath n t} I\right) \\
& V_{\text {min }}=V_{\text {end }}-\left(R_{\imath n t} I\right)
\end{aligned}
$$

where $V_{\max }$ and $V_{\min }$ are the upper and lower cutoff voltages of the battery and $V_{\text {end }}$ is the final voltage of the battery at the end of the time-step. The end voltages and internal resistance of the battery are dependant on the current and the final DOD at 
the end of the time-step. These values for the PbA and VRFB models are calculated using specific approaches in existing subroutines. New subroutines, calculating these values using the approach by Gao et al. [50] explained above, have been added to include the function of a li-ion battery. These subroutines are continuously accessed throughout the model's function at different points. Other subroutines to calculate values such as final current, final power, final temperature and internal heat have also been previously developed for the other storage models. They are also accessed throughout the main subroutine. Once again, new subroutines performing these same functions, but for the li-ion modelling in this work, have been developed.

Returning to the maximum current subroutine, because the final voltage and internal resistance are dependant on the current, the subroutine uses an iteration technique to converge to a final solution. After the maximum current for this timestep is determined, the corresponding maximum power is found. Again this takes into account internal resistance and temperature effects in its calculations. The maximum current and power are limits on the battery's capabilities for the time-step. The interested reader is referred to the paper by Ribberink and Wang [26] on how this occurs in more detail.

The li-ion model then enters its BMS subroutine, developed in this work, to control the battery, and revisit its flags. Given the present state of the battery (i.e., DOD and temperature), the external power demanded or received, and the maximum power/current available, the BMS assesses the appropriate action to take over the next time-step. This is explained in the BMS flowchart show in figure 4.8.

If the over/under voltage flags were set, the model calculates the amount of power, in Watts, needed to be discharged/charged in the next time-step in order to return the battery into a safe range using the following equation:

$$
\text { NeededPower }=\triangle D O D \frac{C a p V_{a v g}}{\Delta t}
$$

where $\triangle D O D$ is the change in DOD that the battery must go through to return to safe limits. The battery capacity (accounting for current and temperature), Cap in $A \cdot s$, and the voltage over the time-step, $V_{\text {avg }}$, is a function of current which is initially unknown. Thus, the current is initialized to zero and the process iterates until convergence.

Once the power needed to discharge/charge the battery back to its safe limits has 
been found, the BMS subroutine decides from where it will come. From figure 4.8, if the battery mode coincides with what is necessary to return to its safe limits, e.g., if the battery is already in discharge mode and an over voltage flag is set, the routine will see if the demanded power can be met. For example, if $100 \mathrm{~W}$ is demanded from the battery, and it needs to discharge $50 \mathrm{~W}$ to return within a safe range, it can easily do both. If it cannot still satisfy the demand, excess electricity will be needed for this time-step. Any surplus or deficit of electricity needed over a time-step will be met by the electric grid. This will be discussed in further detail in chapter 6 .

If the battery mode does not coincide with what is necessary to return the battery to safe limits, e.g., if the battery is already in discharge mode and an under voltage flag is set, the battery mode is changed from discharge to charge. In this case, the maximum current and power requirements are re-assessed for charging the battery over the next time-step rather than discharge. The battery will be assigned, in this case, to charge at the necessary power without exceeding the maximum current and power limits. This case will always result in an unbalance between the battery power demanded and the battery power delivered, ultimately relying on the grid to return to safe limits.

In the case of an over temperature, the battery will be assigned to idle over the time-step. This continues for subsequent time-steps until enough heat has dissipated from the battery to its surroundings, using equation 4.13 , to bring it back into a safe temperature range.

In the case of an under temperature, the battery is equipped with an electric resistance heater. While this function is based on the work by Ribberink and Wang [26], it has been modified and appended in this work. The BMS will divert a portion of its charge to heat the battery to a safe operating range. The amount of power available for heating the battery over the time-step, in Watts, is:

$$
\text { AvailableHeat }=\frac{V_{a v g}^{2}}{R_{\text {battery }}}
$$

where $V_{\text {avg }}$ is the average battery voltage over the time-step and $R_{\text {battery }}$ is the battery's heater resistance in $\Omega$. The power required to heat the battery to a safe temperature, in Watts, is found by re-arranging equation 1.13:

$$
\text { NeededHeat }=\left(\frac{T_{m i n}-T_{\text {init }} e^{-\frac{h A}{m C_{p}} \Delta T}}{\left[1-e^{-\frac{h A}{m C_{p}} \Delta T}\right]}-T_{a m b}\right) h A-I^{2} R
$$


The routine then assigns power to heat the battery over the next time-step as long as it does not exceed the maximum available power based on the battery voltage and heater resistors (eq. 4.17). If the needed heat is less than the originally demanded power, the difference between will still be devoted to charging or discharging the battery, and the electric grid will make up any deficit. If the needed heat is greater, the battery load will be set to zero and the demand will be satisfied by the electric grid.

If none of the battery safety flags have been turned on, the battery will enter its normal operation as shown in figure 4.8. The battery will be assigned to discharge or charge, depending on the battery mode, to meet the demanded power. This will be limited to the maximum current and power discussed earlier. If the battery is in idle, the routine calculates the self-discharge using equation 4.4.

The BMS subroutine completes and returns the state values of battery DOD, final temperature, current, voltage and internal heat to the main subroutine. It also calculates the amount of cycles used in this time-step, by taking the absolute difference between the final DOD and the beginning DOD. This is to account for battery degradation, as explained above, which will be elaborated in more detail in the following chapter. If the battery was assigned to charge over the time-step by the BMS, the power and current is made negative to follow convention. The main subroutine records the values in an XML output, and writes the voltage and phase angle (for the case of a DC current this is zero) to the power flow node where the battery is assigned.

For a user to successfully make use of the li-ion battery model in ESP-r, a proper calibration file and control file must be created and implemented. These are necessary to properly define the parameters of a specific li-ion battery and to assess how and where power is sent to the battery with respect to the entire household plant system. Chapters 5 and 6 outlines this procedure. 


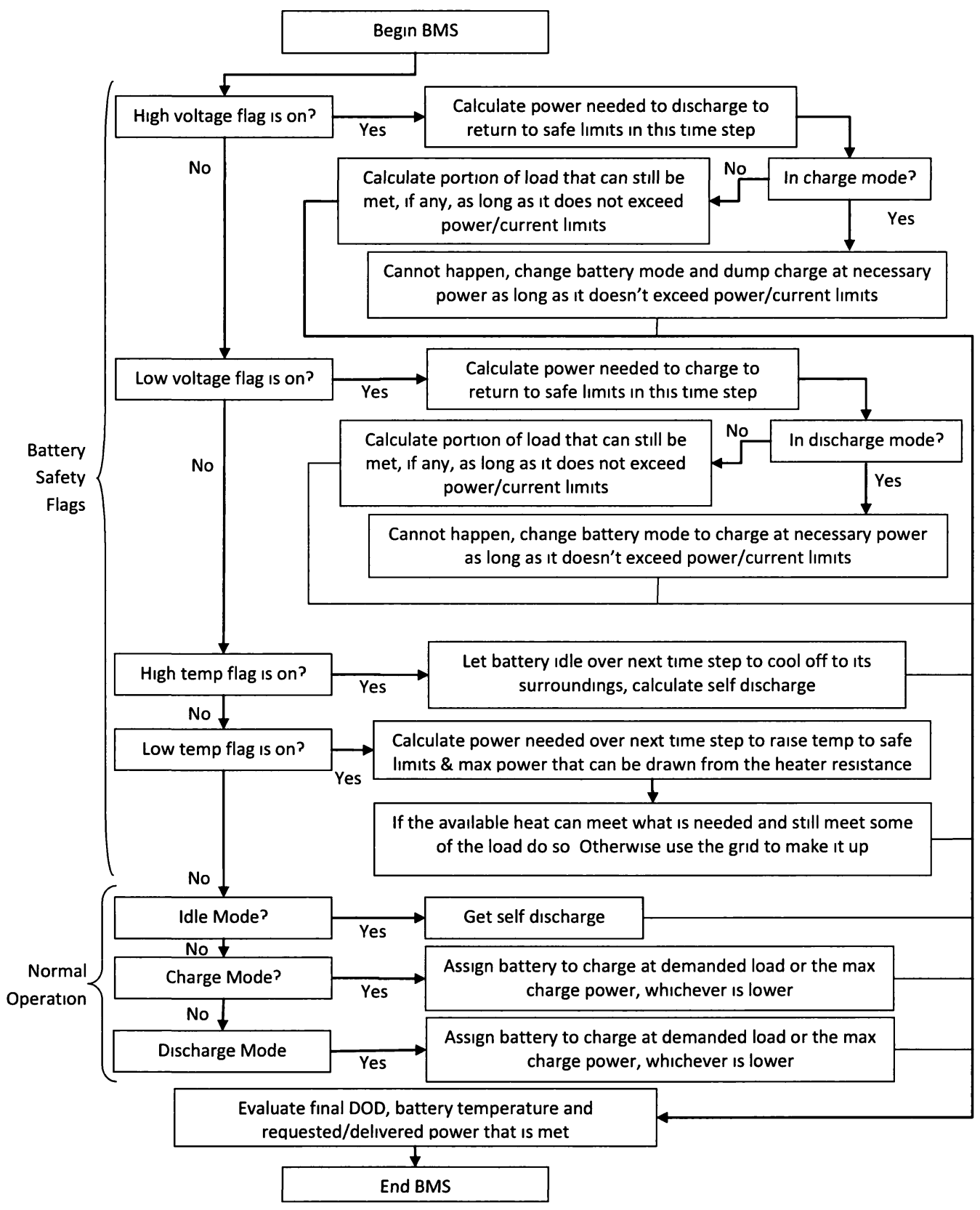

Figure 4.8: Battery management system used in li-ion battery subroutine. 


\section{Chapter 5}

\section{Calibration of the Battery Model}

Following the development of the li-ion battery model in ESP-r [25] in the previous chapter, this chapter demonstrates how the model can be calibrated to represent a RES system. Section 5.1 reviews the data provided in this work to calibrate the default li-ion parameters, and section 5.2 reviews these parameters and how to determine them.

\subsection{Calibrated source data}

To invoke the use of the li-ion battery model, an electric network must be defined for a building model in ESP-r. Through ESP-r's graphical user interface, known as the Project Manager, the user can add components to the electric network. All the components must be connected to nodes as discussed in section 2.2. Nodes are connected via conductors previously created in ESP-r.

Upon adding the li-ion battery to the electric network, ESP-r will prompt the user to apply new parameter values or accept default ones. The default parameters have been assigned in this work, and have been calibrated to simulated data from a model created by NRC-ICPET [48]. The ICPET model is a one-dimensional discretization of a li-ion cell, into 100 partitions, using a finite difference approach as described in section 4.2. The model uses $\mathrm{LiC}_{6}$ and $\mathrm{LiMn}_{2} \mathrm{O}_{4}$ as the active material in the anode and the cathode, respectively. The simulations required the solution of four, highly coupled, partial differential equations driven by the flux of lithium ions. These equations are the electrolyte phase lithium ion concentration and electrical potential, solid phase potential solved in independent anode and cathode sub-domains, and the solid phase lithium ion concentrations, representing the interior of spherical particles 


\begin{tabular}{cc}
\hline Current density $A \cdot \mathrm{m}^{-2}$ at $25^{\circ} \mathrm{C}$ & Temperature ${ }^{\circ} \mathrm{C}$ at $7 \mathrm{~A} \cdot \mathrm{m}^{-2}$ \\
\hline \hline 0.35 & -40 \\
\hline 0.70 & -20 \\
\hline 1.40 & 0 \\
\hline 7.00 & 10 \\
\hline 35.0 & 20 \\
\hline 70.0 & 30 \\
\hline 140. & 40 \\
\hline OCV & 60 \\
\hline
\end{tabular}

Table 5.1: List of data sets provided by NRC-ICPET. 16 data sets of discharge curves were provided, each one at either constant current or constant temperature. [48]

distributed spatially in the anode and cathode regions of the battery. The interested reader is referred to the report by Darcovich et al. [48] for more information on this model's formulation.

The provided data was in sets of discharge curves of a single cell over several current densities, in $A \cdot m^{-2}$, and temperatures, in ${ }^{\circ} C$. The 16 data sets, each at a specific constant current density or constant temperature, are tabulated in table 5.1. Each of the constant current density data sets was simulated at a temperature of $25^{\circ} \mathrm{C}$. Each of the constant temperature data sets was simulated at a current density of $7 \mathrm{~A} \cdot \mathrm{m}^{-2}$. Current density is the amount of current discharged per surface area between the cathode and the anode, separated by the electrolyte. Manufactured cells vary in size from wrist watch "coin" shape cells, with the surface area of the coin itself, to high power "accordion" cells. These accordion cells are large sheets that have been folded into a compact rectangular pouch. The surface area for current density is the summation of the surface area of all the folds in the pouch. The number of these folds are limited by heat generation issues from concentrations of lithium ions.

It should be noted that the OCV curve was actually a simulation with a current density of $0.01 \mathrm{~A} \cdot \mathrm{m}^{-2}$. The nature of the simulations required that a current be declared, and it was assumed that an extremely low current such as $0.01 \mathrm{~A} \cdot \mathrm{m}^{-2}$ would have negligible losses and mimic the OCV. This assumption will be revisited 
later.

Each discharge set provided had the following columns of data: the time of the simulation in seconds, the cell voltage, the percentage of lithium content in the anode and the percentage of lithium content in the cathode. An example of these data sets is tabulated in Appendix A. Each simulation began with a typical lithium content of around $56 \%$ in the anode, chosen by Darcovich et al. [59], and ran until this depleted to $10 \%$. These simulation limits could be put into a context of DOD by assuming that a lithium content of $56 \%$ in the anode indicated a fully charged cell and a content of $0 \%$ indicated a fully empty one. Thus, the DOD of the cell at any time during the simulation could be expressed using the following:

$$
D O D=1-\frac{L i_{\text {anode }}^{+}}{\left(L i_{\text {anode }}^{+}\right)_{0}}
$$

where $L i_{\text {anode }}^{+}$is the percentage of lithium ion content in the anode at any time during

the simulation and $\left(L i_{\text {anode }}^{+}\right)_{0}$ is the initial percentage of lithium content in the anode (56\%). This meant that the simulations went from a DOD of 0 to around 0.82 . For more information on the simulated data from ICPET, the reader is referred to a report by Darcovich et al. [59].

The data was used to calibrate a default theoretical battery with a $6 \mathrm{~kW} \cdot \mathrm{h}$ capacity and a nominal voltage of $120 \mathrm{~V}$. These parameters come from an initial estimation of the capacity required for RES, based closely to the capacity required in electric vehicles. The battery in this work will be made up of accordion style cells, each having 18 folds, and measuring $8 \mathrm{~cm}$ by $12 \mathrm{~cm}$, or a total active material surface area of $0.1728 \mathrm{~m}^{2}$. These dimensions mimic a typical li-ion cell for a high power application. The following section describes how the data from ICPET [59] was used to calibrate the li-ion model.

\subsection{Calibration parameters}

The complete list of calibration parameters is shown in Appendix B. The values of these parameters can be altered through the Project Manager, or through editing the electric network file of the model. Section 5.2.1 describes the geometric parameters of the battery, and the set points to keep it within safe limits, and section 5.2.2 describes the parameters expressing the battery's performance with respect to current 
and temperature. It should be noted that the calibration in this work was with simulated data, and not a real cell (see section 5.1). Consequently, many of the parameters to describe the safe limits of the battery that would have been provided by a manufacturer are not available. Certain assumptions will be made throughout the following sections towards applying realistic values on these limits.

\subsubsection{Geometric parameters}

Parameters one and two in Appendix B are the number of cells in parallel and in series that make up the battery, respectively. Cells in series increase the overall battery voltage and cells in parallel increase the overall battery capacity, as described in section 4.1. If each cell has a typical nominal voltage found in the literature of $3.5 V$ [49], at least 35 cells are needed in series to achieve an overall nominal battery voltage of $120 \mathrm{~V}$. Dividing the desired overall battery capacity of $6 \mathrm{~kW} \cdot h$ by the nominal battery voltage of $120 \mathrm{~V}$ expresses the capacity in $A \cdot h$. Hence, for a desired overall battery capacity of $50 \mathrm{~A} \cdot h, 34$ cells will need to be placed in parallel, if each cell has a reference capacity of $1.5 A \cdot h$ (elaborated on further). If this were a real battery made up of a total of almost 1200 cells (34 by 35), special attention would have to be made by the BMS for cell balancing and identifying damaged cells.

Parameters three and four in Appendix B are the reference capacity and temperature for a single cell. The reference capacity is the energy gained from a cell in discharge with theoretically zero losses, in $A \cdot h$, at the reference temperature. The capacity assumes a constant nominal voltage, although in reality the voltage decreases over discharge. The reference temperature is the nominal temperature that the battery should operate at, in ${ }^{\circ} \mathrm{C}$. The values for both parameters should be available from the manufacturer's data for a commercial cell. The cell used in this work has a reference capacity of $1.5 \mathrm{~A} \cdot \mathrm{hr}$ and a reference temperature of $25^{\circ} \mathrm{C}$. The capacity was determined using the lower current density data sets from ICPET [59], where internal losses were minimal, and the following equation:

$$
C a p_{r e f}=\frac{S A \times I \times t_{\text {final }}}{D O D_{\text {final }}}
$$

where $S A$ is the surface area $\left(0.1728 \mathrm{~m}^{2}\right), I$ is the current density of the data set in $A \cdot m^{-2}, t_{\text {final }}$ is the total simulation time of the data set and $D O D_{\text {final }}$ is the final DOD of the data set. 
Parameters five and six in Appendix B are the upper and lower allowable voltages for a cell, respectively. Using a polynomial fit to the OCV (elaborated further), this corresponded to an upper cell voltage of $4.20 \mathrm{~V}$ and a lower cell voltage $3.15 \mathrm{~V}$ for a DOD of 0 and a DOD of 1 , respectively.

Parameters seven and eight in Appendix B are the maximum allowable current and voltage of a single cell. Manufacturers often limit the current to a maximum value before irreparable damage occurs due to overheating, and the voltage to a maximum value before its SOC becomes dangerous at the electrochemical level. For this work, the maximum cell current in charge or discharge allowed is $24.2 \mathrm{~A}$, and the maximum cell voltage allowed is $4.3 \mathrm{~V}$. The current is taken from the maximum current given in the data from ICPET [59], and the voltage is just beyond a corresponding DOD of 1 . For 34 cells in parallel, this would result in an overall maximum current of over $800 A$ and for a real battery may cause safety concerns. It is re-iterated that this calibration is for a theoretical battery and that manufacturers would have values based on safety through experimentation.

Parameters nine and ten in Appendix B are the initial temperature and DOD of the battery. These are the values that the simulation is initialized with. Typically, the initial temperature would be the reference temperature of the battery, in our work $25^{\circ} \mathrm{C}$. This work, by default, will also assume that the battery begins fully charged, so the initial DOD is 0 .

Parameter 11 in Appendix B is the thermal mass of a single cell. The thermal mass is the mass, $m$, of a single cell multiplied by its specific heat, $C_{p}$. While in reality, this value would vary over temperature and voltage because the $C_{p}$ varies with these properties, the model assumes that this is constant. Hassoun [16] reports that typical energy densities for li-ion cells range from $150 \mathrm{Wh} \cdot \mathrm{kg}^{-1}$ to $250 \mathrm{Wh} \cdot \mathrm{kg}^{-1}$ at a nominal voltage of $3.5 \mathrm{~V}$. Considering that the cells in this work have a reference capacity of $1.5 \mathrm{~A} \cdot h$, or $5.25 \mathrm{~W} \cdot h$ when multiplied by their nominal voltage, the mass of a single cell can be determined by dividing this capacity by the energy density. Taking an average energy density of $200 \mathrm{Wh} \cdot \mathrm{kg}^{-1}$, the mass of a single cell would be $26.25 \mathrm{~g}$. Maleki [60] reports a $C_{p}$ of $1.04 \pm 0.02 \mathrm{~J} \cdot \mathrm{g}^{-1} \cdot \mathrm{K}^{-1}$ at an OCV of $3.75 \mathrm{~V}$. For the calibration of an actual cell, the manufacturer would report the mass. The $C_{p}$ would ideally be given as well, however, the value reported here would provide a reasonable assumption. Using the reported energy density from Hassoun [16] and the reported $C_{p}$ from Maleki [60], the thermal mass of the cell in this work is: 


$$
m \cdot C_{p}=27.3 J \cdot{ }^{\circ} C^{-1}
$$

Parameter 12 in Appendix $\mathrm{B}$ is the heat loss factor for the battery, $h A$, as described in section 4.3. $A$ is the surface area of the battery, in $m^{2}$, which in this case is made up of 34 cells in parallel and 35 in series. Each cell is $8 \mathrm{~cm}$ by $12 \mathrm{~cm}$, with a thickness of $2 \mathrm{~cm}$ for 18 folds, corresponding to an overall battery dimension of $272 \mathrm{~cm}$ by 12 $\mathrm{cm}$ by $70 \mathrm{~cm}$. This corresponds to an outer battery surface area, $A$, of $4.63 \mathrm{~m}^{2}$ (see fig. 5.1).

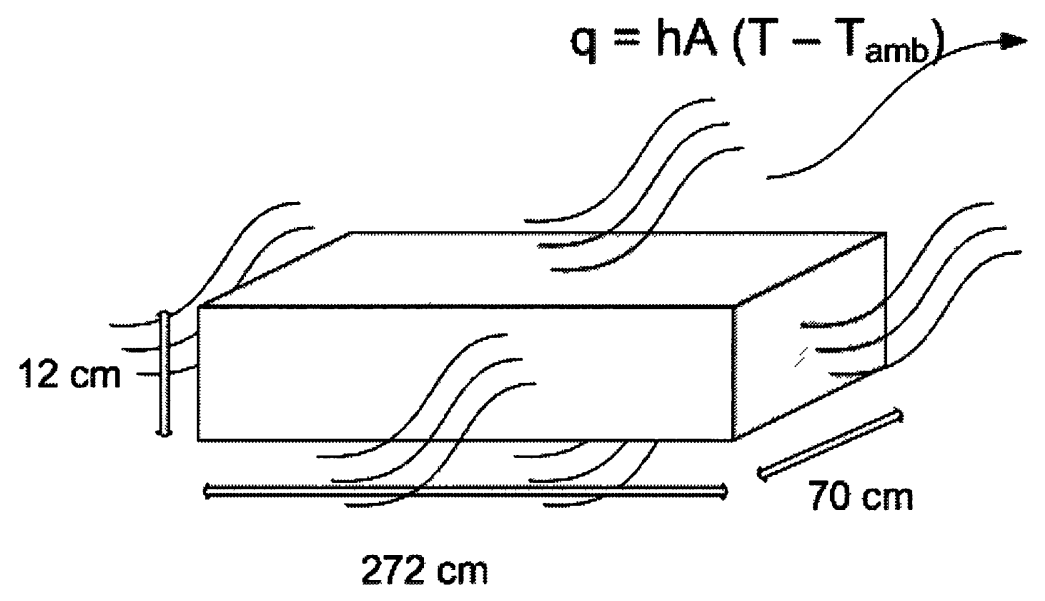

Figure 5.1: Schematic of heat loss to surroundings from battery pack.

The heat transfer coefficient between the battery and its surroundings, $h$, is in $W \cdot m^{-2} \cdot{ }^{\circ} C^{-1}$. This linear coefficient can encompass all, or any forms of heat transfer, i.e., conductive, convective and radiative, and is assumed constant over all temperatures. In this work, it is assumed that only convection and radiation contribute to heat transfer. Assuming that convection is treated as laminar and over all the surfaces of the battery, Holman [56] uses the following empirical equation for free convection of air at atmospheric pressure over a horizontal plane:

$$
h_{c}=1.32\left(\frac{\Delta T}{L}\right)^{1 / 4}
$$

where $h_{c}$ is the convective heat transfer coefficient, in $W \cdot m^{-2} \cdot{ }^{\circ} C^{-1}, \Delta T$ is the difference in temperature between the surface and the ambient, and $L$ is a vertical 
dimension in $m$. Thus, using a gradient of $35{ }^{\circ} \mathrm{C}$, the gradient for the maximum allowable battery temperature and an ambient temperature of $25^{\circ} \mathrm{C}$, and an $\mathrm{L}$ value of $2.72 \mathrm{~m}$ for the width of the battery, the convective heat transfer coefficient is 2.50 $W \cdot m^{-2} \cdot{ }^{\circ} C^{-1}$.

The longwave radiative exchange between the battery and its environment is expressed by the following:

$$
q_{r a d}=A \sigma \epsilon\left(T_{b}^{4}-T_{a}^{4}\right)
$$

where $q_{r a d}$ is the radiative exchange in $W, A$ is the same surface area reported above, $\sigma$ is the Stefan-Boltzmann constant, equal to $5.669 \times 10^{-8} \mathrm{~W} \cdot \mathrm{m}^{-2} \cdot K^{-4}[56], \epsilon$ is the emissivity of the battery relative to that of a blackbody, and $T_{b}$ and $T_{a}$ are the battery and room temperatures, in $K$, respectively.

Assuming that the battery enclosure is electrically insulated with a material such as rubber, an $\epsilon$ value of 0.94 taken from Holman [56] is used. The entire equation is made linear using an equivalent radiative coefficient, $h_{r}$ in $W \cdot m^{-2} \cdot{ }^{\circ} C^{-1}$ :

$$
A h_{r}\left(T_{b}-T_{a}\right)=A \sigma \epsilon\left(T_{b}^{4}-T_{a}^{4}\right)
$$

By re-arranging this equation, and using a battery temperature of $60^{\circ} \mathrm{C}(333 \mathrm{~K})$ and a zone temperature of $25^{\circ} \mathrm{C}(298 \mathrm{~K}), h_{r}$ is $6.71 \mathrm{~W} \cdot \mathrm{m}^{-2} \cdot{ }^{\circ} \mathrm{C}^{-1}$.

When the convective and radiative terms are summed together, and multiplied by the surface area above, the heat loss factor in this work is:

$$
h \cdot A=42.64 W \cdot{ }^{\circ} C^{-1}
$$

Many assumptions have been made to describe the heat transfer process of the battery for this work. More analysis into the sensitivity of these parameters may be necessary. This will be elaborated in the future work section in chapter 7 .

Parameter 13 in Appendix B is the built in resistance heater of each cell in $\Omega$. This resistance heater is used by the battery if its temperature drops below an acceptable value, as discussed in section 4.4. Because the battery is made up of several cells in series and parallel, this value must be selected per cell to give a realistic overall heater resistance (see fig 5.2).

The overall resistance of a string of resistors in series is their sum, and the inverse 


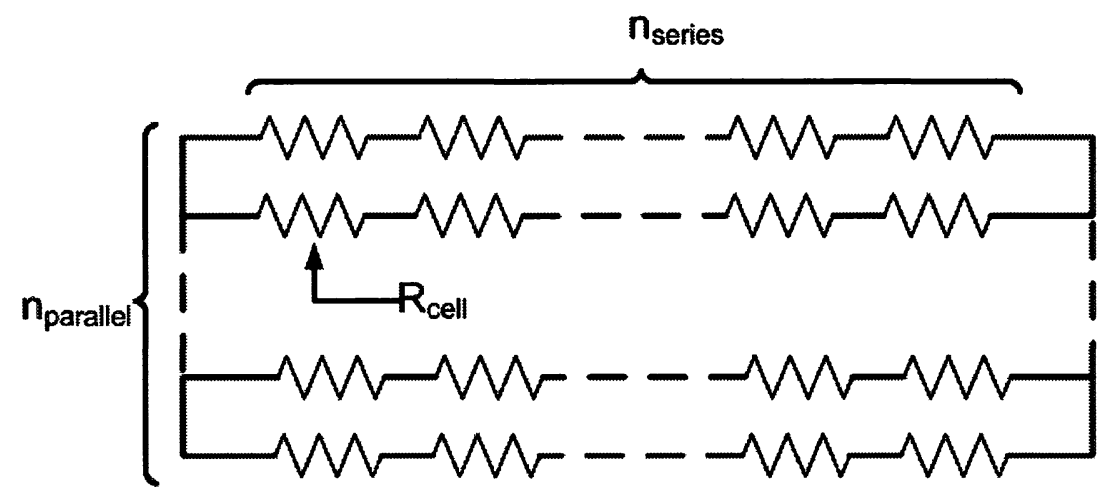

Figure 5.2: Schematic of cell resistors in series and parallel making up an overall battery resistance.

of the overall resistance of a string of resistors in parallel is the summation of the inverse of each string. Thus, the overall resistance is given by the following equation:

$$
R_{\text {battery }}=\frac{n_{\text {series }}}{n_{\text {parallel }}} R_{\text {cell }}
$$

In this work, an overall resistance of $600 \Omega$ is sought. This would equate to a battery heating of $24 \mathrm{~W}$ at a nominal voltage of $120 \mathrm{~V}$ (see eq. 4.17). Thus, each cell must have a resistance of $582.86 \Omega$. This value of $24 \mathrm{~W}$ may need to change with further testing.

Parameters 14 and 15 in Appendix B are the upper and lower DOD limits of the battery. These are used in the BMS to ensure that the battery stays within safe limits and should correspond to the upper and lower voltages given in parameters five and six. Typical battery DOD limits for a commercial cell would be between 0 and 0.8 . If an empty battery DOD limit of 0.8 was chosen for this work, the final cell voltage would be $3.52 \mathrm{~V}$. However, because a nominal cell voltage of $3.5 \mathrm{~V}$ is desired for this work, a DOD range from 0 to 1 is chosen. This places the lower cutoff voltage at $3.15 \mathrm{~V}$ and the nominal voltage close to the desired.

Parameters 16 and 17 in Appendix B are the upper and lower temperature limits of the battery. These are used in the BMS to ensure that the battery stays within safe limits. For this work, the upper and lower limits are $60^{\circ} \mathrm{C}$ and $-40^{\circ} \mathrm{C}$, respectively. These limits should be available from the manufacturer for a commercially available cell.

Parameters 70 and 71 in Appendix B describe the self-discharge parameters for a 
single cell of the battery, described in section 4.1. Parameter 70 is the self-discharge constant of a cell, $k_{o}$ in Ampere, and parameter 71 is the self-discharge activation energy of a cell, $E_{a s}$ in $k J \cdot \mathrm{kmol}^{-1}$ (see eq. 4.4). These values are difficult to determine and may have to be done through experimentation. Although self-discharge has been reported to be typically very low (see section 1.2.3), this functionality was included in the model. In this work, and by default, these values will be zero and hence self-discharge will be ignored. Any simulations with extended periods of battery idling, however, may require an accurate treatment of these parameters through experimentation.

Parameter 72 in Appendix B is the zone in the house model where the li-ion battery is contained. This is the zone that the battery does its thermal exchange with. By default this value will be 1 to indicate that the battery is in the first zone of the ESP-r model.

Parameter 73 in Appendix B is the parasitic power of the entire battery in Watts. This is the power required from the BMS circuitry to run its functions. Published values for the consumption of a typical BMS are hard to come by, because it is a relatively new technology. In this work, the parasitic power will be assigned a value of zero, however, future work might determine a more realistic value.

Parameter 81 in Appendix B is the number of cycles indicating a full battery life. As described in section 4.3, the model has been implemented with degradation. At the end of every time-step, the absolute value of the change in DOD is recorded. These are summed up over the simulation to determine the number of cycles that the battery has used towards its lifetime. The battery's capacity will linearly decrease to $80 \%$ of its original capacity at the cycle value given in parameter 81 . After this, the battery's capacity will diminish to zero over the next $10 \%$ of the cycle amount given in parameter 81. From the literature, a value of 3000 cycles [18] will be used as the default for parameter 81 . It should be noted that this value is optimistic compared to other values found [19].

Now that the geometric parameters have been defined, it is necessary to describe the relationship of the battery's performance with current and temperature. The following section explains this procedure. 


\subsubsection{Current and temperature parameters}

Parameters 18 to 23 in Appendix B are the five coefficients used to describe the OCV of a cell as a function of DOD using a 5th order polynomial fit as shown in equation 5.9 below.

$$
O C V(D O D)=E_{5} \cdot D O D^{5}+E_{4} \cdot D O D^{4}+E_{3} \cdot D O D^{3}+E_{2} \cdot D O D^{2}+E_{1} \cdot D O D+E_{0}
$$

Figure 5.3 shows the plot of a 5th order polynomial fit to the OCV data provided from ICPET [59]. This was done using a method of least squares. The figure also shows the coefficients used in the 5th order polynomial. A commercially available cell would have a similar plot to determine the OCV coefficients.

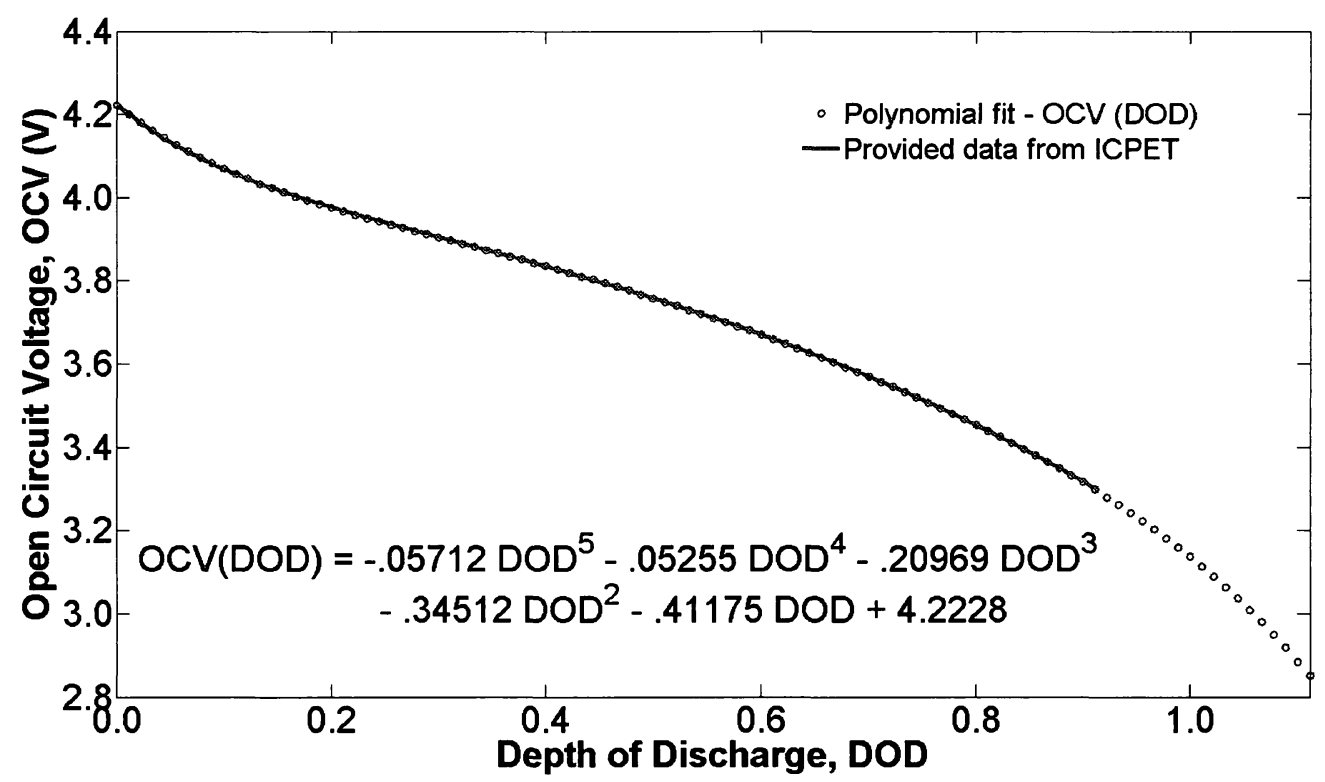

Figure 5.3: OCV polynomial fit using a least squares method to original simulated data from ICPET [59].

This polynomial equation was used in the work above to determine certain parameters, e.g., the cutoff voltage at a DOD of 1 , because the original simulations from ICPET [59] were not run to this point. It is this reference OCV curve that will be translated and transformed to describe the performance of the battery over a range 
of temperatures and currents.

It should be noted again that this was in actuality not the true OCV curve. The nature of the simulations from ICPET [59] required that a current be given; so a low current density of $0.01 \mathrm{~A} \cdot \mathrm{m}^{-2}$ was used, corresponding to a current of $0.0017 \mathrm{~A}$ for the theoretical cell used in this work.

To justify this assumption, a plot of the polynomial fits to all of the constant current data sets from ICPET [59] (see table 5.1) is shown in figure 5.4. In this plot, the low current curves seem to have negligible voltage drops and capacity reductions, indicating that the internal losses at these currents are insignificant. The $0.0017 \mathrm{~A}$ curve seems to be acceptable in representing the OCV of this cell.

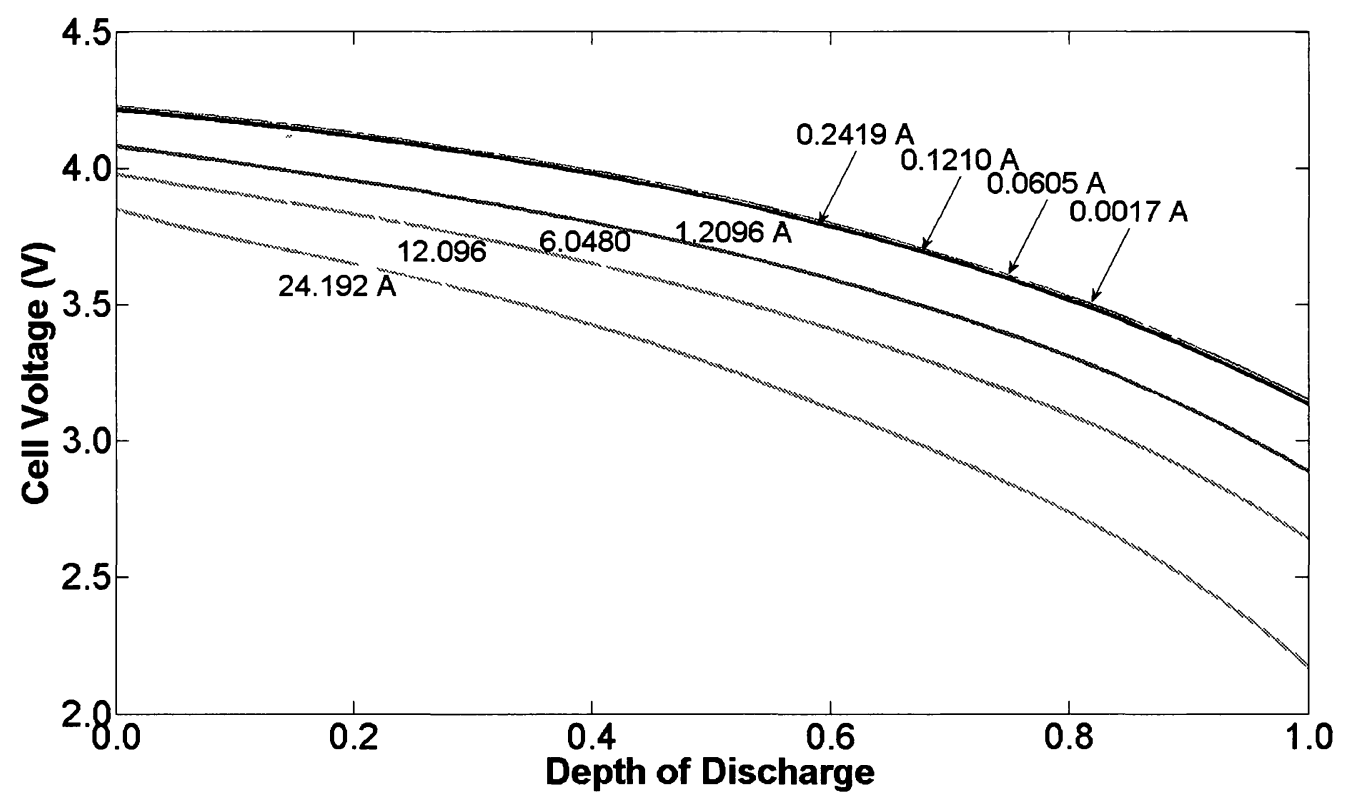

Figure 5.4: Voltage as a function of DOD over a range of currents (data sourced from ICPET [59]).

Parameters 24 to 29 in Appendix B are the five coefficients used to describe the cell's internal resistance as a function of DOD using a 5 th order polynomial fit as shown in equation 5.10 below. 


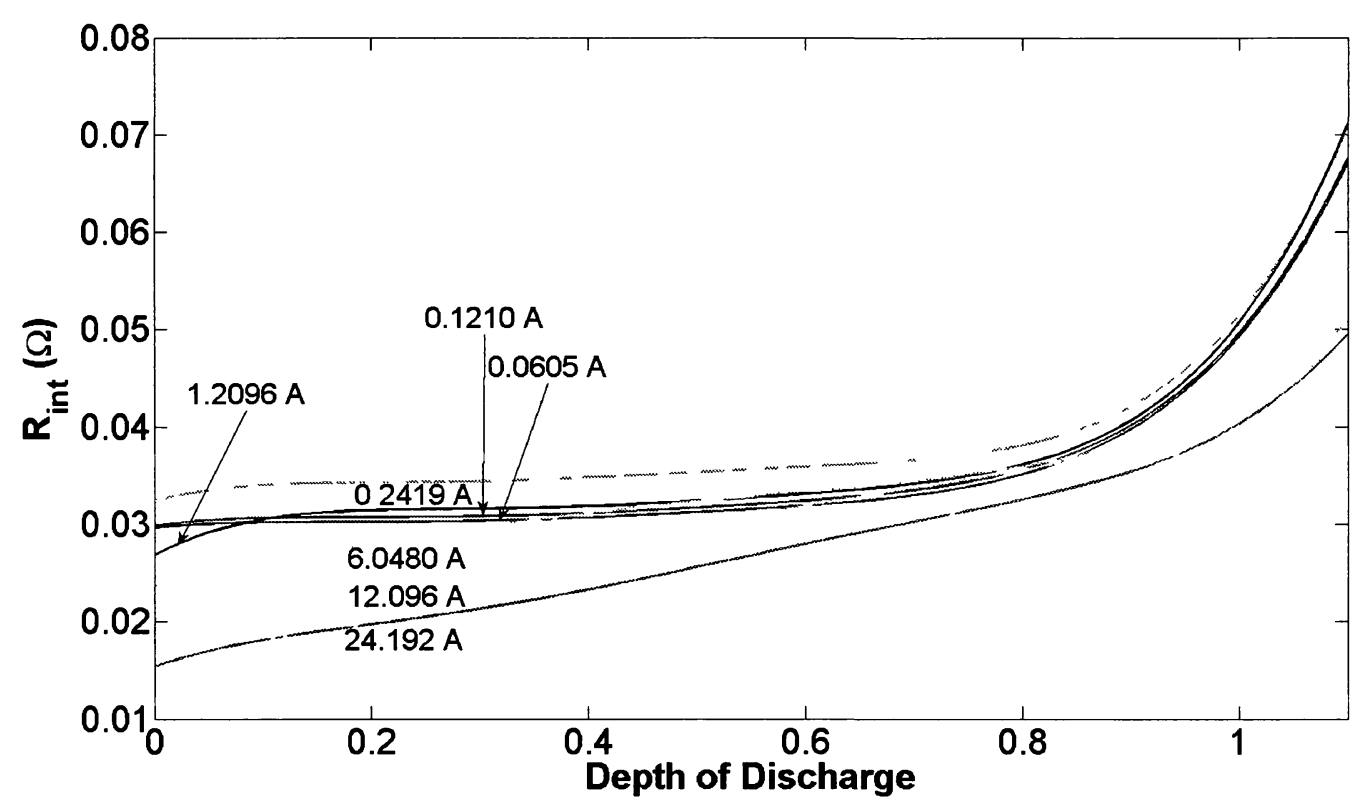

Figure 5.5: Internal resistance as a function of DOD over a range of currents (data sourced from ICPET [59]).

$R_{\imath n t}(D O D)=R_{\imath n t 5} \cdot D O D^{5}+R_{\imath n t 4} \cdot D O D^{4}+R_{\imath n t 3} \cdot D O D^{3}+R_{\imath n t 2} \cdot D O D^{2}+R_{\imath n t 1} \cdot D O D+R_{\imath n t 0}$

The original approach used for this modelling work assumed that the internal resistance was only a function of DOD and was linear over a range of currents (see section 4.3). To verify this assumption using the data provided by ICPET [59], the internal resistance as a function of DOD for each constant current data set is determined. This is done by taking the difference between the voltage curve of the OCV and the voltage curve at a specific constant current, and divided by the current itself, i.e., :

$$
R_{\imath n t}(D O D, I)=\frac{O C V(D O D)-V_{I}(D O D, I)}{I}
$$

If the internal resistance is only a function of DOD, and thus linear over varying currents, a plot of the internal resistance curves should lay upon each other. Figure 5.5 shows this plot using the data provided. 

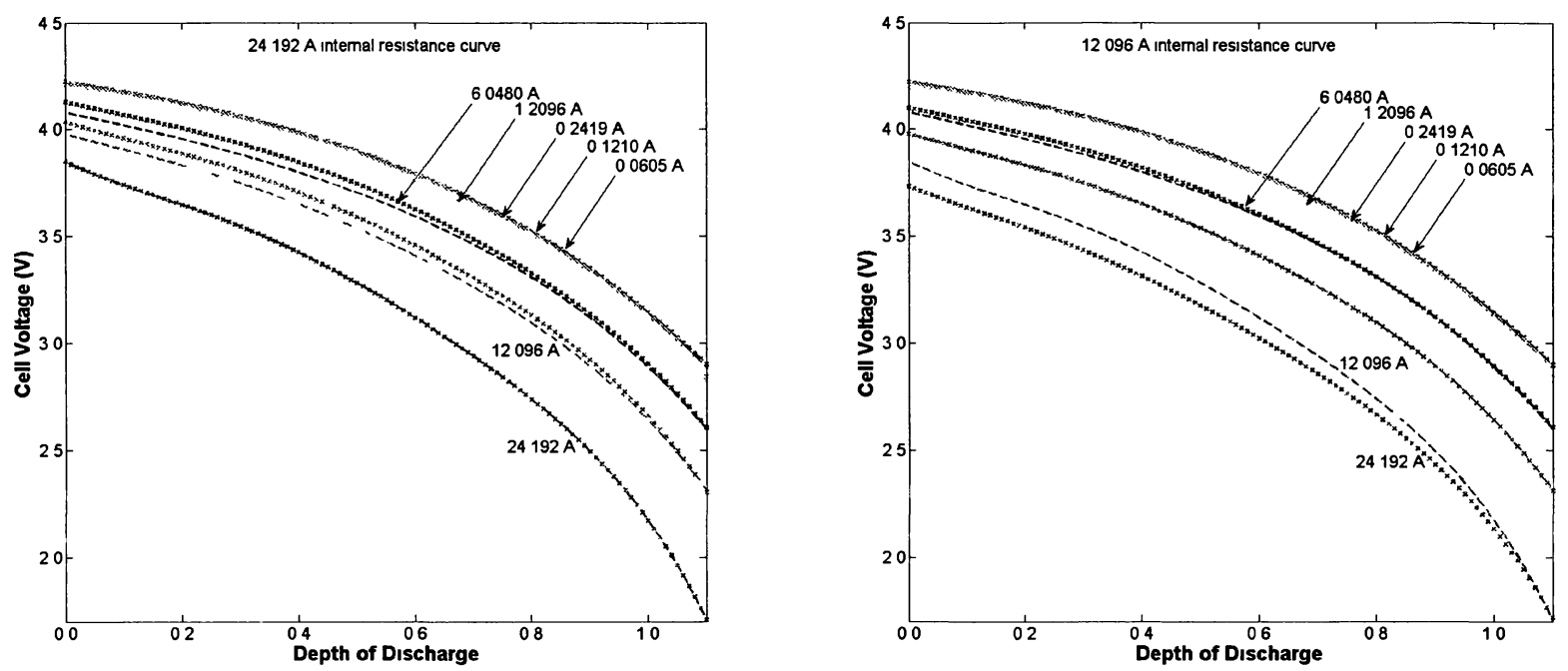

Figure 5.6: Plot of polynomial fit of original data from ICPET [59], shown as dashed lines, compared with an OCV curve scaled by an internal resistance curve, shown as " $\mathrm{x}$ " $\mathrm{s}$.

From this plot, it seems that the assumption holds quite well, specifically at lower currents. As the current increases however, the internal resistance does not remain constant, indicating that the linear assumption does not hold. It is necessary to choose an internal resistance curve to represent the internal resistance of the cell over a range of currents.

Two plots are compared in figure 5.6. The plot on the left shows the polynomial fits to the original data from ICPET [59] compared with a scaled OCV curve using the $24.192 A$ internal resistance curve. The plot on the right shows the polynomial fits to the original data from ICPET [59] compared with a scaled OCV curve using the $12.096 \mathrm{~A}$ internal resistance curve. Both fits seem to represent the low current values reasonably. This is because regardless of the subtle changes in internal resistance, when multiplied by small currents they become negligible. The plot on the left obviously fits the $24.192 \mathrm{~A}$ data well, but not so much the other high currents. The plot on the right fits the high currents quite well, obviously the $12.096 \mathrm{~A}$ the best, however, it does not fit the $24.192 A$ curve well. This implies that internal resistance is a function of DOD and current, varying due to changes at the electrochemical level, and that the modelling assumptions made in section 4.3 are not valid.

To compensate for the change in internal resistance at high currents, an additional 


\begin{tabular}{ccc}
\hline Symbol & Value if below 16 A & Value if above or equal to 16 A \\
\hline \hline$R_{5}$ & 0.1152 & 0.1972 \\
\hline$R_{4}$ & -0.2556 & -0.4749 \\
\hline$R_{3}$ & 0.2007 & 0.4118 \\
\hline$R_{2}$ & -0.0651 & -0.1472 \\
\hline$R_{1}$ & 0.0265 & 0.0380 \\
\hline$R_{0}$ & 0.0202 & 0.0154 \\
\hline
\end{tabular}

Table 5.2: Internal resistance coefficients used for the model calibration.

set of internal resistance parameters have been included in the model. They are listed as parameters 74 to 79 in Appendix B, and are a second set of internal resistance coefficients to be used in the case of high discrepancies in internal resistance between discharge curves. Parameter 80 is a current value. If invoked, the model will use the original internal resistance coefficients (parameters 24-29) for battery currents less than the current specified in parameter 80 , and use the second set of coefficients (parameters 74-79) for higher battery currents. If the user chooses to only use one set of coefficients, they need to enter a value of (999.) as any value for parameters 74 to 80 , and the model will not make use of them. In which case only the original set will be used.

Table 5.2 shows the coefficients used in this work. The current limit chosen, i.e., parameter 80 , is $16 \mathrm{~A}$. In other words, if the current imposed is less than $16 \mathrm{~A}$, the model will use the first column of coefficients to calculate internal resistance, entered as parameters 24-29; otherwise, it will use the second column for higher currents (parameters 74-79).

Parameters 30 to 45 in Appendix B are used to adjust the reference capacity of the battery to account for changing current, using the $\alpha$ factors discussed in section 4.3 (see eq. 4.8). The model was developed to accept eight different discharge/charge curves at eight different currents. For each current specified, there should be an accompanying $\alpha$ factor. In other words, the lowest discharge current will be entered as parameter 30 and its $\alpha$ factor will be 31 , then the next current will be entered as parameter 32 and its $\alpha$ factor will be 33, etc. 


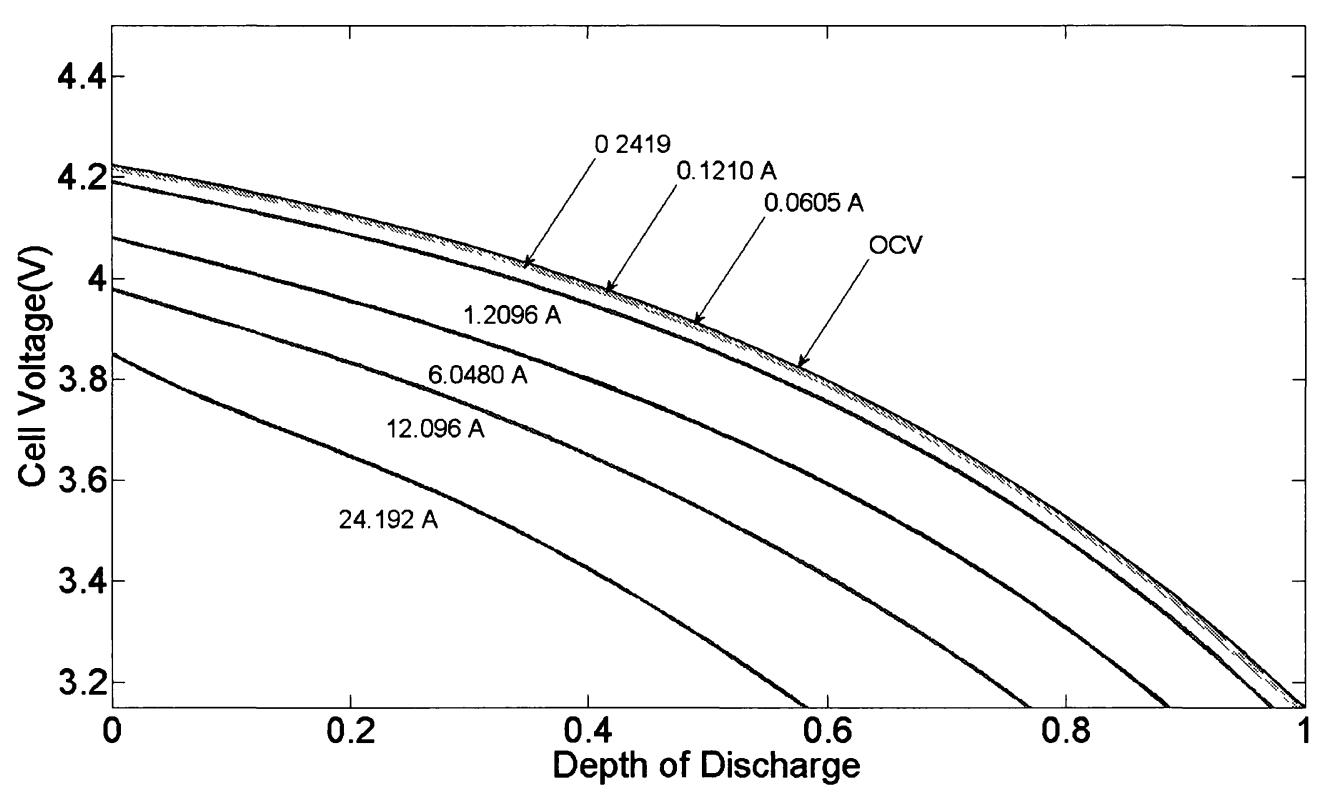

Figure 5.7: Voltage as a function of DOD over a range of currents.

To obtain these values, a plot must be made of the cell voltage as a function of DOD for all the curves similar to figure 5.7. This plot should be limited in the y-axis range to the lower cutoff voltage, in this work, $3.15 \mathrm{~V}$. The $\alpha$ factors can be found by measuring the difference in cell capacity relative to the OCV curve as discussed in section 4.3 (see fig. 4.6).

Table 5.3 shows the $\alpha$ factors used in the model calibration for this work. These factors will be used and the model will linearly interpolate between them to size the cell capacity for a varying imposed current. It should be noted that there were only seven discharge sets provided by ICPET [59], although the model requests eight. In this case, the user needs to input a value of (999.) as the parameter to describe the unavailable current, and keep the same $\alpha$ value used for the the previously given current, to override this last curve. A different user wishing to calibrate a new cell, can then have as few discharge sets of data, at the sake of losing accuracy, or as many as eight.

Similar transformations performed to the OCV curve are done to account for temperature. These temperature variations are applied to the model using parameters 


\begin{tabular}{cc}
\hline Current (A) & $\alpha$ \\
\hline \hline 0.0605 & 1.00 \\
\hline 0.1210 & 1.00 \\
\hline 0.2419 & 1.00 \\
\hline 1.2096 & 0.985 \\
\hline 6.0480 & 0.895 \\
\hline 12.096 & 0.775 \\
\hline 24.192 & 0.593 \\
\hline
\end{tabular}

Table 5.3: Internal resistance coefficients used for model calibration.

46 to 69 in Appendix B. The model allots for eight different temperature curves, and as discussed in section 4.3 , requires that for each temperature, in ${ }^{\circ} C$, a $\beta$ factor (see eq. 4.10) and a $\Delta V(T)$ drop in voltage value (see eq. 4.9) be declared. In other words, the lowest temperature will be entered as parameter 46 , its $\beta$ factor will be 47 and its $\Delta V(T)$ will be 48 . The next temperature will be entered as parameter 49 , its $\beta$ factor will be 50 and its $\Delta V(T)$ will be 51 , etc.

The original discharge curves for varying temperature data provided from ICPET [59] were performed at a current density of $7 A \cdot \mathrm{m}^{-2}$. This was because the simulation time to run at $0.01 A \cdot \mathrm{m}^{-2}$ would have been too long. Thus, to shift the provided data to simulate OCV curves at various temperatures, the internal resistance effects due to current had to be removed:

$$
O C V(T, D O D)=V(T, D O D)+I \cdot R_{\text {int }}
$$

where $O C V(T, D O D)$ is the OCV curve at a given temperature $T, V(T, D O D)$ is the original cell voltage provided, and $I \cdot R_{\text {int }}$ is the internal resistance due to current multiplied by the current itself, or in this case $1.2096 \mathrm{~A}\left(7 \mathrm{~A} \cdot \mathrm{m}^{-2}\right)$.

Assuming that these curves represent the true OCV curves at these temperatures, it is now possible to measure the difference in voltage, as temperature changes, with respect to the $25^{\circ} \mathrm{C}$ reference curve. This plot is shown below for each temperature curve as figure 5.8 .

From this figure, each voltage curve seems to have a reasonable constant voltage 


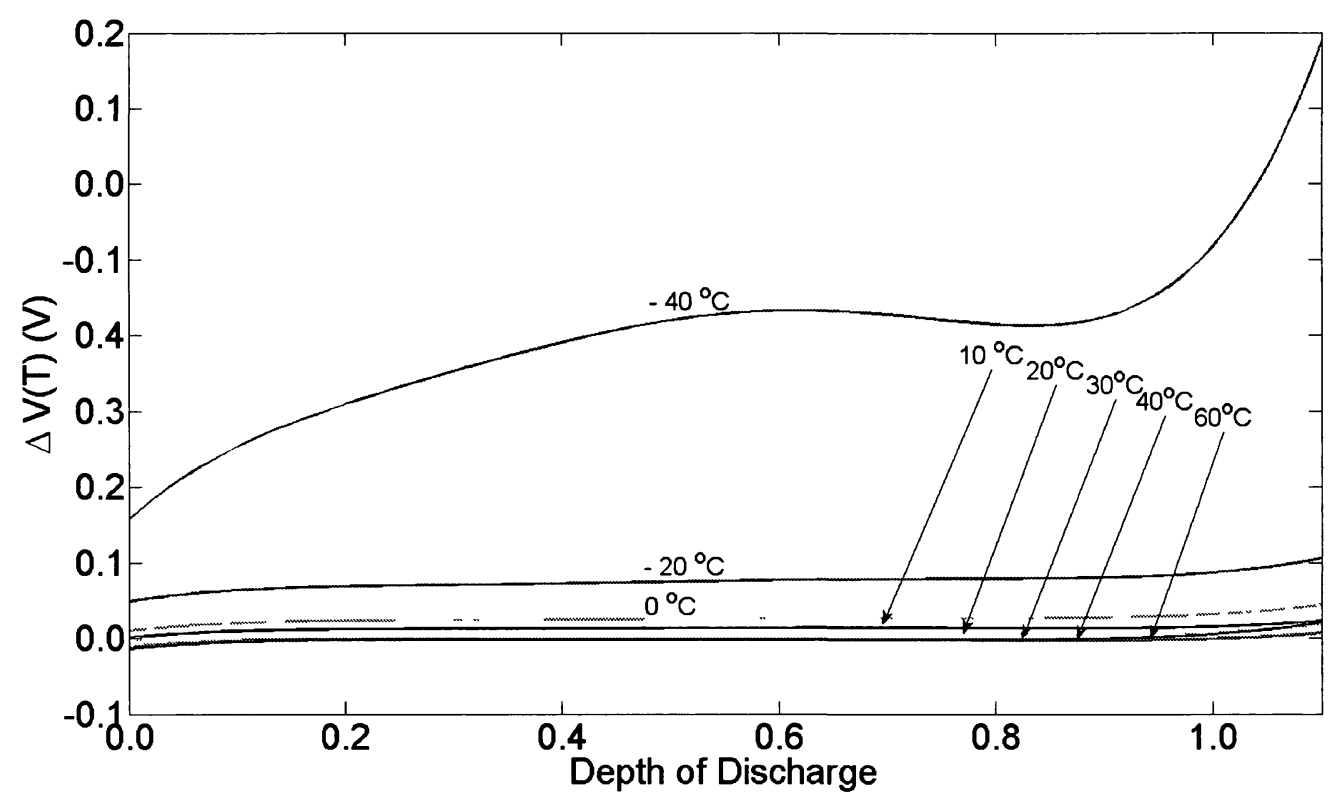

Figure 5.8: Change in voltage as a function of DOD due to cell temperature with respect to the $25^{\circ} \mathrm{C}$ reference curve.

difference, with the exception of the $-40^{\circ} \mathrm{C}$ case. In all the other cases, the change in temperature does not seem to affect voltage with respect to DOD. At extremely low temperatures however, the cell voltage is adversely affected with respect to DOD due to changes in the electrochemistry of the cell. Consequently, the average voltage drop over the entire DOD was taken, and it is assumed that this applied to the $-40^{\circ} \mathrm{C}$ case. Although this may not be accurate, it is assumed sufficient to model actual cell behaviour in rare occurrences when the battery would be at those temperatures.

The $\beta$ factors for temperature are found in the same way as the $\alpha$ factors, relating to current above. Figure 5.9 shows the temperature curves limited to the cutoff voltage of $3.15 V$, in order to determine the $\beta$ factors. Table 5.4 shows the average voltage drop, $\Delta V(T)$, and the $\beta$ factor, for each temperature. The model will linearly interpolate between these values to determine a suitable $\Delta V(T)$ and $\beta$, for a given cell temperature.

This chapter discussed all the parameters necessary to calibrate the li-ion model developed in this work. Its default calibration was performed with simulated data 


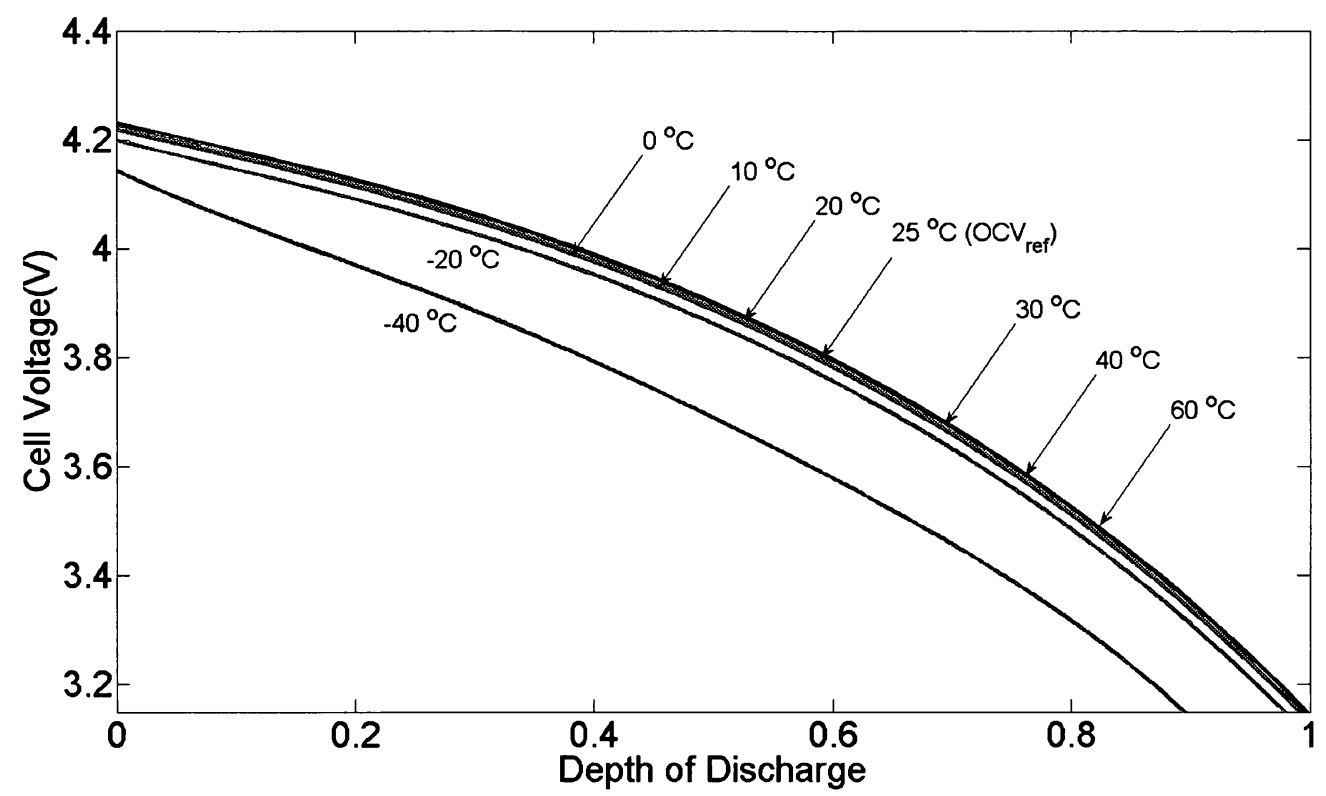

Figure 5.9: Change in voltage as a function of DOD due to cell temperature with respect to the $25^{\circ} \mathrm{C}$ reference curve.

from NRC-ICPET [48]. Using this calibrated model, and the data collected of nonHVAC and space cooling electrical loads, a micro-cogeneration device coupled with a li-ion model is now able to be assessed accurately using whole building simulation. The following chapter reviews the next step in achieving this. 


\begin{tabular}{ccc}
\hline Temperature $\left({ }^{\circ} \mathrm{C}\right)$ & $\Delta V(T)(V)$ & $\beta$ \\
\hline \hline-40 & 0.1870 & 0.905 \\
\hline-20 & 0.0366 & 0.985 \\
\hline 0 & 0.0123 & 1.00 \\
\hline 10 & 0.0062 & 1.00 \\
\hline 20 & 0.0024 & 1.00 \\
\hline 30 & $-1.4449 \times 10^{-5}$ & 1.00 \\
\hline 40 & -0.0012 & 1.00 \\
\hline 60 & -0.0012 & 1.00 \\
\hline
\end{tabular}

Table 5.4: $\Delta V(T)$ values to calibrate li-ion battery model. 


\section{Chapter 6}

\section{Demonstration of new modelling capabilities}

Chapter 3 reviewed the collection and analysis of Canadian residential non-HVAC and space cooling electric data. Chapters 4 and 5 reviewed the development and calibration a li-ion battery model in ESP-r. With the completion of these research objectives, this chapter demonstrates the new modelling capabilities developed in this work.

\subsection{Li-ion battery model debugging}

Inaccuracies in a model can take on many forms, preventing it from exhibiting the true characteristics of its physical counterpart. These errors can range from misinterpretation or simplification of the physical phenomena in the modelling approach, to errors in the code used to represent the model. Regarding the former of these errors, they have been continuously addressed throughout sections 4 and 5. Many simplifications and assumptions have been made throughout the development of the li-ion model, such as the lumped capacitance approach and the treatment of the heat transfer process. The validity of these assumptions have been individually addressed in an effort to simplify the complexity of the physics of a battery, while obtaining a model with the necessary resolution for use in building simulation.

Finding syntax errors in the source code is a lengthy and ongoing process known as debugging. Using a pragmatic approach, the six basic battery operating modes were verified for functionality. These operating modes were normal battery charge and discharge, over battery charge and discharge, and over battery temperature and 
under battery temperature.

An example of a debugging error that was found is shown in figure 6.1. This error involved a calibration parameter being incorrectly read by the battery subroutine in the simulation. The specific parameter was a $\beta$ temperature factor as explained in section 43 . As a result, the battery discharged at an unexpectedly high rate as its temperature neared the temperature corresponding to the incorrect $\beta$ value. Upon discovery and remedy of this error, the battery discharged at the expected rate.

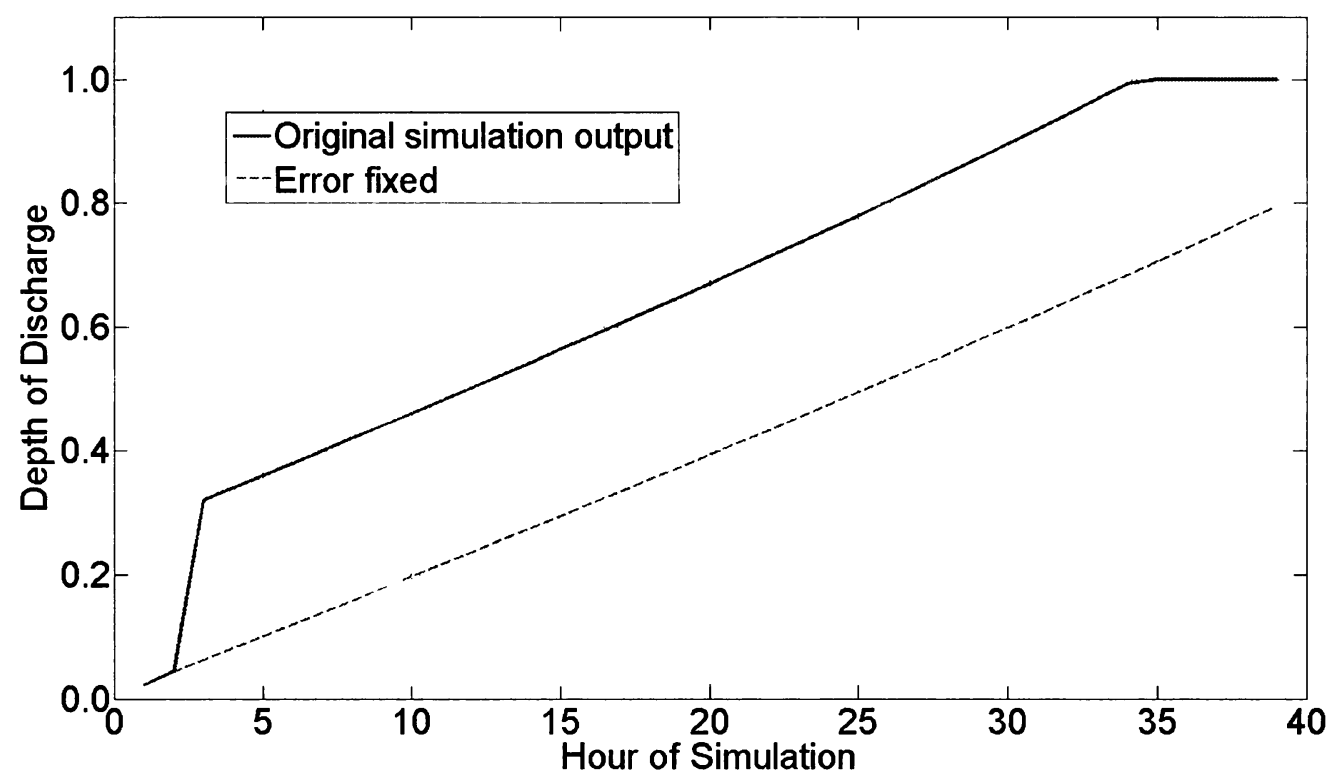

Figure 6.1: Example of coding error discovered during debugging.

Most, if not all of the errors have been accounted for, especially with regards to the basic operation modes discussed above. With these errors remedied, it is now possible to validate the model with external data.

\subsection{Validation with simulated data from ICPET}

The data from ICPET [59] used to calibrate the model was simulated at constant current. This way, the battery model developed in this work could account for changes in battery capacity and voltage at specific currents, and use linear interpolation between them to account for changes at any current. The electric loads imposed on the 
battery in ESP-r, however, are specified as a constant power draw over the time-step. To validate the model developed in this work, a new simulation was requested of ICPET for a cell discharging at constant power. The new simulation was a cell at $25^{\circ} \mathrm{C}$, discharging at a rate of $4.3 \mathrm{~W}$. The li-ion model developed in this work was modified to be a single cell with the same fixed temperature and the same imposed electric load. The results are shown in figure 6.2

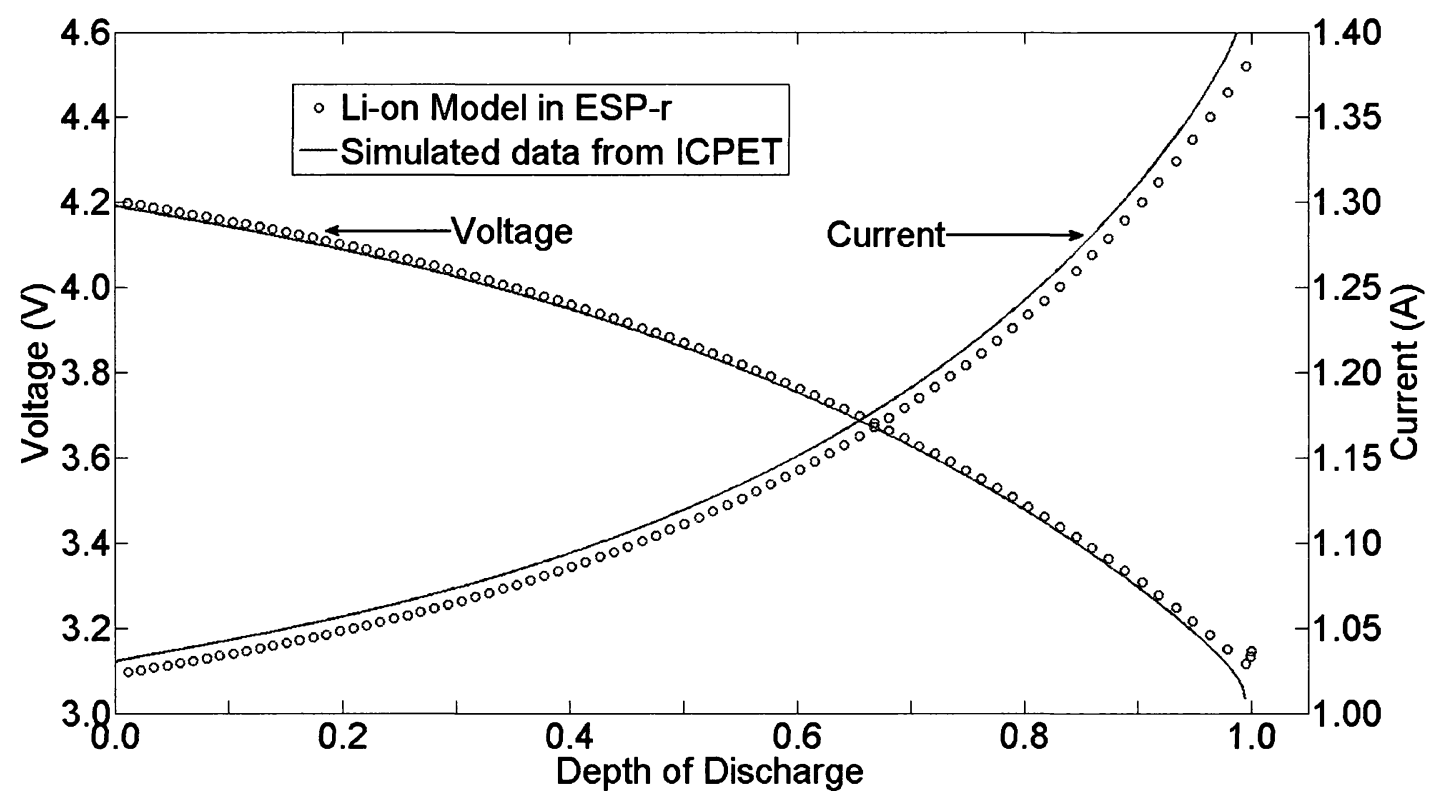

Figure 6.2: Comparison of li-ion simulation run in ESP-r with simulated data from ICPET [48].

It appears from this figure that the current calculated by ESP-r is lower than the simulated data from ICPET [48]. This discrepancy could be due to the sensitivity of the time-step chosen in ESP-r. In ESP-r, the current that the battery charges or discharges at cannot change within a time-step. However, because the electric load imposed on the battery over a time-step in ESP-r is constant, the current should vary because the voltage should vary. Because the voltage does not change within a time-step either, the subroutine in ESP-r uses an average voltage over the time-step based on the initial DOD and the predicted final DOD. The current calculated for the time-step is based on this average voltage.

This indicates that the longer the chosen time-step in a simulation is, the lower the 
average voltage is in a discharge scenario and vice versa in a charge scenario. Thus, for a longer time-step, the current will be lower in discharge and higher in charge. This is illustrated in figure 6.3. In this figure, the same simulation was performed with a discharging cell, but with increasing time-steps.

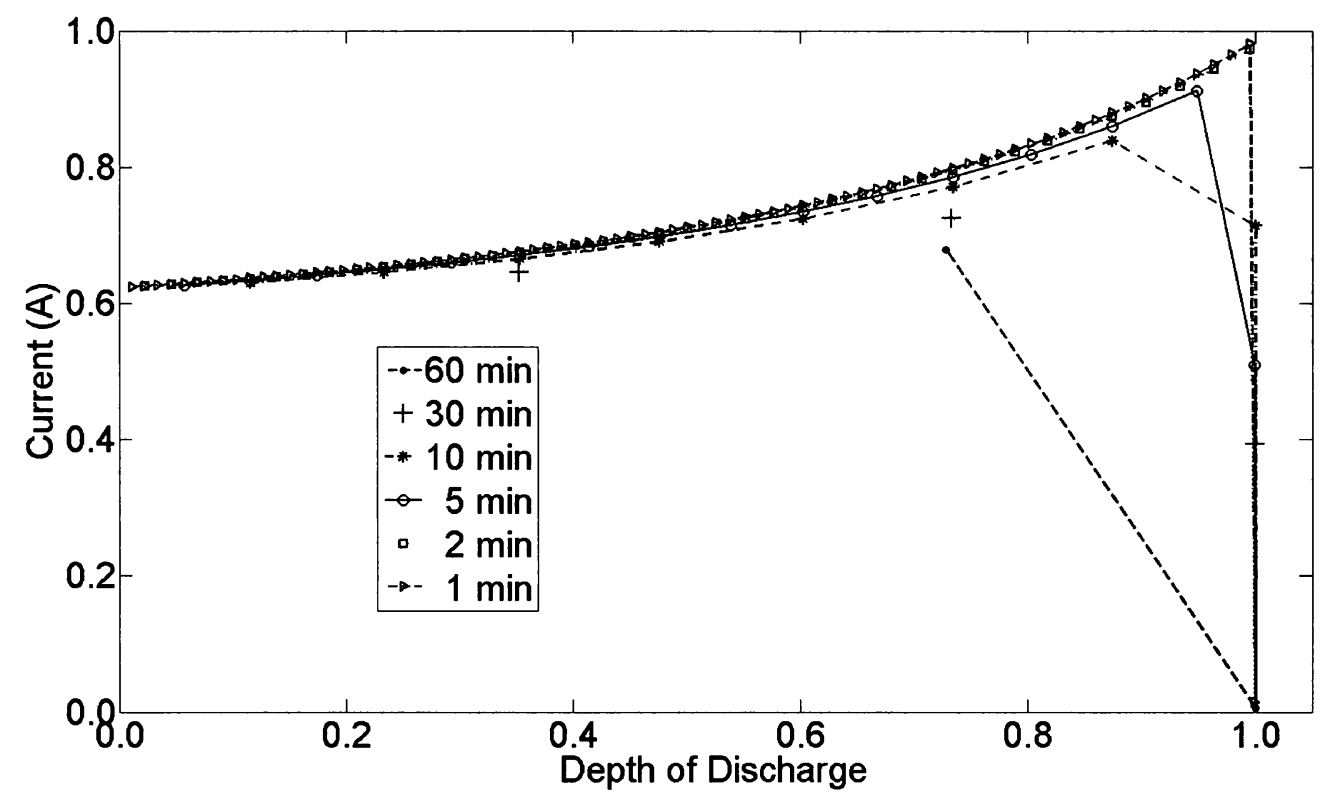

Figure 6.3: Comparison of same simulation in ESP-r with different time-steps to illustrate sensitivity.

As the battery approaches a DOD of one, the BMS reduces the current to zero, reducing the battery load to zero Watts to prevent over discharging. The time-step sensitivity is most apparent when the battery load changes. The 60 minute time-step simulation in figure 6.3 seems illogical because it has no current value below a DOD of around 0.75 . This is because its first current solved was after the first hour of the simulation, and by this time the cell had already discharged to this DOD. It appears that an acceptable time-step resolution would be between one and five minutes. If the battery load varies significantly, a time-step resolution closer to one minute may be necessary.

Returning to the initial comparison with ICPET, shown in figure 6.2 , the voltage curves compare reasonably as well. There is, however, a consistently lower voltage in the data from ICPET. The discrepancy of around $0.01 \mathrm{~V}$ can be attributed to a 
consistently higher current in the ICPET data, resulting in a higher internal resistance, and a larger voltage drop away from the OCV. With the model performing as expected, when compared with data from ICPET [48], it can now be integrated with a cogeneration device to simulate satisfying the electrical and thermal demands of a household.

\subsection{Demonstration of integrated system}

\subsubsection{ESP-r model description}

To demonstrate the capability of the li-ion model developed in this work, a model of a house was created in ESP-r for an annual simulation. The geometry of the house was basic: a single-zone, rectangular house located in Ottawa, ON. The model used typical constructions to represent the house's walls, ceiling and floor. It had a floor space of $132 \mathrm{~m}^{2}$ and a volume of $528 \mathrm{~m}^{3}$. These values were chosen to be close to those in a typical Canadian single family detached dwelling [2]. Because the objective here was to observe the functionality of a RES plant system using accurate household electricity usage data (elaborated further), emphasis was not placed on the detail of the house's geometry. A weather file of Ottawa, ON from 2008, collected from Environment Canada's Weather Office [5], was used for the simulation.

A schematic of the plant configuration used in the simulation is shown in figure 6.4. A SE model, developed by Ferguson [61] for IEA/ECBCS's Annex 42 [1], was used as the cogeneration device in the simulation. The SE was configured to constantly produce $900 \mathrm{~W}$ of electricity for the house throughout the year. The engine had a constant electrical efficiency of $9.29 \%$, and a constant thermal efficiency of $90.71 \%$. Some of the recovered heat was lost to the SE's surroundings as described by Ferguson [61]. For this example, the engine was placed outside of the house, exposed to ambient temperatures.

The useful heat recovered by the engine was circulated via a pump to an air-towater heat exchanger. The air-to-water heat exchanger, driven by a fan, supplied warm air to the household. The pump and the fan consumed $50 \mathrm{~W}$ and $25 \mathrm{~W}$ of electricity, each, in the simulation. Refer to figure 6.4 for this schematic.

The electric household demands were input as combined non-HVAC and space cooling demands on a five minute basis. One of the medium energy houses from the 


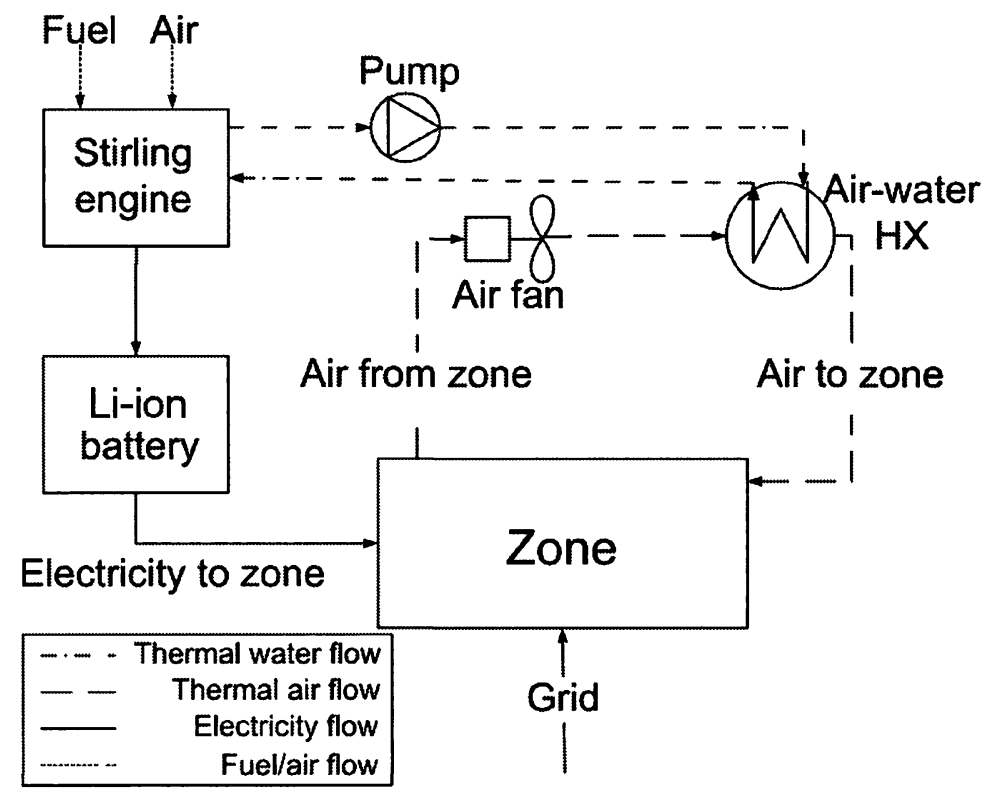

Figure 6.4: Plant system schematic for application demonstration.

data collection project, labelled $\mathrm{H} 6$ in section 3, was used for these demands. A SE in reality produces DC power and the battery stores DC power. A power conditioning unit (PCU) would traditionally transform this power to $\mathrm{AC}$ before sending it to the house. Because the data collected in the measurement project from this work (see section 3) recorded real power, i.e., DC, after taking into account the power factor, there is no need to condition the DC power to AC. Essentially, it is similar to having a PCU unit between the battery and the house with an assumed efficiency of $100 \%$. While this assumption may not be accurate in reality, it is sufficient to demonstrate this work's new modelling capabilities.

A control law needs to be implemented to dictate the function of each device in the plant system on how they will satisfy the household's energy demands. For this example, a simple control law was implemented to treat the house's electric and thermal demands. If the combined electricity demand from the household, pump and fan exceeded the $900 \mathrm{~W}$ produced by the $\mathrm{SE}$, the battery would discharge, within safe limits, to meet the excess. If the combined electricity demand from the household, pump and fan was less than the SE's production, the battery would charge with the excess electricity produced. If the SE and battery could not meet the combined electric demand, electricity would be imported from the grid. Likewise, if the SE's 


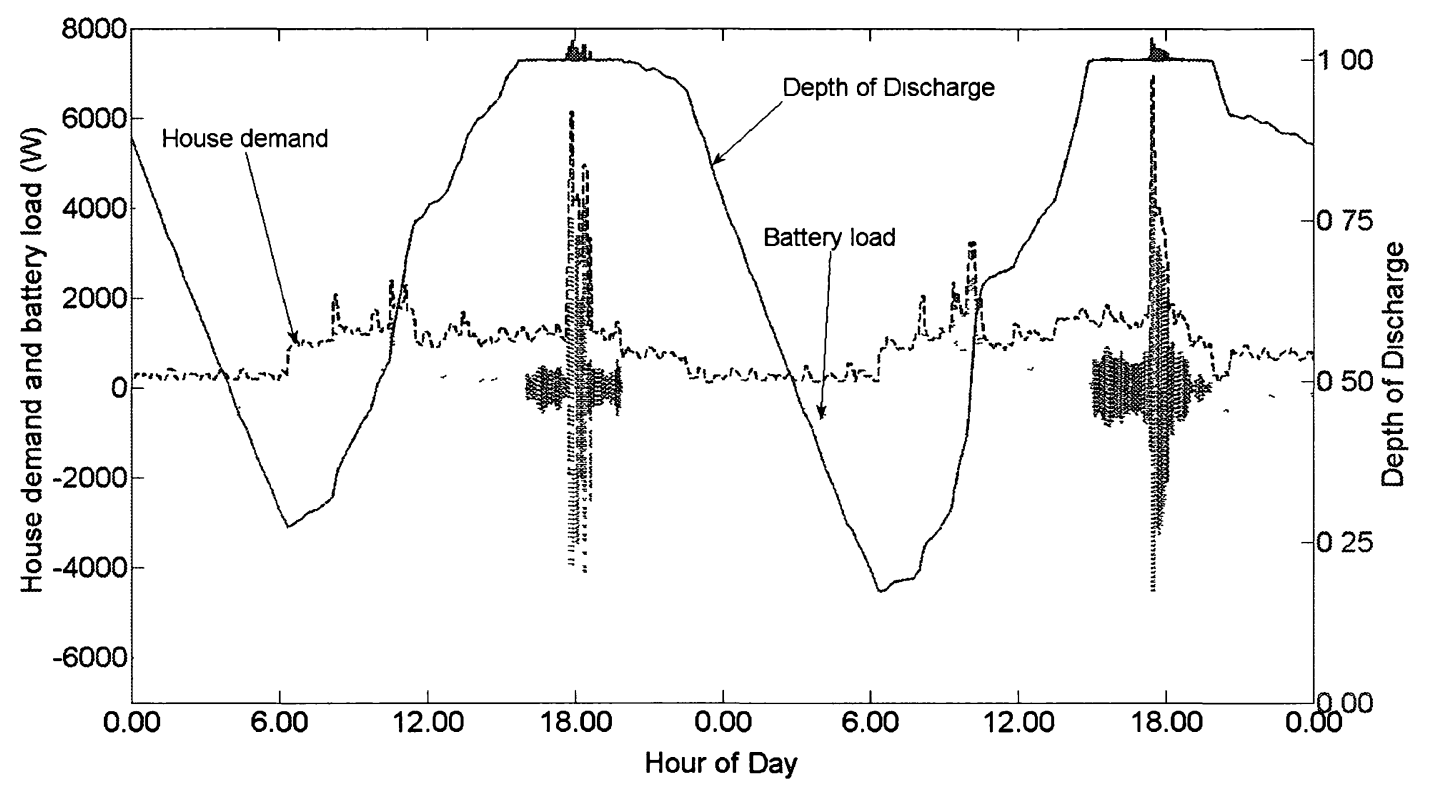

Figure 6.5: Household electricity demand, battery load and battery DOD over two days of simulation beginning August 4th, 2008. By convention, a positive battery load indicates a battery discharge and a negative indicates charging.

electricity production was greater than the combined electric demand and the battery was full, the excess electricity was exported to the grid.

The thermal demands were calculated by ESP-r to heat and maintain the dwelling at $20{ }^{\circ} \mathrm{C}$. No cooling setpoint was included in the simulation because all the space cooling loads were included in the electric loads. If the thermal demands of the household exceeded the heat produced by the engine via the heat exchanger, an auxiliary natural gas burner, not shown in the figure, would provide the necessary heat. If the thermal demands were less than the heat provided by the engine, the excess heat would be dumped to the ambient by venting the air outside.

\subsubsection{Simulation results}

Figures 6.5 and 6.6 show the battery operation from the simulation over a randomly selected two days in the summer, beginning on August 4th, 2008. Figure 6.5 shows the household's electricity demand, compared with the battery load and the battery's DOD. Figure 6.6 shows the battery's voltage, current and temperature over the same 
time frame.

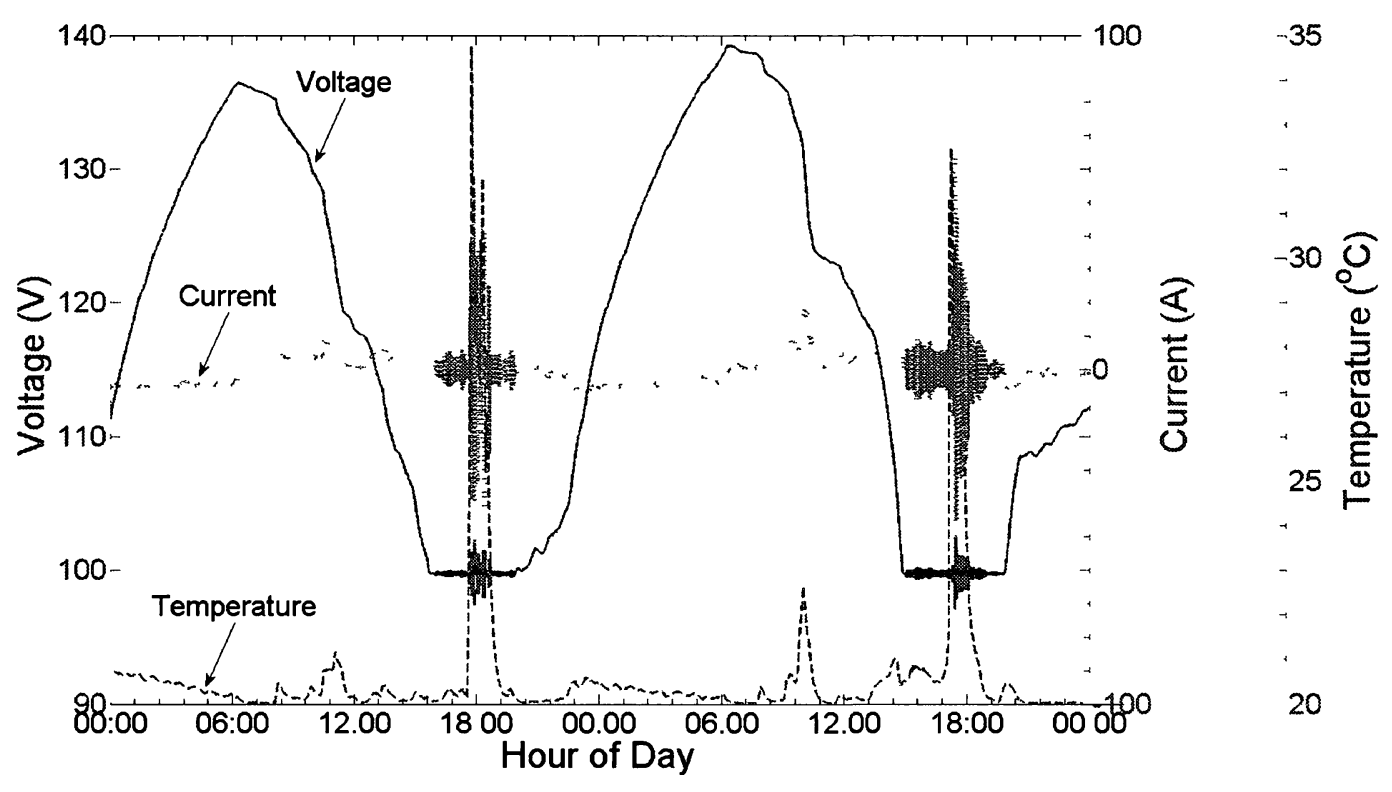

Figure 6.6: Battery voltage, current and temperature over two days of simulation beginning August 4th, 2008. By convention, a positive current indicates a battery discharge and a negative indicates charging.

By convention in these figures, a positive power and current value indicated that the battery was discharging while a negative value indicated charging. Over the nighttime of both days, the electric demand of the household was less than the production of the SE (see fig. 6.5). During these periods, the SE was able to satisfy the household demand and charge the battery with its excess electricity production. Over the daytime, the household electricity demand increased above the production of the SE and the battery began to discharge to satisfy it. Towards 18:00 on both days, the battery discharged too far while the demand was still too high. Here, electricity had to be imported from the grid to satisfy the demand. As the household activity dropped below the production of the SE in both evenings, the battery charged with the SE's excess electricity production.

The battery voltage, current and temperature seem to perform as expected (see fig. 6.6). As the battery discharged too far, it needed to charge itself back into a safe operating range. This is the reason for the rapid fluctuations in current at 18:00 on 


\begin{tabular}{cccc}
\hline & Discharge Current (A) & Charge Current (A) & Temperature ${ }^{\circ} C$ \\
\hline \hline Mean & 4.67 & 3.56 & 20.75 \\
\hline St. Dev & 7.79 & 3.75 & 1.80 \\
\hline Max. & 101.8 & 101.8 & 60.66 \\
\hline Min. & 0 & 0 & 20.00 \\
\hline
\end{tabular}

Table 6.1: Current and temperature statistics for annual simulation.

both days; the battery tried to meet the household demand, while the BMS tried to keep the battery in safe limits. This resulted in charging and discharging currents as high as $63 \mathrm{~A}$. The internal resistance resulting from the high current caused the battery temperature to rise from $20^{\circ} \mathrm{C}$ to $34.8^{\circ} \mathrm{C}$ (on the first day). The fluctuations of current at 18:00 on both days represents a flaw in the overall control strategy of the HVAC system. These current fluctuations would result in a loss of energy due to high internal resistances and would inflict damage on the battery. With a more sophisticated control on the HVAC system, these fluctuations could be avoided. This HVAC control strategy will be discussed in the future work section.

Table 6.1 lists information about the battery's charge current, discharge current and temperature over the entire year. It provides their means, standard deviations, maximum and minimum values. Using the battery degradation model developed in this work (see sect. 5.2.1), the battery used approximately 798 cycles over the year. This would equate to a lifespan of 3.76 years for a 3000 cycle battery life. If the long-term industry goal of a 10,000 cycle life span can be realized [62] this would equate to a lifetime of more than 12 years.

The values of table 6.1 show that the battery model behaves as expected over the entire simulation. Both discharge and charge currents have the same maximum value. These were for an empty battery or a full battery trying to charge or discharge at the maximum possible rate, respectively. The mean and standard deviation show higher values in discharge than in charge. This would seem appropriate because the charge was coming from a constant steady rate, when the house loads were low with no appliances. Whereas, the discharge was dictated by the highly varying household demand from appliances and space cooling. The battery temperature stayed close to the controlled temperature of the zone, shown by the mean and standard deviation. 
In periods of high charge and discharge, the temperature reached values greater than $60{ }^{\circ} \mathrm{C}$ before the BMS prevented further heating.

Another annual simulation was performed for comparison. This simulation was identical to the previous, except that the battery was removed from the plant system. Figures 6.7 and 6.8 shows the comparison of these two simulations.

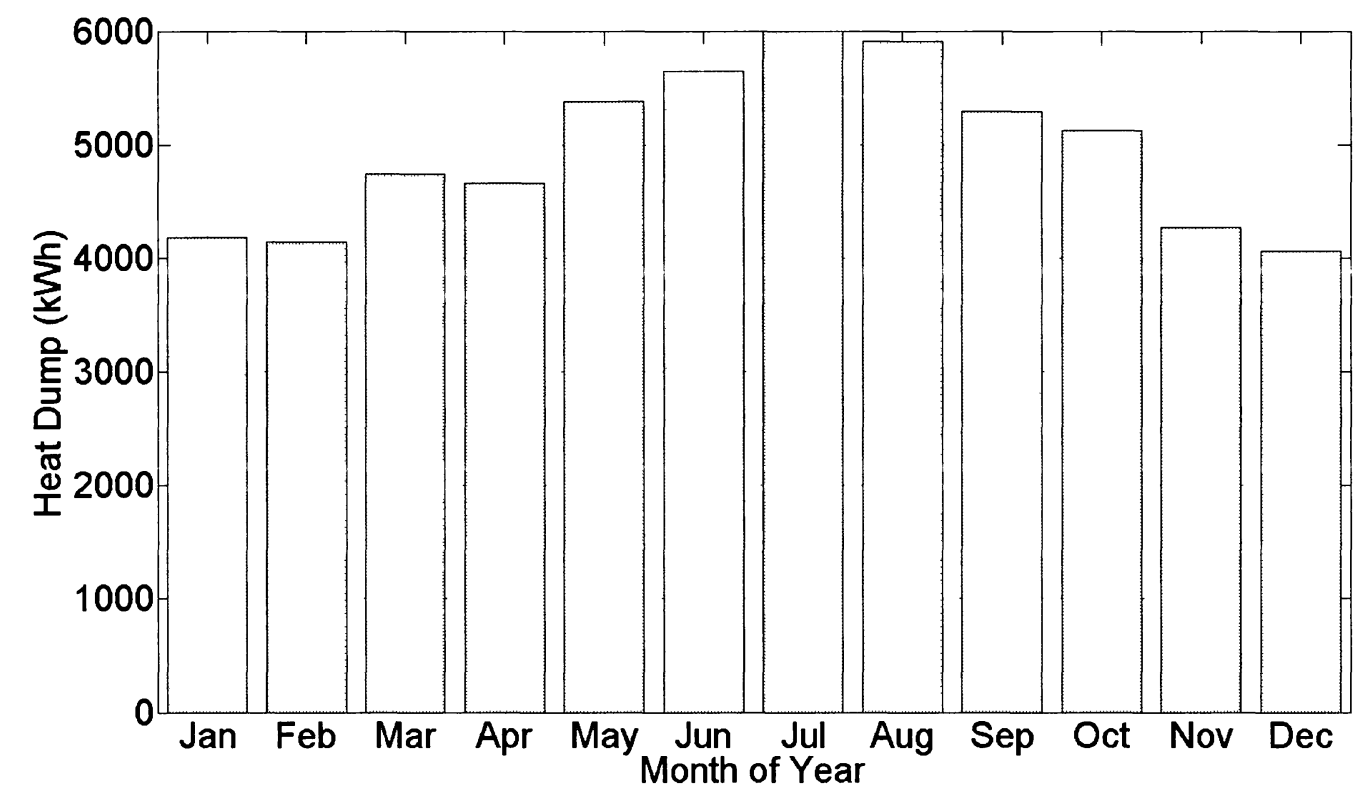

Figure 6.7: Total monthly heat dump from the SE to the ambient surroundings.

Figure 6.7 shows the monthly heat dumped by the SE, equal in both simulation scenarios. The heat generated by the SE was ample all year around to meet the thermal demands of the dwelling, and no auxiliary burner was required. This would indicate that this system was oversized in a thermal sense to meet the household demands. Note, however, that the thermal demands of the household were not the emphasis of this example. Proper treatment of infiltration with the exterior, windows and DHW considerations would change these results.

Figure 6.8 compares the total grid imports and exports of electricity on a monthly basis between the two scenarios. This figure shows that the coupling of a li-ion battery to a $\mathrm{SE}$ cogeneration device improved the performance of the system by reducing the grid interaction. In every month, the amount of electricity either imported from the 


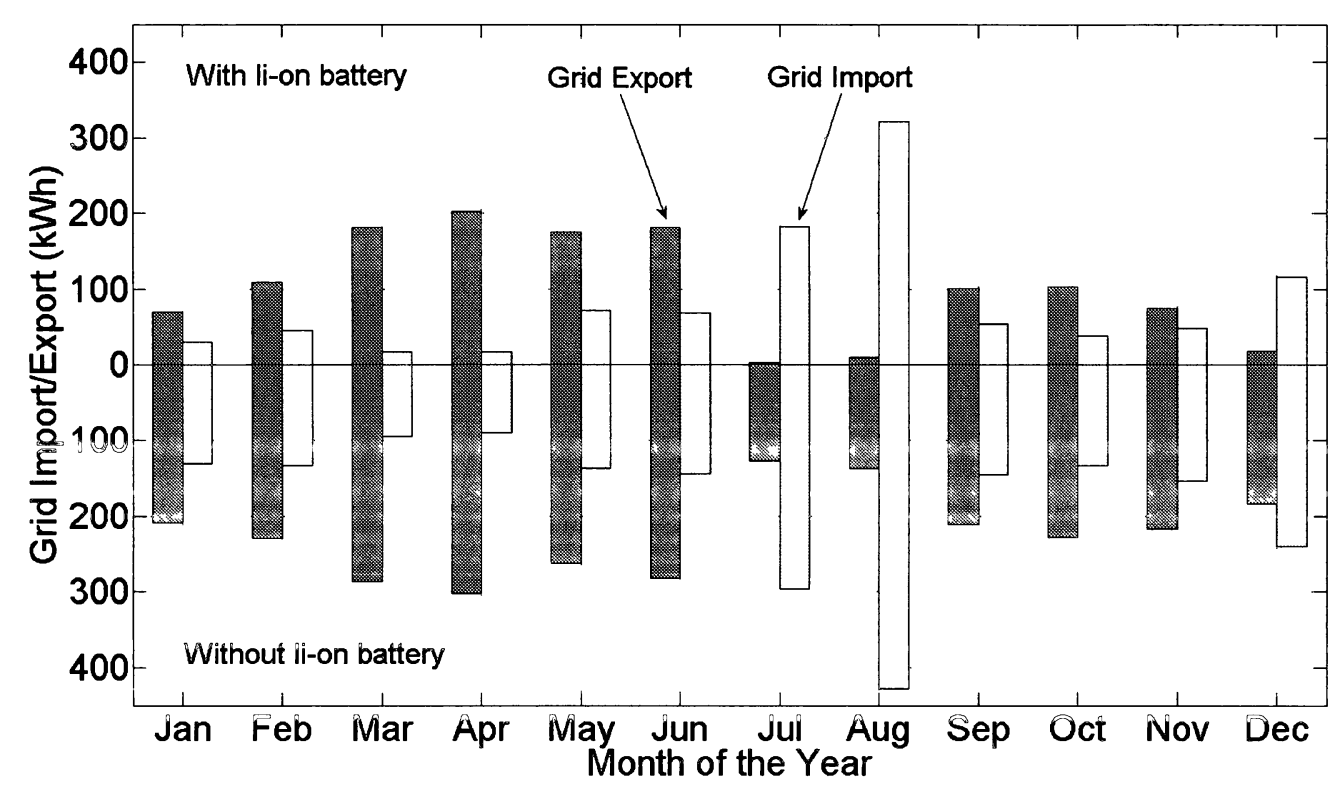

Figure 6.8: Simulation comparison with and without use of li-ion battery. The total monthly electric grid imports and exports are shown for both scenarios.

grid, or exported to the grid, was reduced. In general, the amount of electricity exported to the grid exceeded the amount of electricity imported. Once again this, indicates that the SE was oversized in this example.

The control law implemented in this example was quite simple; the battery acted essentially as a buffer between the electric demands of the household and the generation by the SE. More sophisticated controllers could significantly improve the performance of this system and reduce grid interaction further. The optimization of a custom controller developed for a RES system is complex, yet crucial to assess its full potential. The implementation of such a controller and a full assessment of a RES system are beyond the scope of this work. The future work section will elaborate on this further.

This chapter covered the testing and validation of the calibrated li-ion battery model developed in this work. It also demonstrated the new modelling capabilities of the li-ion model coupled with a SE using the residential electric usage data collected in this work. With this, an entire RES system model is ready to be optimally controlled and assessed to reduce household electricity consumption and peak demand. 


\section{Chapter 7}

\section{Conclusions \& Future Work}

\subsection{Conclusions}

Peak electricity demand in residential dwellings is a significant contributor of GHG emissions and inefficient electricity production. Preliminary assessments of microcogeneration devices, as a solution to peak power demand, recommended that electric storage may improve their ability to meet highly varying residential electric demand. Certain gaps existed to accurately assess a micro-cogeneration device coupled with battery storage using building simulation. Addressing these gaps were the objectives of this research.

One objective of this research was to gain a better understanding of residential electricity usage at a fine time resolution to improve the validity of building simulations. Non-HVAC and space cooling electricity usage of twelve homes in the Ottawa, Ontario area were gathered at one-minute intervals for a study period of one year beginning in the summer of 2009 .

The analysis of the non-HVAC data collected in the project showed that electricity usage varied significantly between households. The results showed that averaging data over a large number of days eliminated many of the peaks that occurred in the household, and was not a good method of analyzing peak power demand. Histograms, however, were able to show the distribution of electric draws, over many days, while still retaining the largest occurrences of electric draws. The analysis also showed, when compared to synthetically generated profiles, that residential electric loads may be too complex to capture in algorithms used for profile generation. A new technique, discussed in the future work section below, may offer a new approach in generating 
profiles. The data collected provides excellent simulation inputs for representing nonHVAC electricity use of Canadian households in building simulation.

The analysis of the space cooling data gathered in the project showed that airconditioning consumption varied between households. This was not only in total electricity consumed, but at what time of day as well. A scatter plot of the daily airconditioning electricity consumption with the maximum daily temperature and mean daily temperature showed a strong relationship that also varied between households. The data collected provides excellent validation of new algorithms in building simulation to capture the complexities of occupant habits with respect to space cooling; this is elaborated in the future work section below.

Another objective of this research was to develop a li-ion battery model and couple it with an existing cogeneration device in ESP-r. The model developed, based on an approach by Gao et al. [50], is able to account for variations in imposed current and battery temperature on performance. The model functionality includes long-term degradation, self-discharge, and thermal interaction with its surroundings, and can be sized to different capacities depending on its application.

Default calibration of the model was done with simulated data from NRC-ICPET [59] in chapter 5. The data provided was the cell voltage and DOD relationship of a simulated cell at different currents and temperatures. The model developed in this work was calibrated for a $6 \mathrm{~kW} \cdot h$ battery with a nominal voltage of $120 \mathrm{~V}$, using the data from ICPET and other data found in the literature review. Chapter 5 also explained the steps in calibrating different li-ion chemistries to the model. The model was validated with a different data set of a cell discharging at constant power, provided by ICPET, as well.

The new modelling capabilities, developed in this work, was demonstrated in Chapter 6. This was an annual simulation of the thermal and electric demands of a household being satisfied by a SE coupled with a li-ion battery. The electricity use data gathered in this work was used for the household's electric demands. While the example was quite simple in control, the implementation of electric storage saw the reduction of grid interaction with the house in every month. The model behaved as expected, consuming $27 \%$ of its useable life over the year, according to the battery degradation model implemented. The battery management system (BMS) kept the battery in safe charging and temperature ranges. The implementation of a custom optimized controller on the entire plant system would improve performance. This is 
discussed in the future work section below.

The objectives that were set out in this research have been successfully met. Recommendations for future work in this area is discussed below. With a full assessment of the potential of li-ion storage, coupled with micro-cogeneration, residential peak electricity demand and GHG emissions may be significantly reduced.

\subsection{Future Work: Non-HVAC and space cooling}

\section{Residential non-HVAC electricity future work}

Chapter 3 reviewed the electric measurement project, performed in this work, of Canadian houses. It showed the analysis of non-HVAC and space cooling electricity usage of twelve houses, in the Ottawa, ON area, at one minute intervals over one year.

As discussed in the literature review of non-HVAC electricity use (see section 3.1), similar projects aimed at producing randomly generated non-HVAC profiles, for use in building simulation, tend to use the data collected to develop algorithms. These algorithms are then used to produce new profiles. However, as seen in the results from this work on non-HVAC electricity use (see section 3.3), these algorithms generalize occupant activities into routines, and lack the complexity to truly represent the occupants' constantly changing lives.

To represent the stock of Canadian households with randomly generated profiles, a new technique discussed by Hill [63] may provide an interesting approach. This technique would use empirically measured data, similar to the work done in this research, to statistically analyze the distribution of electricity use. The analysis would be for not only means and variances, but also the correlations factors with respect to future time-steps, known as autocorrelation. An autocorrelation factor (ACF) reflects the relationship between a variable, $x$, at a present time-step, and the same variable $\mathrm{k}$ time-steps later. The value $\mathrm{k}$ is known as the lag.

$$
A C F=\frac{\sum_{\imath=1}^{n-k}\left(x_{\imath}-\bar{x}\right)\left(x_{\imath+k}-\bar{x}\right)}{\sum_{\imath=1}^{n}\left(x_{\imath}-\bar{x}\right)^{2}}
$$

An ACF of one, for a given time-step and lag $\mathrm{k}$, indicates a strong relationship between the variable at this time-step, and the variable at $k$ time-steps later. A value of negative one indicates a strong inverse relationship, and a value of zero indicates 
no relationship.

In the context of this work, the variable $x$ would be the non-HVAC electric draw of the household, at any time of the day. Histograms of household non-HVAC power draws, similar to the ones produces in section 3.3, represent the distribution of electric loads over the day. Autocorrelation would use this distribution and scatter the electric loads over the day according to relationship between them. New non-HVAC profiles, produced by random number generation, would be unique from the data collected, however, they would match in a statistical analysis sense. If more sub-metering of appliances could be performed, correlation between appliances, e.g., washer and dryer, oven and dishwasher, could be determined. If occupants were able to keep diaries of vacation, illnesses, family visits, holidays, etc., correlation could be made to these factors as well.

Figures 7.1 and 7.2 illustrate autocorrelation using a randomly selected day from one of the households in the project, divided into on-peak and off-peak times as defined in OPA's TOU pricing scheme [9], respectively. The ACFs in these figures were calculated up to a lag of 100 minutes after the present minute.

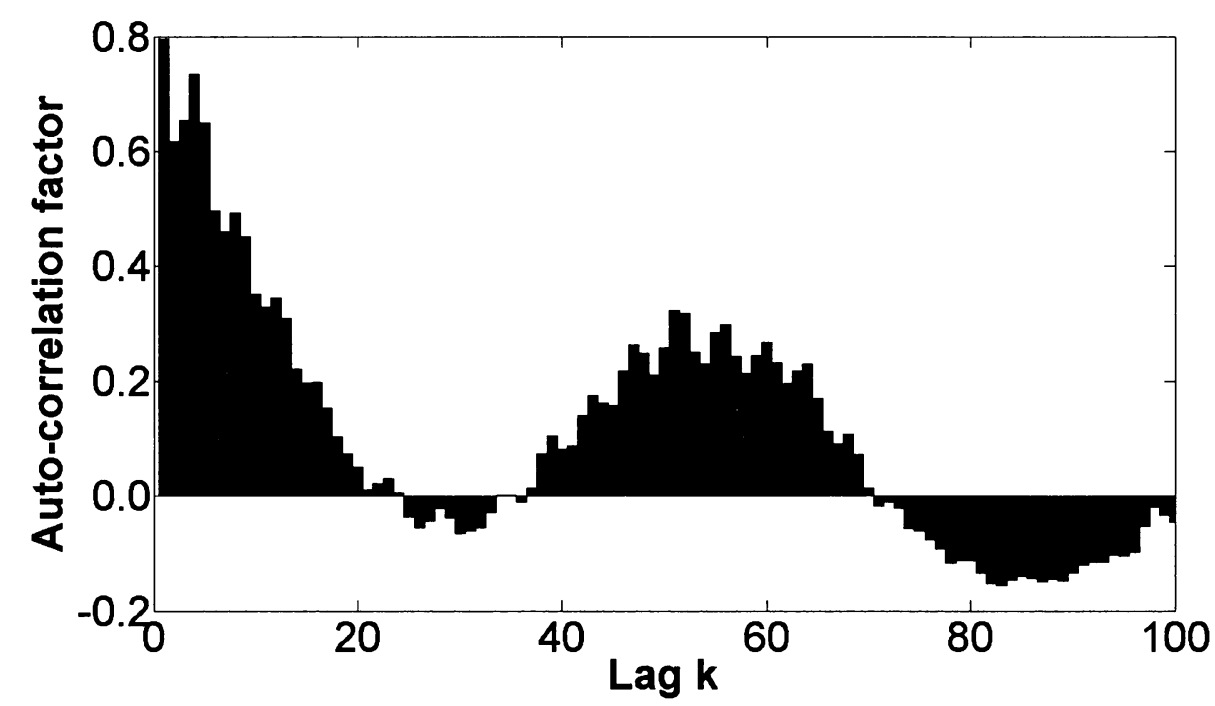

Figure 7.1: Auto-correlation as a function of lag $\mathrm{k}$ time-steps during on-peak hours for a randomly selected day of a randomly selected household in the project.

As the capital cost of purchasing monitoring equipment has already been incurred, more data can be continued to be collected into a database specific to the energy 


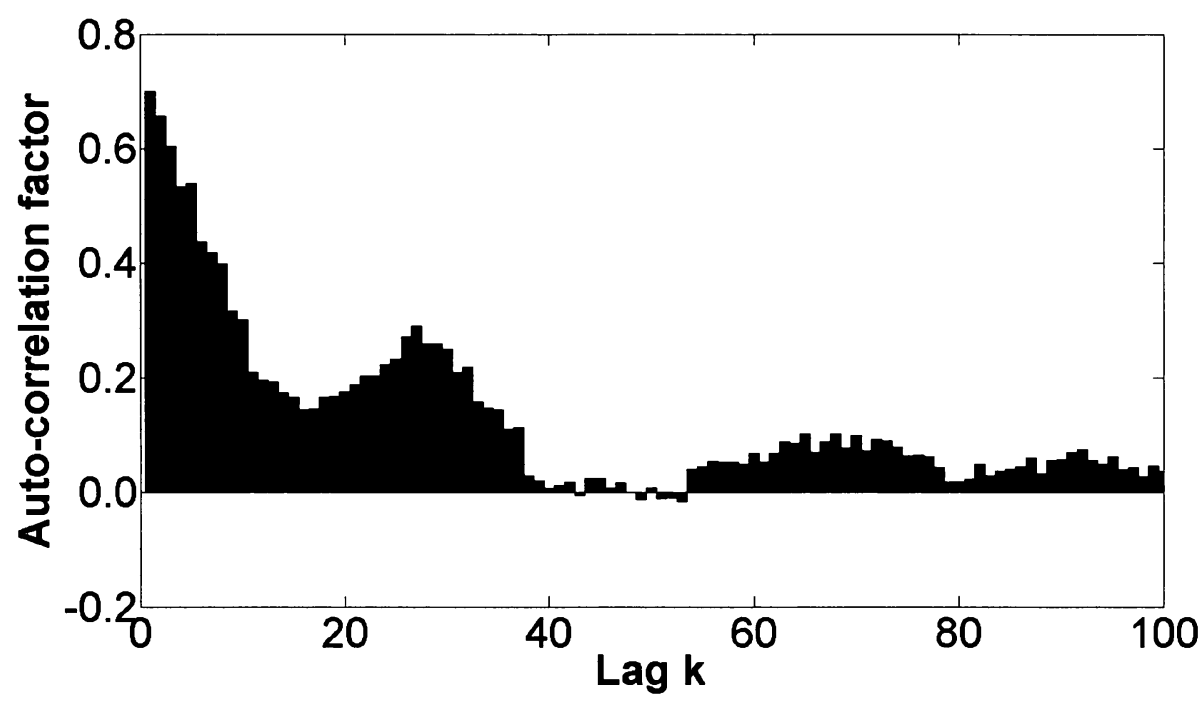

Figure 7.2: Auto-correlation as a function of lag $k$ timesteps during off-peak hours for a randomly selected day of a randomly selected household in the project.

consumption, location, size, number of occupants, etc. This database could be used to generate random statistically significant electricity use profiles of a specific household, if desired, or general profiles based on the entire database.

New strategies such as DSM, storage and micro-cogeneration could be simulated with the general database, with sensitivity analysis on specific houses, to observe the potential in specific cases. If the database could be randomly selected without bias, new strategy potentials could not only be assessed, but at what level of penetration they could occur. This could have residual effects on pricing schemes and costs of implementation.

\section{Residential space cooling electricity future work}

The space cooling data collected in this work provided excellent information on air-conditioning usage of Canadian houses. It was shown, in section 3.4, that airconditioning usage varied significantly between households. This can be attributed to several factors, as discussed, including window openings and shading.

A simulation of cooling loads, using only a temperature set-point to control airconditioning usage, would typically show different space cooling usage than the data collected in this work. This is because of occupant intervention in reality. BeausoleilMorrison's [30] work on implementing opening and shading algorithms in building 
simulation tries to realistically predict space cooling usage by simulating an occupant's intervention to mitigate it. With the information gathered in the survey that the occupants in the project filled out on openings, shading and their thermostat setpoints, new algorithms using random number generation can be implemented. These would based on the probabilities of occupant intervention with openings and shading. These algorithms could also incorporate more sophisticated thermostat control setpoints, in an effort to match the data collected in this project. New work in this area can use the space cooling usage data collected in this research for validation of these algorithms.

\subsection{Future Work: Li-ion battery model}

\section{Calibration of model with an actual cell}

The li-ion battery model developed in this work provides a novel method of incorporating an electric storage device in the building simulation tool ESP-r. The next step in this work is the calibration of the model with a commercially available li-ion cell. This would include charge and discharge curves of the cell at various constant currents and temperatures. This calibration would provide extensive validation in the modelling approaches used in this work, and provide new information of the complexities of real cells.

\section{Verification and improvements to the li-ion model}

Specific aspects of the li-ion model may need to be revisited, namely: the assumptions made in the heat transfer between the battery and its environment, battery degradation treatment, self-discharge parameters and parasitic power draws of the BMS. The realistic treatment of cell balancing in a battery would also need accurate treatment in the future. This could involve research into typical ways that cells become imbalanced in a battery pack. The implementation in the BMS of individual cell voltage monitoring and in the case of imbalances, discharging to keep the battery pack balanced, would approach a more realistic model. 


\section{RES control future work}

As discussed, the control of a plant system dictates the function of each component within, and is the most important factor in its performance. Simple controls of singlecomponent plant systems occur in a general way:

- Sense - A property is sensed in the model. This can be any property e.g., temperature, relative humidity, flow rates of plant systems and household energy demand.

- Control law - A control law is implemented to meet the sensed property. This can be as simple as an on/off switch control, or towards more sophisticated controllers such as proportional, differential, integrated (PID) controllers.

- Actuate - The device under the control law is actuated. This can be anything from the opening of valves, to increases of flow rates, to diversions of electricity flows like in the context of this work.

While these steps have been implemented in ESP-r and work well for single component systems, an integrated system with many components may require a custom sophisticated controller. These custom controllers are unique subroutines in the simulation program that dictate the function of all the components in the plant. Similar controllers have already been implemented in ESP-r [64].

A controller for RES should consider the energy sources and sinks of the model. In a thermal context, the sources are the cogeneration device and the sinks are the thermal demands of the house and DHW. In an electrical context, the sources are the cogeneration device and the sinks are the house's non-HVAC and space cooling electrical loads.

The storage device may act as a source or a sink. In an electrical context, this is the li-ion battery. Even in a thermal context, a hot water tank may be introduced for storage to improve performance.

A custom controller for RES could exist on many levels of resolution. The lowest level of controllers can involve the constant operation of the cogeneration device with the storage devices acting as buffers between the production and the demand. This is similar to the control implemented in the demonstration in section 6.3.

The next level of controller would involve either thermal load following or electric load following. In other words, the function of the cogeneration device would depend 
on either the household electric demand, or the household thermal demand. In this case, barring transient effects, the storage devices would be most effective at satisfying the demand of the load that is not being followed. In other words, a battery would be most effective in a thermal load following scenario, and a hot water storage tank would be most effective in an electric load following scenario.

Intermediate levels of controllers would involve assessing the state of energy of the storage devices for the operation of the cogeneration device. In other words, if the battery's SOC or the storage tank's temperature is too low, the cogeneration device would turn on. The cogeneration device would turn off once the battery's SOC or storage tank temperature reached a high value. This, however, still neglects transient warm up times of the cogeneration device. The control developed by BeausoleilMorrison et al. [64] works in this fashion.

High level controllers incorporate a broad range of definitions and can quickly become complicated. They can include consideration for transient operation, TOU pricing schemes, fuel and electricity costs, present household energy demand, and with the advantage of building simulation and weather forecasting, future household energy demand.

It is necessary to determine which parameter is being optimized in a high level controller. This could be GHG emissions, utility cost (to the user and/or to the utility provider), grid interaction minimization, or peak demand reduction, to name a few. These parameters are not necessarily mutually exclusive, and significant work should be done in this area to determine which parameter to maximize for system optimization.

Figure 7.3 is a brief, general, flow chart of a more sophisticated controller for a RES system. It attempts to reduce cost of electricity, specifically in peak demand periods. Because it follows an electric demand, adequate thermal storage would likely improve the thermal aspect of the system.

The sensing action compares the cost of producing electricity from the cogeneration device, compared to that of purchasing from the grid. This would consider the electrical efficiency of the device, and maybe the transmission losses from the grid. It could also consider the thermal demands of the house, and the cost of producing auxiliary heat. In this way, the device can include its thermal efficiency as an incentive to produce onsite. Another sensing option shown in figure 7.3 is to use the household's electric demand to govern the function of the system. This critical value can be the 
baseload, in an effort to reduce peak demand, or any demand, if the system is to meet all the household electric loads. The remainder of the control dictates the interaction between the power produced by the cogeneration device and the battery's SOC. The controller aims to use the battery as a buffer between the cogeneration device and the household in transient warm-up times, while the device's off-performance efficiency is poor.

This is just an example of the future work necessary in the control of a RES system. The next step, after an accurate assessment of a RES system being controlled in an optimized manner, would be the development of a battery prototype. This prototype would be based on the size and parameters recommended by the assessment. If feasible from the assessment, the prototype would provide the framework for the implementation of a li-ion battery coupled with a cogeneration device in residential households to reduce peak residential electricity demand. 


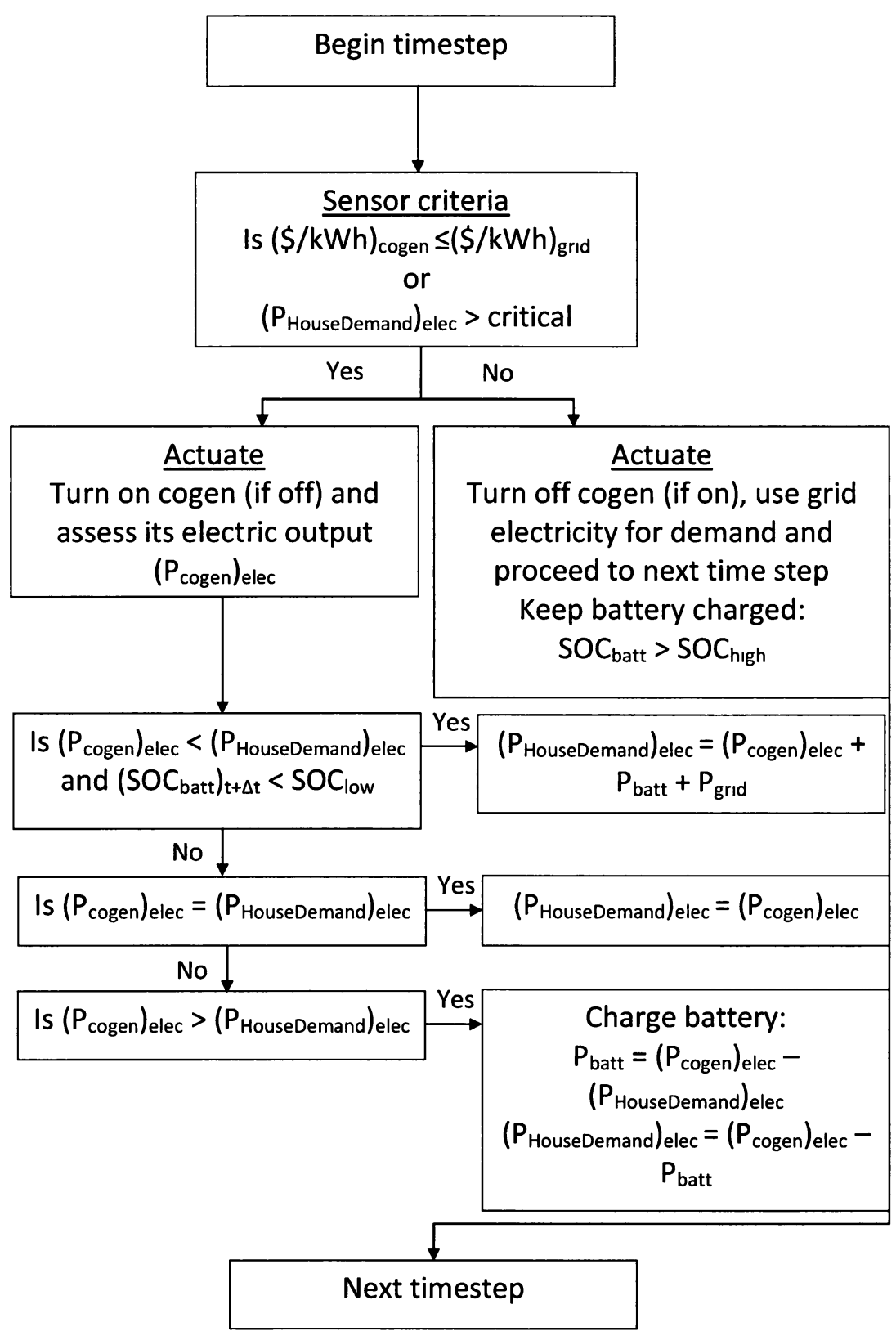

Figure 7.3: Example of custom controller flowchart for RES. 


\section{References}

[1] I. Beausoleil-Morrison, ed., An Experimental and Simulation-Based Investigation of the Performance of Small-Scale Fuel Cell and Combustion-Based Cogeneration Devices Serving Residential Buildings. IEA/ECBCS Annex 42 Report, 2008.

[2] "Energy use data handbook 1990 to 2007," tech. rep., Natural Resources Canada, 2009. Accessible online at http://oee.nrcan.gc.ca/publications/statistics/handbook08/index.cfm?attr $=0$.

[3] "Survey of household energy use 2003," tech. rep., Office of Energy Efficiency, Natural Resources Canada, 2005. Accessible online at http://oee.nrcan.gc.ca/Publications/statistics/sheu-summary/index.cfm.

[4] "Factor analysis of ontario electricity use - 1990-2003," tech. rep., ICF Consulting, Chief Conservation Officer-Ontario Power Authority, 2005.

[5] "Daily data report Ottawa, Ontario," tech. rep., Evironment Canada: Weather Office, 2010. Accessible online at http://climate.weatheroffice.gc.ca/.

[6] "Hourly generator output capability and demand for June 7th, 2008," tech. rep., Independent Electricity Service Operator, 2009. Accessible online at http://www.ieso.ca/imoweb/marketdata/genEnergy.asp.

[7] "Electric power generation, transmission and distribution," tech. rep., Statistics Canada, 2007. Accessible online at http://www.statcan.gc.ca/.

[8] "Ontario's integrated power system plan," tech. rep., Ontario Power Authority, 2007. Accessible online at http://www.powerauthority.on.ca/ipsp/.

[9] "Ontario energy board smart price pilot," tech. rep., IBM Global Business Services and eMeter Strategic Consulting for the Ontario Energy Board, 
2007. Accessible online at http://www.oeb.gov.on.ca/documents/cases/EB2004-0205/smartpricepilot/OSPP0Final

[10] R. Shaw, "The value of reducing distribution losses by domestic load-shifting: a network perspective," Energy Policy, vol. 37, no. 8, p. 3159, 2009.

[11] I. Knight and I. Ugursal, "Residential cogeneration systems: A review of the current technologies," tech. rep., Subtask A of FC+COGEN-SIM The Simulation of Building-Integrated Fuel Cell and Other Cogenearation Systems. Annex 42 of the International Energy Agency Energy Conservation in Buildings and Community Systems Programme, 2005.

[12] H. Ibrahim, "Energy storage systems - characteristics and comparisons," Renewable sustainable energy reviews, vol. 12, no. 5, pp. 1221-50, 2008.

[13] I. Hadjipaschalis, "Overview of current and future energy storage technologies for electric power applications," Renewable sustainable energy reviews, vol. 13, no. 6, p. 1513, 2009.

[14] G. Nazri and G. Pistoia, Lithium batteries: science and technology. Boston: Kluwer Academic Publishers, 2004.

[15] P. G. Bruce, "Energy storage beyond the horizon: Rechargeable lithium batteries," Solid State Ionics, vol. 179, no. 21, p. 752, 2008.

[16] J. Hassoun, "Recent advances in liquid and polymer lithium-ion batteries," Journal of materials chemistry, vol. 17, no. 35, p. 3668, 2007.

[17] G. Hunt, "The great battery search," IEEE Spectrum, vol. 35, no. 11, pp. 21 8, 1998.

[18] J. McDowall, Battery life considerations in energy storage applications and their effect on life cycle costing. Saft America at Power Engineering Society Summer Meeting, 2001.

[19] S. McGarry, "Lithium-ion battery life cycle." email correspondance, Department of Electronics, Carleton University, Ontario, Canada.

[20] D. Linden and T. B. Reddy, Linden's handbook of batteries. New York: McGrawHill, 2001. 
[21] H. Ibrahim, "Comparison and analysis of different energy storage techniques based on their performance index," 2007 IEEE Canada Electrical Power Conference (EPC '07), p. 393, 2007.

[22] I. Davidson, "Lithium ion market costs." email correspondance, Institute for Chemical Process and Environmental Technology, National Research Council Canada, 2009.

[23] "USABC goals for advanced batteries for EVs," tech. rep., United States Council for Automotive Research LLC, 2009.

[24] D. P. Jenkins, "Model for evaluating impact of battery storage on microgeneration systems in dwellings," Energy conversion and management, vol. 49, no. 8, p. 2413, 2008.

[25] Glasgow: University of Strathclyde, The ESP-r system for building energy simulations: user guide version 9 series, ESRU Manual U00/1, 2000.

[26] H. Ribberink and W. Wang, "Improving ESP-r's battery model with active battery life control and coverage of vanadium redox flow batteries," Proceedings eSim2008 Conference, 2008.

[27] J. A. Clarke, Energy Simulation in Building Design. Butterworth-Heinemann, 2nd ed., 2001.

[28] I. Knight, N. Kreutzer, M. Manning, M. Swinton, and H. Ribberink, "European and Canadian non-HVAC electric and dhw load profiles for use in simulating the performance of residential cogeneration systems," tech. rep., Subtask A of FC+COGEN-SIM The Simulation of Building-Integrated Fuel Cell and Other Cogenearation Systems. Annex 42 of the International Energy Agency Energy Conservation in Buildings and Community Systems Programme, 2007.

[29] M. Armstrong, M. Swinton, H. Ribberink, I. Beausoleil-Morrison, and J. Millete, "Synthetically derived profiles for representing occupant-driven electric loads in Canadian housing," Journal of Building Performance Simulation, vol. 2, no. 1, pp. 1-41, 2009. 
[30] I. Beausoleil-Morrison, "On predicting the magnitude and temporal variation of cooling loads in detached residential buildings," Building Simulation 2009 Conference Proceedings, 2009.

[31] P. A. Strachan, "History and development of validation with the ESP-r simulation program," Building and Environment, vol. 43, no. 4, p. 601, 2008.

[32] W. Wang, "Evaluation of the Alberta air infiltration model using measurements and inter-model comparisons," Building and Environment, vol. 44, no. 2, p. 309, 2009 .

[33] B. Lomanowski, "Implementation of window shading models into dynamic wholebuilding simulation," Master's thesis, University of Waterloo, Waterloo, Ontario, 2008 .

[34] N. Kelly, Towards a design environment for building integrated energy systems: The integration of electrical power flow modelling with building simulation. $\mathrm{PhD}$ thesis, University of Strathclyde, Glasgow, UK, 1998.

[35] J. A. Clarke, "Integrating power flow modelling with building simulation," Energy and Buildings, vol. 33, no. 4, p. 333, 2001.

[36] N. Isaacs, M. Camilleri, L. French, A. Pollard, K. Savill-Smith, R. Fraser, P. Rossouw, and J. Jowett, "Energy use in New Zealand households - HEEP year 10 report," tech. rep., BRANZ Group, New Zealand, 2006. Accessible online at http://www.branz.co.nz/.

[37] D. Parker, "Research highlights from a large scale residential monitoring study in a hot climate, FSEC-PF369-02," tech. rep., Florida Solar Energy Center, Cocoa, Florida, 2002. Accessible online at http://www.fsec.ucf.edu/en/publications/html/FSEC-PF-369-02/.

[38] G. Newsham, "Electrical load study of Ontario households." email correspondance, Institute for Research in Construction, National Research Council Canada, 2010.

[39] I. Beausoleil-Morrison, "A model for simulating the thermal and electrical production of small-scale solid-oxide fuel cell cogeneration systems within building simulation programs," HVAC R Research, vol. 12, no. 3, p. 641, 2006. 
[40] J. Widen, M. Lundh, I. Vassileva, E. Dahlquist, K. Ellegard, and E. Wackelgard, "Constructing load profiles for household electricity and hot water from timeuse data. Modelling approach and validation," Energy and Buildings, vol. 41, pp. 753-768, 072009.

[41] A. Capasso, W. Grattieri, R. Lamedica, and A. Prudenzi, "A bottom-up approach to residential load modeling," Power Systems, IEEE Transactions on, vol. 9, no. 2, pp. 957-964, 1994.

[42] J. V. Paatero and P. D. Lund, "A model for generating household electricity load profiles," International Journal of Energy Research, vol. 30, no. 5, pp. 273-290, 2006.

[43] C. F. Walker and J. L. Pokoski, "Residential load shape modelling based on customer behavior," IEEE Transactions on Power Apparatus and Systems, vol. PAS-104, pp. 1703-1711, 1985 JULY 1985.

[44] G. Gross and F. D. Galiana, "Short-term load forecasting," Proceedings of the IEEE, vol. 75, no. 12, pp. 1558-1573, 1986.

[45] H. K. Alfares and M. Nazeeruddin, "Electric load forecasting: literature survey and classification of methods," International Journal of Systems Science, vol. 33, pp. $23-34,2002$.

[46] R. Abernethey, R. Benedict, and R. Dowdell, "ASME measurement uncertainty," Journal of fluids engineering, vol. 107, pp. 161-164, 1985.

[47] R. Moffat, "Describing the uncertainties in experimental results," Experimental Thermal and Fluid Science, vol. 1, no. 1, pp. 3-17, 1988.

[48] K. Darcovich, "Residential electrical power storage scenario simulations with a large-scale lithium ion battery," Journal of Applied Electrochemistry, vol. 40, no. 4, pp. 749-755, 2010.

[49] R. Dell and D. A. J. Rand, Understanding batteries. Cambridge: Royal Society of Chemistry, 2001.

[50] L. Gao, S. Liu, and R. Dougal, "Dynamic lithium-ion battery model for system simulation," IEEE Transactions on Components and Packaging Technologies, vol. 25 , no. 3, pp. 495 - 505, 2002. 
[51] "Energizer technical bulletin: Battery internal resistance," tech. rep., Energizer Holdings, Inc., 2005. Accessible online at http://data.energizer.com/PDFs/BatteryIR.pdf.

[52] P. B. Balbuena and Y. Wang, Lithium-ion batteries : solid-electrolyte interphase. London: Imperial College Press, 2004.

[53] M. W. Verbrugge, "Electrochemical and thermal characterization of battery modules commensurate with electric vehicle integration," Journal of the Electrochemical Society, vol. 149, no. 1, p. A45, 2002.

[54] S. Soo, "Factors that affect cycle-life and possible degradation mechanisms of a Li-ion cell based on LiCoO2," Journal of Power Sources, vol. 111, no. 1, p. 130, 2002 .

[55] R. Spotnitz, "Simulation of capacity fade in lithium-ion batteries," Journal of Power Sources, vol. 113, no. 1, pp. 72-80, 2003.

[56] J. P. Holman, Heat tansfer. New York: McGraw-Hill Companies, 1997.

[57] P. Pinel, "Immosolars battery model: Battery and thermal management system," tech. rep., Immosolar ABT, Santa Ponsa, Spain, 2006.

[58] B. Y. Liaw, "Modeling of lithium ion cells - a simple equivalent-circuit model approach," Solid State Ionics, vol. 175, no. 1, pp. 835-9, 2004.

[59] K. Darcovich, E. Henquin, and I. Davidson, "Simulation of Li-ion battery discharge over a range of currents and operating temperatures," tech. rep., Natural Research Council of Canada Institute for Chemical Process and Environmental Technology, 2010.

[60] Maleki, "Thermal properties of lithium-ion battery and components," Journal of the Electrochemical Society, vol. 146, no. 3, p. 947, 1999.

[61] A. Ferguson, "Modelling residential-scale combustion-based cogeneration in building simulation," Journal of building performance simulation, vol. 2, no. 1, pp. 1-14, 2009. 
[62] K. Darcovich, "Lithium-ion long-term battery goals." email correspondance, Natural Research Council of Canada Institute for Chemical Process and Environmental Technology, 2010.

[63] J. Hill and B. Melamed, "TEStool: a visual interactive environment for modeling autocorrelated time series," Performance Evaluation, vol. 24, no. 1-2, pp. 3 - 22, 1995.

[64] I. Beausoleil-Morrison, M. Mottillo, A. Ferguson, H. Ribberink, L. Yang, and K. Haddad, "The simulation of a renewable-energy-powered hydrogen-based residential electricity system," Proceedings of simBuild 2006, 2006. 


\section{Appendix A}

\section{Example of calibration data provided from NRC-ICPET $\left(0.01 \mathrm{~A} \cdot \mathrm{m}^{-2}\right.$ and $\left.25^{\circ} \mathrm{C}\right)$}

\begin{tabular}{llll}
\hline Time $(\mathrm{s})$ & Cell Voltage $(\mathrm{V})$ & $L i_{\text {anode }}^{+}$ & $L i_{\text {cathode }}^{+}$ \\
\hline \hline 0.1 & 4.22266104 & 0.563469102 & 0.170603745 \\
\hline 2000 & 4.22253738 & 0.563098716 & 0.170687356 \\
\hline 4000 & 4.2222745 & 0.562739401 & 0.170769974 \\
\hline 6000 & 4.22201134 & 0.562380086 & 0.17085259 \\
\hline 8000 & 4.2217479 & 0.562020775 & 0.170935202 \\
\hline 10000 & 4.22148418 & 0.561661466 & 0.171017812 \\
\hline 12000 & 4.22122017 & 0.56130216 & 0.17110042 \\
\hline 14000 & 4.22095588 & 0.560942856 & 0.171183024 \\
\hline 16000 & 4.22069131 & 0.560583555 & 0.171265626 \\
\hline 18000 & 4.22042646 & 0.560224257 & 0.171348225 \\
\hline 20000 & 4.22016132 & 0.559864961 & 0.171430821 \\
\hline 22000 & 4.2198959 & 0.559505668 & 0.171513415 \\
\hline 24000 & 4.21963019 & 0.559146377 & 0.171596005 \\
\hline 26000 & 4.2193642 & 0.55878709 & 0.171678593 \\
\hline 28000 & 4.21909792 & 0.558427805 & 0.171761178 \\
\hline & & & \\
\hline
\end{tabular}


Appendix B

Calibration parameters for the Li-ion battery model 


\begin{tabular}{llll}
\hline ID & Symbol & Description & Units \\
\hline \hline 1 & nParal & number of cells connected in parrallel & $(-)$ \\
\hline 2 & nSeries & number of cells connected in series & $(-)$ \\
\hline 3 & cellCapRef & reference capacity for a unit cell (Amp h) & $(\mathrm{Amp} . h)$ \\
\hline 4 & tempRef & battery reference operating temperature & $\left({ }^{\circ} \mathrm{C}\right)$ \\
\hline 5 & maxVolt & DOD=0 of a single cell & $(\mathrm{V})$ \\
\hline 6 & minVolt & DOD=100 \% of a single cell & $(\mathrm{V})$ \\
\hline 7 & currentMax & maximum charge/discharge current of one cell & $(\mathrm{Amps})$ \\
\hline 8 & voltMax & maximum charge/discharge voltage of one cell & $(\mathrm{V})$ \\
\hline 9 & batTempInit & initial battery temperature & $(\%)$ \\
\hline 10 & batinitDOD & initial DOD & $(\%)$ \\
\hline 11 & thermalMass & thermal mass of a single cell m.Cp & $\left(\mathrm{J}^{\circ} \mathrm{C}^{-1}\right)$ \\
\hline 12 & heatLossFact & heat loss factor $\mathrm{h} * \mathrm{~A}$ & $\left(\mathrm{~W} \cdot \mathrm{C}^{-1}\right)$ \\
\hline 13 & heatersRes & resistance of the heaters & $(\Omega)$ \\
\hline 14 & upperBoundDOD & maximum DOD of battery & $(\%)$ \\
\hline 15 & lowerBoundDOD & minimum DOD of battery & $(\%)$ \\
\hline 16 & tempMax & maximum battery temperature & $\left({ }^{\circ} \mathrm{C}\right)$ \\
\hline 17 & tempMin & minimum battery temperature & $\left({ }^{\circ} \mathrm{C}\right)$ \\
\hline 18 & E5 & & \\
\hline 19 & E4 & $* * * * * * * * * * * * * *$ & \\
\hline 20 & E3 & OCV voltage as a function of DOD of a single cell & \\
\hline & & & \\
\hline
\end{tabular}




\begin{tabular}{|c|c|c|c|}
\hline ID & Symbol & Description & Units \\
\hline 21 & E2 & $V_{o c v}(D O D)=E 5 * D O D^{5}+E 4 * D O D^{4}+E 3 * D O D^{3}+E 2 * D O D^{2}+E 1 * D O D+E 0$ & \\
\hline 22 & E1 & $* * * * * * * * * * * * * * *$ & \\
\hline 23 & E0 & & \\
\hline 24 & Rint5 & & \\
\hline 25 & Rint4 & $* * * * * * * * * * * * * * *$ & \\
\hline 26 & Rint3 & Rint as a function of DOD at reference current of a single cell & \\
\hline 27 & Rint2 & $R_{\text {int }}(D O D)=\operatorname{Rint} 5 * D O D^{5}+\operatorname{Rint} 4 * D O D^{4}+$ & \\
\hline 28 & Rint1 & $\operatorname{Rint} 3 * D O D^{3}+\operatorname{Rint} 2 * D O D^{2}+\operatorname{Rint} 1 * \mathrm{DOD}+\operatorname{Rint} 0$ & \\
\hline 29 & Rint0 & $* * * * * * * * * * * * * * *$ & \\
\hline 30 & $\mathrm{c} 1$ & the first current discharged at & $(A m p s)$ \\
\hline 31 & $\alpha 1$ & the alpha required to discharge/charge at first rate & $(-)$ \\
\hline 32 & $\mathrm{c} 2$ & the second current discharged at & $(A m p s)$ \\
\hline 33 & $\alpha 2$ & the alpha required to discharge/charge at second rate & $(-)$ \\
\hline 34 & c3 & the third current discharged at & $(A m p s)$ \\
\hline 35 & $\alpha 3$ & the alpha required to discharge/charge at third rate & $(-)$ \\
\hline 36 & $\mathrm{c} 4$ & the fourth current discharged at & $(A m p s)$ \\
\hline 37 & $\alpha 4$ & the alpha required to discharge/charge at & $(-)$ \\
\hline 38 & $\mathrm{c} 5$ & the fifth current discharged at & $(A m p s)$ \\
\hline 39 & $\alpha 5$ & the alpha required to discharge/charge at fifth rate & $(-)$ \\
\hline 40 & c6 & the sixth current discharged at & $(A m p s)$ \\
\hline
\end{tabular}




\begin{tabular}{llll}
\hline ID & Symbol & Description & Units \\
\hline \hline 41 & $\alpha 6$ & the alpha required to discharge/charge at sixth rate & $(-)$ \\
\hline 42 & $\mathrm{c} 7$ & the seventh current discharged at & $($ Amps $)$ \\
\hline 43 & $\alpha 7$ & the alpha required to discharge/charge at seventh rate & $(-)$ \\
\hline 44 & $\mathrm{c} 8$ & the eighth current discharged at & $(\mathrm{Amps})$ \\
\hline 45 & $\alpha 8$ & the alpha required to discharge/charge at eighth rate & $(-)$ \\
\hline 46 & $\mathrm{~T} 1$ & the first temperature discharged/charged at & $\left({ }^{\circ} \mathrm{C}\right)$ \\
\hline 47 & $\beta 1$ & the beta required to discharge/charge at the first temperature & $(-)$ \\
\hline 48 & $\Delta V(T) 1$ & the voltage offset required to discharge/charge at the first temperature & $(\mathrm{V})$ \\
\hline 49 & $\mathrm{~T} 2$ & the second temperature discharged/charged at & $\left({ }^{\circ} \mathrm{C}\right)$ \\
\hline 50 & $\beta 2$ & the beta required to discharge/charge at the second temperature & $(-)$ \\
\hline 51 & $\Delta V(T) 2$ & the voltage offset required to discharge/charge at the second temperature & $(\mathrm{V})$ \\
\hline 52 & $\mathrm{~T} 3$ & the third temperature discharged/charged at & $\left({ }^{\circ} \mathrm{C}\right)$ \\
\hline 53 & $\beta 3$ & the beta required to discharge/charge at the third temperature & $(-)$ \\
\hline 54 & $\Delta V(T) 3$ & the voltage offset required to discharge/charge at the third temperature & $(\mathrm{V})$ \\
\hline 55 & $\mathrm{~T} 4$ & the fourth temperature discharged/charged at & $\left({ }^{\circ} \mathrm{C}\right)$ \\
\hline 56 & $\beta 4$ & the beta required to discharge/charge at the fourth temperature & $(-)$ \\
\hline 57 & $\Delta V(T) 4$ & the voltage offset required to discharge/charge at the fourth temperature & $(V)$ \\
\hline 58 & $\mathrm{~T} 5$ & the fifth temperature discharged/charged at in & $\left({ }^{\circ} \mathrm{C}\right)$ \\
\hline 59 & $\beta 5$ & the beta required to discharge/charge at the fifth temperature & $(-)$ \\
\hline 60 & $\Delta V(T) 5$ & the voltage offset required to discharge/charge at the fifth temperature & $(\mathrm{V})$ \\
\hline & & &
\end{tabular}




\begin{tabular}{|c|c|c|c|}
\hline ID & Symbol & Description & Units \\
\hline 61 & T6 & the sixth temperature discharged/charged at & $\left({ }^{\circ} \mathrm{C}\right)$ \\
\hline 62 & $\beta 6$ & the beta required to discharge/charge at the sixth temperature & $(-)$ \\
\hline 63 & $\Delta V(T) 6$ & the voltage offset required to discharge/charge at the sixth temperature & $(V)$ \\
\hline 64 & $\mathrm{~T} 7$ & the seventh temperature discharged/charged at & $\left({ }^{\circ} \mathrm{C}\right)$ \\
\hline 65 & $\beta 7$ & the beta required to discharge/charge at the seventh temperature & $(-)$ \\
\hline 66 & $\Delta V(T) 7$ & the voltage offset required to discharge/charge at the seventh temperature & $(V)$ \\
\hline 67 & $\mathrm{~T} 8$ & the eighth temperature discharged/charged at & $\left({ }^{\circ} \mathrm{C}\right)$ \\
\hline 68 & $\beta 8$ & the beta required to discharge/charge at the eighth temperature & $(-)$ \\
\hline 69 & $\Delta V(T) 8$ & the voltage offset required to discharge/charge at the eighth temperature & $(V)$ \\
\hline 70 & $k_{o}$ & self discharge constant & $(A)$ \\
\hline 71 & $E_{a s}$ & activation energy for self-discharge & $(k J) \cdot(k m o l)^{-1}$ \\
\hline 72 & iZoneIndex & the index of the zone where the battery is located & $(-)$ \\
\hline 73 & pParasitic & the power required to run the li-ion BMS circuitry & $(W)$ \\
\hline 74 & RintHi5 & $* * * * * * * * * * * * * * *$ & \\
\hline 75 & RintHi4 & SECOND OPTIONAL Rint as a function of DOD at reference current of a single cell & \\
\hline 76 & RintHi3 & $R_{\imath n t}(D O D)=\operatorname{Rint} 5 * D O D^{5}+\operatorname{Rint} 4 * D O D^{4}+\operatorname{Rint} 3 * D O D^{3}$ & \\
\hline 77 & RintHi2 & $+\operatorname{Rint} 2 * D O D^{2}+\operatorname{Rint} 1 * D O D+\operatorname{Rint} 0$ & \\
\hline 78 & RintHi1 & if any of these equal 999. they will be over riden and only the first set (24-29) will be used & \\
\hline 79 & RintHi0 & otherwise this will be used for I gt Iflag and (24-29) will be used for I lt Iflag & \\
\hline 80 & Iflag & Current value for if the current is higher the second set of Rint will be used & $(A)$ \\
\hline 81 & Cycle cruc $_{1}$ & The crucial cycle to indicate the end of the cell's life & \\
\hline
\end{tabular}

